\title{
Driving Point Impedance Measurements During Piano Playing
}

by

\section{Christianne Vant}

\author{
A Thesis submitted to \\ the Faculty of Graduate Studies and Research \\ in partial fulfilment of \\ the requirements for the degree of

\section{Master of Applied Science} \\ Ottawa-Carleton Institute for \\ Mechanical and Aerospace Engineering
}
Department of Mechanical and Aerospace Engineering Carleton University
Ottawa, Ontario, Canada

September 2007

Copyright (C)

2007 - Christianne Vant 


$\begin{array}{ll}\begin{array}{l}\text { Library and } \\ \text { Archives Canada }\end{array} & \begin{array}{l}\text { Bibliothèque et } \\ \text { Archives Canada }\end{array} \\ \begin{array}{l}\text { Published Heritage } \\ \text { Branch }\end{array} & \begin{array}{l}\text { Direction du } \\ \text { Patrimoine de l'édition }\end{array} \\ \begin{array}{l}\text { 395 Wellington Street } \\ \text { Ottawa ON K1A ON4 }\end{array} & \begin{array}{l}\text { 395, rue Wellington } \\ \text { Ottawa ON K1A ON4 } \\ \text { Canada }\end{array}\end{array}$

Your file Votre référence ISBN: 978-0-494-33672-4 Our file Notre référence ISBN: 978-0-494-33672-4

NOTICE:

The author has granted a nonexclusive license allowing Library and Archives Canada to reproduce, publish, archive, preserve, conserve, communicate to the public by telecommunication or on the Internet, loan, distribute and sell theses worldwide, for commercial or noncommercial purposes, in microform, paper, electronic and/or any other formats.

The author retains copyright ownership and moral rights in this thesis. Neither the thesis nor substantial extracts from it may be printed or otherwise reproduced without the author's permission.
AVIS:

L'auteur a accordé une licence non exclusive permettant à la Bibliothèque et Archives Canada de reproduire, publier, archiver, sauvegarder, conserver, transmettre au public par télécommunication ou par l'Internet, prêter, distribuer et vendre des thèses partout dans le monde, à des fins commerciales ou autres, sur support microforme, papier, électronique et/ou autres formats.

L'auteur conserve la propriété du droit d'auteur et des droits moraux qui protège cette thèse. $\mathrm{Ni}$ la thèse ni des extraits substantiels de celle-ci ne doivent être imprimés ou autrement reproduits sans son autorisation.
In compliance with the Canadian

Privacy Act some supporting forms may have been removed from this thesis.

While these forms may be included in the document page count, their removal does not represent any loss of content from the thesis.
Conformément à la loi canadienne sur la protection de la vie privée, quelques formulaires secondaires ont été enlevés de cette thèse.

Bien que ces formulaires aient inclus dans la pagination, il n'y aura aucun contenu manquant.

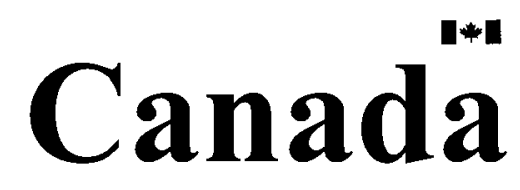




\section{Abstract}

Evidence indicates that $30-60 \%$ of professional piano players suffer from pain and playingrelated injuries at some point in their career. Most piano teachers emphasize the need for a relaxed wrist during playing to avoid these injuries.

Existing information regarding wrist actions that might trigger a stiffness change during playing is experiential. Many studies are conducted with a limited knowledge of either biomechanical principles or technical piano skills. The disciplines are integrated through a review of pedagogical approaches to piano technique, the clarification of terminology common to both disciplines and the application of biomechanical analyses to scientific data.

A review of pedagogical approaches showed that much of the information available regarding piano technique relies on metaphor to convey information from teacher to student on the feeling and actions required. Interpreting the biomechanical requirements of these techniques identified similarities and inconsistencies among them. Many imply specific biomechanical constraints on limb stiffness when describing a relaxed wrist.

Four specific concepts integral to the study of piano biomechanics are explained. Stiffness and co-contraction are represented as injurious in piano pedagogy, even though they are in fact necessary to the proper functioning of joint movements. Relaxation is touted as the solution to injury when in fact complete relaxation would not allow the limb to be held in the proper position for playing. Concepts are often approached from a single-joint point of view when in reality motions of the human body are interconnected. The change in position of a finger can affect all the joints in the arm. 
A new approach is developed to give an indication of changes in impedance at the wrist during piano playing. Experiments are conducted using a haptic device to apply force perturbations to the wrist during playing and to record the position of the wrist in threedimensional space. The displacement of the wrist due to the applied perturbations is quantified and analyzed as a measure of impedance at the wrist.

These experiments are unique because they are the first to regard changes in impedance at the wrist during piano playing and because the point of measurement is not at the endpoint but at a second point on the limb. The study is designed to create changes in impedance by increasing the dynamics (sound level), tempo and duration of playing. Subjects play music by Toronto composer Ann Southam written according to specifications of this study.

Statistical analysis of the data demonstrates the success of the force perturbations for the study of stiffness at the wrist. Data analysis was limited to one-dimensional motion in the vertical direction. The work demonstrates the practicality of measuring impedance during piano playing, however the music did not induce impedance changes in the two experienced piano players who underwent testing. 


\section{Acknowledgements}

There are many people who formed a part of my support network throughout my thesis work, both personally and professionally. To everyone I have dealt with for this project: thank you for the enthusiastic response - everyone has seemed not only interested but genuinely excited about the possibilities of this evolving research area. So many people are connected to musicians, or are musicians themselves, that there is a foreseeable impact resulting from the biomechanical analysis of music playing; this is research that everyone can relate to.

Thank you to my supervisor, Dr. Donald Russell, who supported me by trusting in my abilities and guiding me when I needed it; for always being helpful when I had questions, and for giving me the freedom to live life while in graduate school.

Thank you to everyone at the Piano Pedagogy Laboratory at the University of Ottawa, especially Gilles Comeau, Julia Brook and Jason Ray, who helped with the project since its inception and offered endless advice on how to make the project viable from the point of view of the piano community.

Thank you to Ann Southam for being excited about the chance to work with us and for composing music that adhered to our strict guidelines - I'm glad that you were up to the challenge.

Thank you to Dr. Ramesh Balasubramaniam for lending us your equipment and taking the time to introduce me to the haptic environment. 
Thank you to my friends in all the research labs around North America, but especially the ones here at Carleton: Martin, thanks for always offering help and advice; and Andrew, thanks for keeping the lab interesting during long hours spent at the computer and for helping me when I needed it.

And finally, thank you to my family, and especially my husband, for all your support throughout the years, your patience while I work my way through the rungs of academia and your enthusiasm for my achievements, big or small. And a special thanks to Shawn for letting me tap into some of your vast computer knowledge. 


\section{Table of Contents}

Abstract $\quad$ ii

Acknowledgements iv

Table of Contents vi vi

List of Tables $\quad$ ix

List of Figures $\quad$ x

1 Introduction $\quad 1$

1.1 Problem Statement . . . . . . . . . . . . . . . . . . 1

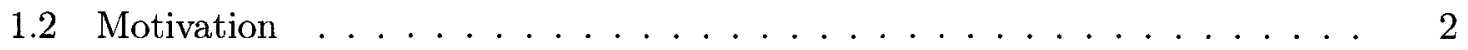

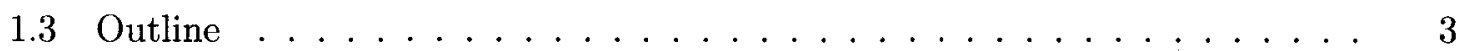

2 Biomechanics Background 5

2.1 Anatomical Orientation ........................ 5

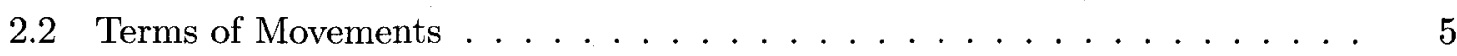

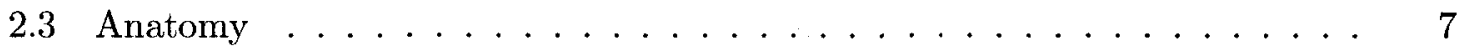

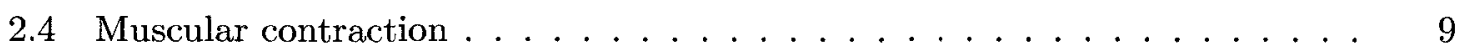

3 Literature Review 16

3.1 Historical Piano Biomechanics . . . . . . . . . . . . . . 16

3.2 Injury Analysis and Risk Factors f . . . . . . . . . . . . . . . . 18

3.3 Piano Technique . . . . . . . . . . . . . . . . . . 21

3.4 Evaluation of Piano Technique ................... 25

3.5 Stiffness Determination $\ldots \ldots \ldots \ldots \ldots \ldots$ 
4.1 Stiffness . . . . . . . . . . . . . . . . . 34

4.1.1 As a Measure of Modulus in Biomechanical Engineering . . . . . . . 34

4.1.2 Assessment of Several Uses in Pedagogical Literature . . . . . . . . . 40

4.2 Relaxation . . . . . . . . . . . . . . . . . . . . 43

4.2.1 As an Approximation for the Non-equivalent Terms of 'Stiffness' and 'Force' in Pedagogical Literature . . . . . . . . . . . . . . . . . . 44

4.2.2 As a State of Activation in Biomechanical Engineering . . . . . . . 45

4.3 Co-Contraction . . . . . . . . . . . . . . 50

4.3.1 A Common Misconception Presented in Pedagogical Literature . . . 50

4.3.2 Co-contraction in Biomechanical Engineering . . . . . . . . . 51

4.4 Multi-joint Issues $\ldots \ldots \ldots \ldots \ldots \ldots \ldots \ldots \ldots$

4.4.1 An Unrecognized Problem in Piano Pedagogy . . . . . . . . . . . . 53

4.4.2 Extension of Biomechanical Concepts From Single-Joint to Multi-Joint 54

5 Development of an Experimental Approach $\quad 58$

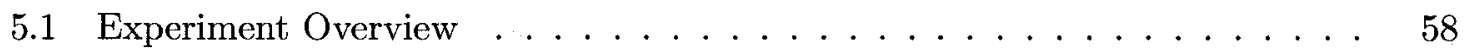

5.2 Apparatus . . . . . . . . . . . . . . . . . . 59

5.3 Methods ............................... 62

5.3 .1 Task Description . . . . . . . . . . . . . . . 62

5.3 .2 Experimental Software . . . . . . . . . . . . . . 64

5.3 .3 Perturbation Description $\ldots \ldots \ldots \ldots 67$

$\begin{array}{lll}6 & \text { Results } & \mathbf{7 0}\end{array}$

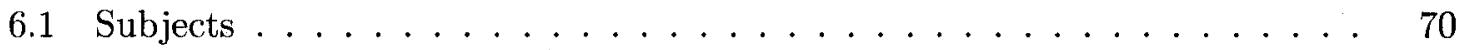

6.2 Polaris . . . . . . . . . . . . . . . . . . 71

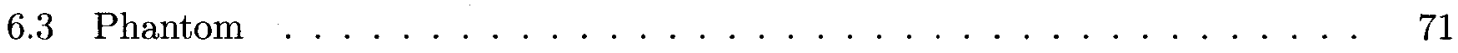

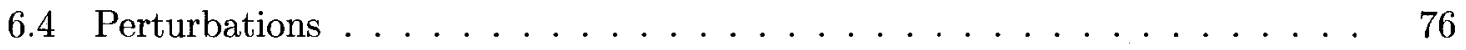

7 Data Interpretation $\quad \mathbf{8 5}$

7.1 Algorithms Based on Peak Heights . . . . . . . . . . . . . . . 86

vii 
7.1 .1 Pseudo Code . . . . . . . . . . . . . . 86

7.2 Algorithms Based on Time-Shifting Unperturbed Sections . . . . . . . . 87

7.2 .1 Data Preparation . . . . . . . . . . . . . . 88

7.2 .2 Pseudo Code . . . . . . . . . . . . . . . . 89

7.3 Algorithm Based on Symmetrical Attributes of Oscillatory Response . . . 90

7.3 .1 Pseudo Code . . . . . . . . . . . . . . . . . . 91

7.4 Perturbation Heights . . . . . . . . . . . . . . . . . . . . . 93

8 Discussion $\quad 101$

8.1 Outline of the Proposed Experimental Methodology . . . . . . . . . . . . 109

9 Conclusions and Recommendations $\quad 112$

9.1 Conclusions . . . . . . . . . . . . . . . . . 112

9.2 Recommendations . . . . . . . . . . . . . . . 113

$\begin{array}{ll}\text { List of References } & 117\end{array}$

$\begin{array}{ll}\text { Appendix A Creation of the Connector Block } & 125\end{array}$

$\begin{array}{lr}\text { Appendix B Music } & 128\end{array}$

Appendix C Code Listing 1: Software for the Experiment 131

Appendix D Mann-Whitney U Test $\quad 140$

Appendix E Code Listing 2-1: Basic Algortihm 150

Appendix F Code Listing 2-2: Time Shift Algortihm 154

Appendix G Code Listing 2-3: Symmetry Algortihm 158

viii 


\section{List of Tables}

5.1 Marker Definitions . . . . . . . . . . . . . . . 60

5.2 Summary of similar perturbation studies . . . . . . . . . . 67

7.1 Perturbation heights for subject $1 \ldots \ldots \ldots \ldots \ldots$

7.2 Perturbation heights for subject $2 \ldots \ldots \ldots \ldots$

8.1 Marker Definitions for the Proposed Methodology . . . . . . . . . . . . . 110

D.1 Statistical analysis for change in wrist height for subject 1, bars 1-4 . . . 142

D.2 Statistical analysis for change in wrist height for subject 2, bars 1-4 . . . 143

D.3 Statistical analysis for actual wrist height at 'note off' for subject 1, bars 1-4 144

D.4 Statistical analysis for actual wrist height at 'note off' for subject 2, bars 1-4 145

D.5 Statistical analysis for actual wrist height at 'note off' for subject 1, perturbation 5 , bars $5-8 \ldots \ldots \ldots \ldots \ldots \ldots \ldots$

D.6 Statistical analysis for actual wrist height at 'note off' for subject 1, perturbation 6 , bars $5-8 \ldots \ldots \ldots \ldots \ldots \ldots \ldots \ldots$

D.7 Statistical analysis for actual wrist height at 'note off' for subject 2, perturbation 5 , bars $5-8 \ldots \ldots \ldots \ldots \ldots \ldots \ldots \ldots \ldots$

D.8 Statistical analysis for actual wrist height at 'note off' for subject 2, perturbation 6 , bars $5-8 \ldots \ldots \ldots \ldots \ldots \ldots \ldots \ldots$ 


\section{List of Figures}

2.1 Anatomic planes ...................... 6

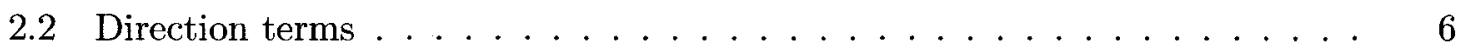

2.3 Common movements of the forearm $\ldots \ldots \ldots \ldots$

2.4 Anatomical names of the finger knuckles . . . . . . . . 8

2.5 Muscles of the forearm . . . . . . . . . . . . . . . . . 10

2.6 Muscles of the hand $\ldots \ldots \ldots \ldots \ldots \ldots$

2.7 Tetanic contraction . . . . . . . . . . . . . . 13

2.8 Muscular fatigue . . . . . . . . . . . . . . . . . . . . . . . 14

3.1 Curved finger position reduces the risk of injury . . . . . . . . . 20

3.2 Hand positions compared by Bejjani et al. (1989) . . . . . . . . . . . . 27

3.3 Manipulandum . . . . . . . . . . . . . . . . . . . 31

4.1 Passive Stiffness $\ldots \ldots \ldots \ldots \ldots \ldots \ldots$

4.2 Hill's Muscle Models . . . . . . . . . . . . . . . . . . . . . 37

4.3 Length-Tension Relationship _. . . . . . . . . . . . . . . 38

4.4 Isotonic contractions . . . . . . . . . . . . 47

4.5 Rest Length . . . . . . . . . . . . . . . . . . . . . . . . . 49

5.1 The Phantom Omni ....................... 60

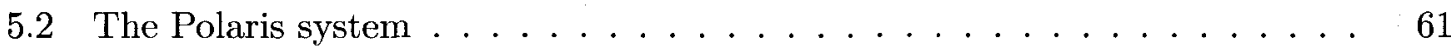

5.3 The markers on the subject while playing . . . . . . . . . . . 61

5.4 The Yamaha Disklavier grand piano . . . . . . . . . . . 62

5.5 The Phantom was attached to the subject using a block that was bandaged

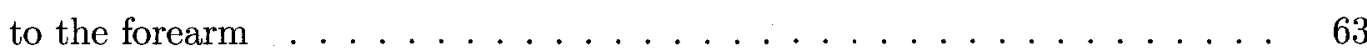


5.6 Free Body Diagram of Forearm and Hand . . . . . . . . . . . . . . 69

6.1 Three-dimensional position data of subject 1 measured by the Phantom . . 72

6.2 Three-dimensional position data of subject 2 measured by the Phantom . . 72

6.3 Plot of $\mathrm{x}, \mathrm{y}$, and $\mathrm{z}$ data with time for subject $1 \ldots \ldots \ldots$

6.4 Plot of $\mathrm{x}, \mathrm{y}$, and $\mathrm{z}$ data with time for subject $2 \ldots \ldots \ldots 74$

6.5 Three-dimensional position data of subject 1 measured by the Polaris . . . 75

6.6 Three-dimensional position data of subject 2 measured by the Polaris . . . 75

6.7 Displacement due to compression of soft tissue . . . . . . . . . . 76

6.8 Perturbations 1-4, Subject 1, Reading $1 \ldots \ldots \ldots \ldots 77$

6.9 Perturbations 1-4, Subject 1, Reading $2 \ldots \ldots \ldots$. . . . . . . 78

6.10 Perturbations 1-4, Subject 2, Reading $1 \ldots \ldots \ldots$. . . . . . 79

6.11 Perturbations 1-4, Subject 2, Reading $2 \ldots \ldots \ldots$

6.12 Perturbations $5-6$, Subject 1 , Reading $1 \ldots \ldots$. . . . . 80

6.13 Perturbations $5-6$, Subject 1 , Reading $2 \ldots \ldots \ldots$. . . . . . 81

6.14 Perturbations $5-6$, Subject 2, Reading $1 \ldots \ldots$. . . . . . 82

6.15 Perturbations $5-6$, Subject 2 , Reading $2 \ldots \ldots$. . . . . 83

7.1 Graphical version of the basic algorithms . . . . . . . . . . 86

7.2 Graphical version of the time shift algorithm $\ldots \ldots \ldots$. . . . . 88

7.3 Time-shifted music . . . . . . . . . . . . . . . 88

7.4 Graphical version of the symmetry algorithm . . . . . . . . . . . 91

7.5 Example of the basic onset algorithm functioning . . . . . . . . . 97

7.6 Example of the basic onset algorithm not functioning . . . . . . . . 97

7.7 Example of data normalization for subject $1 \ldots \ldots \ldots$

7.8 Example of data normalization for subject $2 \ldots \ldots \ldots \ldots$

7.9 Example of the symmetry algorithm functioning . . . . . . . . . 100

7.10 Example of the symmetry algorithm not functioning $\ldots \ldots \ldots$. . . . . 100

8.1 Comparison of perturbation heights between trials . . . . . . . . 107 


\section{Chapter 1}

\section{Introduction}

\subsection{Problem Statement}

Playing the piano is a complex task that requires both skill and training to do well. Although individuals devoted to piano performance can undergo many years of intensive tutelage and training, there is evidence of significant overuse injuries in 30-60\% of piano players [1]. This, and a lack of training in this area for piano teachers, leads to the inference that instruction is carried out with limited knowledge of the biomechanical impacts of the playing technique on the body. Thus the long-term effects of technique on the arm, and the wrist in particular, remain unknown.

The scientific study of piano playing is an interdisciplinary venture involving pianists, pedagogues, biomechanists, engineers and health professionals. The ultimate goal is to understand the biomechanical effects of various pedagogical approaches on performance, the biomechanics of piano playing, and the long-term effects.

One area of concern that is discussed in many pedagogical approaches is the topic of wrist stiffness (e.g. [2-5]) and the need to keep the forearm and wrist relaxed during playing. Information on the degree of stiffness change or actions that might trigger a stiffening wrist is anecdotal and experiential.

The objective is therefore to develop a method of measuring a variation in stiffness in the wrists of piano players. The corollary is to create a piece of music that generates a stiffening 
effect for this purpose.

The scope of this thesis is limited to monitoring variations in stiffness and to subsequently identifying any correlations between the variations in stiffness and the possible causes. The focus will be on establishing the degree of variation in stiffness and its variability between subjects and over time.

Limb stiffness analysis has almost universally employed the endpoint as the point of force measurement (e.g. [6-8]); mathematical models (e.g. [9]) are used to extrapolate the stiffness measured at the endpoint to joint stiffnesses. During the task of piano playing, the endpoint is otherwise engaged by depressing the piano keys and a new point of environmental interaction must be introduced. This point of force application is known as the driving point and for this work the driving point will be at the wrist.

The proposed experiments are novel because they are performed during piano playing and also because stiffness will be measured at a second point of interaction, or driving point - the wrist.

\subsection{Motivation}

Overuse syndrome, similar to what was originally known as "pianist's breakdown", has been recognized since the 19th century. It has been described as a painful condition brought about by long, hard use of a limb that is excessive for the individual affected, taking the tissues beyond their biological tolerance and causing some subsequent change $[10,11]$ and as a condition caused by tissues being stressed beyond their anatomic and physiological limits [12-14].

The condition has been reported in up to $50 \%$ of professional orchestra musicians [15] and $21 \%$ of music students [16]. Bejjani et al. [17] state that the only effective treatment since it was first observed is rest from the activities that cause pain and aggravate the condition. 
A study of young pianists found that overuse syndrome of the elbow, wrist or hand was prevalent in 28 of 66 players (42\%) [18]. Another study found $65.1 \%$ of the 341 respondents presented playing-related repetitive strain injury (RSI) at some point during their life [19], while another found a playing-related injury rate of $25.8 \%$ [20]. In this last case injuries were defined as preventing playing for a period of 48 hours or more.

A Philadelphia-area study of piano majors found that $42 \%$ of respondents experienced discomfort which lasted for more than one week. The playing of $87 \%$ of these was modified as a result; they altered their practice habits $(38 \%)$, altered their technique $(23 \%)$, ceased practicing $(21 \%)$ or changed repertoire $(11 \%)$. [21]

A 14-year study of music performance majors tallied 513 cases of performance-related upper-extremity injury. The study found an overall injury rate of 8.3 (per 100 performance majors), but piano was found to be a high-injury-rate instrument with an injury rate of between 12.0 and 18.0 [22]. According to Brandfonbrener et al. [23], keyboard and string instruments are often found to have a higher degree of risk than other instruments.

It is difficult to analyze the economic impact of these injuries since piano players are often not paid if they do not play. The true cost includes loss of income as well as medical costs, which can include surgery, physiotherapy and various other specialties. In 1989, Liberty Mutual Insurance Company received 6,067 worker's compensation claims for upper extremity cumulative trauma disorders throughout 45 states. They paid an average of $\$ 8070$ USD per case, with a median cost of $\$ 824$ USD. These figures represent both medical costs $(32.9 \%)$ and indemnity costs $(65.1 \%)$. The total cost for these disorders in the United States during the study year was estimated at $\$ 563$ million. [24]

\subsection{Outline}

The thesis is divided into three main sections. The first is the introduction and explanation of important concepts and issues. This is presented in Chapters 1 through 4. Chapter 
1 presents the problem statement and the motivation for pursuing the problem. Chapter 2 presents an explanation of relevant biomechanical terminology and concepts. Chapter 3 gives an overview of the relevant research completed in the both fields of piano pedagogy and biomechanics. Chapter 4 clarifies the meanings and usage of existing terminology in an attempt to merge the language of the two fields.

The next section focuses on the application and extension of existing knowledge through the development and validation of a methodology for measuring stiffness at the wrist during piano playing. This is presented in chapters 5 through 8 . Chapter 5 develops testing methods for biomechanical experiments during piano playing. The technique is validated in chapters 6 and 7. The results of the biomechanical experiment are presented in chapter 6 and chapter 7 analyzes these results using a variety of interpretations. Finally, chapter 8 discusses the findings in a broader context.

The final section gives the direction for future work in this interdisciplinary research. Chapter 9 summarizes the findings and conclusions of this study as well as recommends some next steps to take. 


\section{Chapter 2}

\section{Biomechanics Background}

Biomechanics is defined as the study of the mechanical laws relating to the movement or structure of living organisms [25]. The movement and structure of the human body is well-defined, so it is important to describe certain terminology prior to reviewing the biomechanical literature.

\subsection{Anatomical Orientation}

The orientation of the body uses a coordinate system composed of the three planes shown in Figure 2.1: sagittal, frontal and transverse. The sagittal plane divides the body longitudinally into left and right parts. If it were to divide into left and right halves, this would be called the median plane. The frontal plane also divides the body longitudinally, but into front and back parts. The transverse plane divides the body into upper and lower parts.

There are many terms that can be used for direction, but only two are required here (Figure 2.2). Proximal is a direction that is only used in reference to limbs; it means towards the body, or median plane. For instance, the shoulder is proximal to the hand. The opposite of this is called distal.

\subsection{Terms of Movements}

The bending and rotation actions of each joint have specific terminology dependent on the direction of motion, see Figure 2.3. Adduction is a motion where a joint moves a bone 


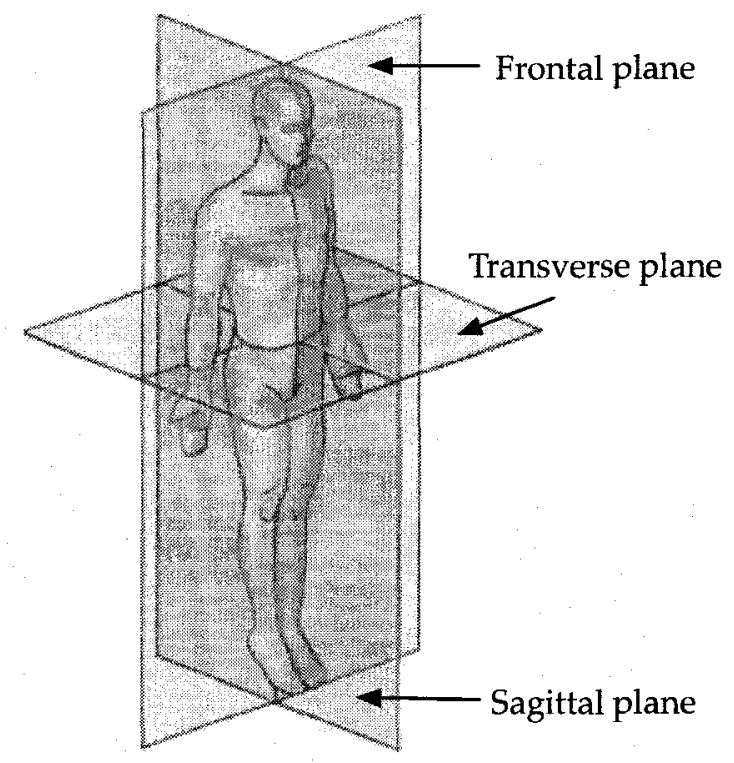

Figure 2.1: The anatomic planes for orientation to the human body are frontal, transverse and sagittal. [Figure adapted from [26]]

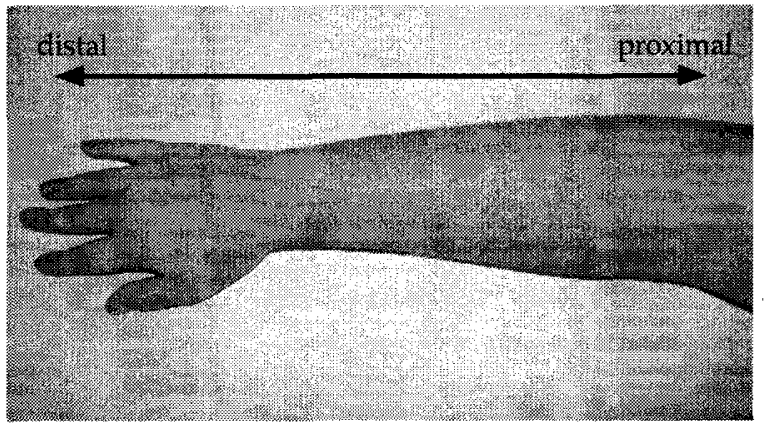

Figure 2.2: Use the terms proximal, or closer to the body, and distal, or further from the body, when referring to a limb. 
towards the midline of the body, hand or foot. The opposite movement, abduction moves the bone away from the midline. Pronation is the internal rotation of the radiohumeral joint (part of the elbow). The motion is a rotation of the forearm and hand in the direction that brings the thumb towards the middle. A rotation of the radiohumeral joint in the opposite direction is called supination. Flexion is the bending of a joint, which decreases the angle between the bones of the joint. The opposite of this movement is extension, or the straightening of a joint.

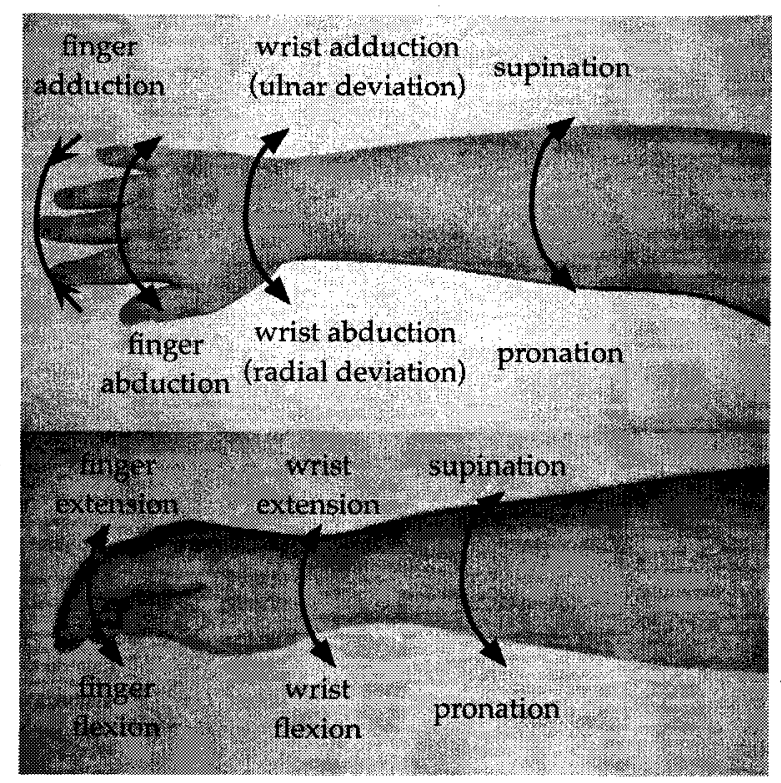

Figure 2.3: Pronation and supination are rotations from the elbow. Pronation is an internal rotation and supination is an external rotation. Adduction and abduction are movements of a bone towards and away from the midline of the body, respectively. Flexion is to bend a joint and extension is to straighten it.

\subsection{Anatomy}

A basic overview of the anatomy of the upper extremity is presented. Joints are the locations that connect bones and allow movements to occur. They are composed of connective tissues and synovial cavities, which are essentially fluid-filled sacs. The joints of the upper extremity are listed below starting at the proximal end, or the end nearest the body. The knuckles are shown in Figure 2.4; the metacarpophalangeal joint (MCP) is denoted by ' $\mathrm{a}$ ', the proximal interphalangeal joint (PIP) by ' $b$ ' and the distal interphalangeal joint (DIP) 
by 'c'.

- The shoulder, a ball-and-socket joint joining the clavicle to the humerus, or upper arm;

- The elbow, a hinge joint joining the humerus to the ulna and radius, which are the bones of the forearm;

- The wrist, an ellipsoid joint joining the ulna and radius to the many carpal bones that compose the palm of the hand;

- The carpometacarpal joint (CMC), or the base of the thumb, a saddle joint joining the carpal bones of the hand to the first metacarpal bone of the thumb;

- The metacarpophalangeal joint (MCP), or first knuckle, an ellipsoid joint joining the carpal bones to the proximal phalanx, or first finger segment;

- The proximal interphalangeal joint (PIP), or second knuckle, a hinge joint joining the proximal phalanx to the middle phalanx, or second finger segment; and

- The distal interphalangeal joint (DIP), or third knuckle, a hinge joint joining the middle phalanx to the distal phalanx, or end finger segment.

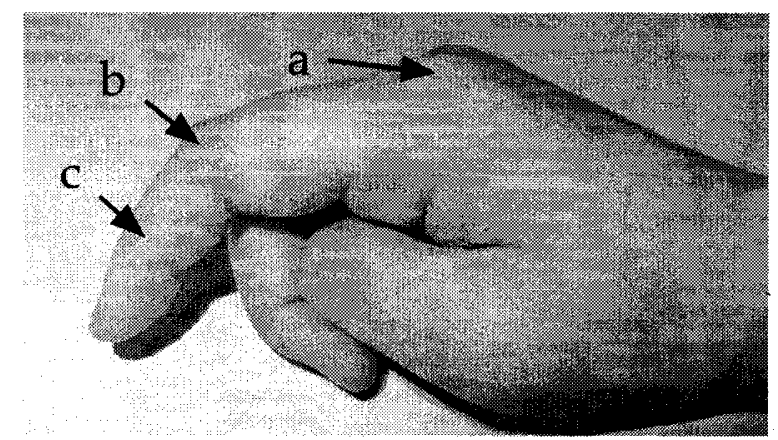

Figure 2.4: The anatomical names of the finger knuckles from the proximal end are: (a) metacarpophalangeal joint, or MCP (b) proximal interphalangeal joint, or PIP and (c) distal interphalangeal joint, or DIP.

Note that the thumb has no middle phalanx, and so it only has one interphalangeal joint which joins the proximal phalanx to the distal phalanx. 
Skeletal muscles are an active component of human anatomy. They are responsible for moving joints and hence the skeleton. Muscles attach to bone via connective tissue called tendons. The tension in a muscle increases when it is active and since it pulls on bone via the tendon, the tendon tension also increases. In the research presented here, the tension in the muscle and in the tendon are assumed to be the equal. Some of the important muscles for this overview are described below.

Flexor digitorum profundus (FDP) is a deep muscle on the forearm and flexor digitorum superficialis (FDS) is an intermediate layer muscle on the forearm. They are both responsible for flexing the fingers at the MCP joint. The flexor carpi ulnaris, palmaris longus and flexor carpi radialis muscles are superficial layer muscles on the forearm. Their primary responsibility is to flex the wrist. The interosseus muscles (INT), palmar interosseus and dorsal interosseus, are deep muscles of the hand that occur on both the palmar side and the dorsal side (back) of the hand. Their main function is adduction and abduction of the fingers. Muscles are involved in more than one movement, only their primary purpose is listed here. Muscles are shown in Figures 2.5 and 2.6.

\subsection{Muscular contraction}

Skeletal muscle is an active tissue that is infiltrated with nerve endings for communication with the central nervous system - either through reflexes or voluntary control. These muscles are composed of structural units known as muscle fibres, which are bundled parallel to each other. Each fibre is in turn composed of myofibrils, which are the basic unit of contraction in a muscle. The myofibrils are striated in repeating patterns known as sarcomeres. The striations are due to the arrangement of the different proteins that make up the myofibrils. The contractile proteins in the myofibrils are actin and myosin, the thin and thick filaments respectively. The contraction of the muscle is due to the interaction of these two proteins, which is described by the sliding filament theory. 


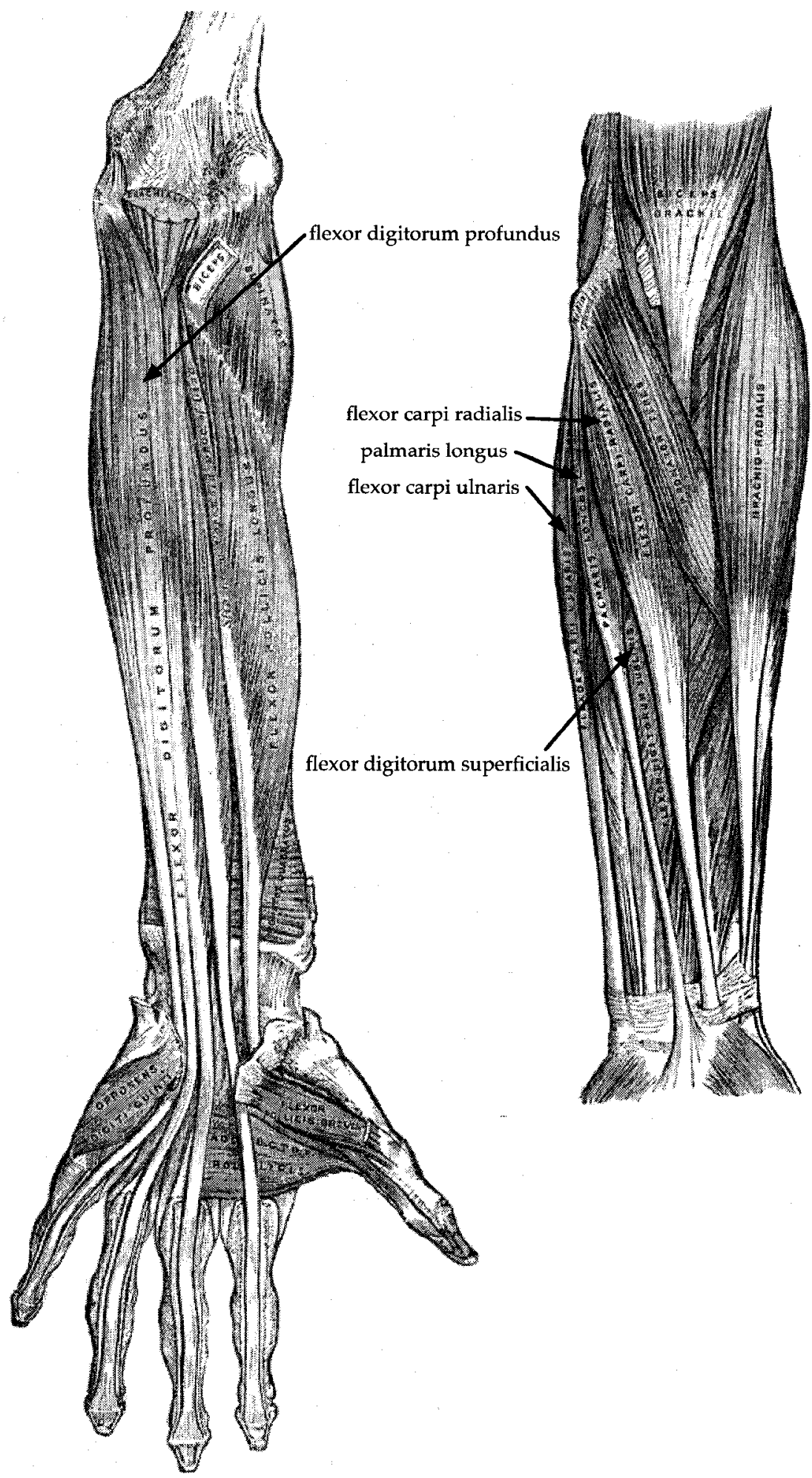

Figure 2.5: Muscles of the forearm, front view. Left image shows the deep muscles, right image shows the superficial muscles. [Figure adapted from Gray, 1918 [27]] 


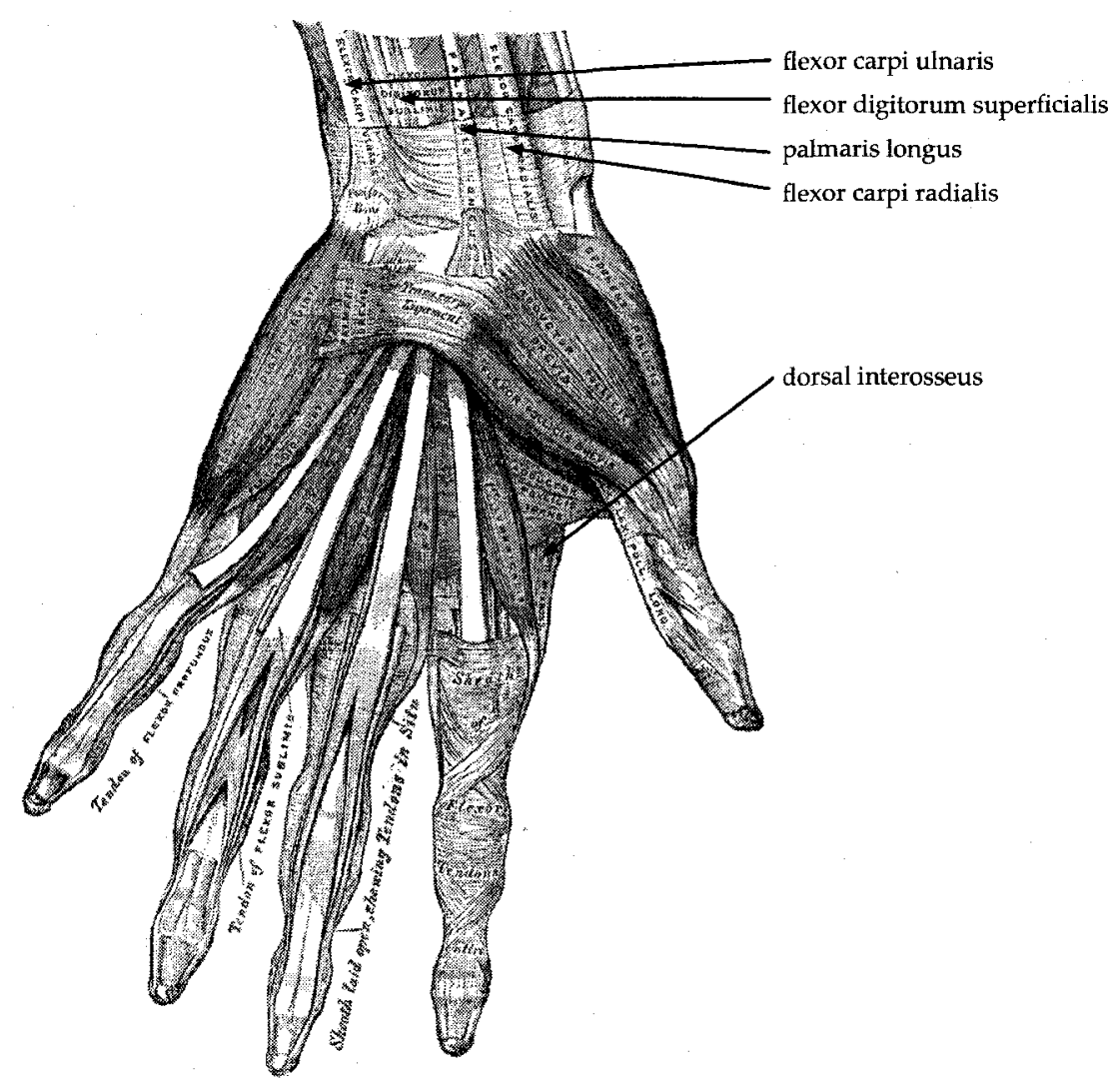

Figure 2.6: Muscles of the hand, posterior view. Tendons from forearm muscles also labelled. [Figure adapted from Gray, 1918 [27]] 
The sliding filament theory was developed separately but simultaneously by A.F. Huxley and H.E. Huxley in 1964 [28]. It states that the active shortening of the muscle results from the relative movement of the actin and myoisn filaments past one another. The force of contraction is developed by the movement of the myosin heads, also known as cross-bridges, which bind to the actin filaments and pull the myosin along. This pulling action has been described as similar to that of a man pulling a rope hand over hand or to a person climbing a ladder. The action of the cross-bridges is not synchronized, so while some bridges are connected and pulling others are reaching ahead for the next binding site. The pulling action of the myosin filament along the actin filament shortens the muscle.

A motor unit is the smallest part of a muscle that can contract independently. It consists of a single motor neuron and all the muscle fibres that it innervates - a neuron can innervate a single muscle fibre or multiple muscle fibres. The motor unit is responsible for the ability of the muscle to have varying states of activation; as more contractile effort is required more motor units are activated by their neurons, therefore the muscle as a whole has a higher level of contraction. Although the muscle fibre acts in an all-or-nothing fashion when contracting, the muscle as a whole has varying degrees of contraction.

The motor unit is activated by the neuron by which it is innervated, the electrical signal that is created initiates the contraction through the excitation-contraction coupling. This is a chemical process that requires a high concentration of calcium ions. As long as their concentration is high enough, the contraction is maintained because the calcium inhibits the troponin-tropomyosin system that causes relaxation in the muscle. The calcium ions are pumped away using the body's supply of ATP (adenosine triphosphate), the body's main source of energy. This causes the calcium to dissociate from the troponin, thus troponin's inhibitory effect on the contraction is restored. When the troponin bonds with the tropomyosin instead of the calcium, the actin filament slides back across the myosin filament, which lengthens the muscle in relaxation. 
When a muscle fibre is stimulated by an action potential from the motor neuron that innervates it, it produces a single twitch response; this is the smallest muscular response recordable. The twitch consists of: a latency period when the elastic components are stretched to remove slack; a contraction time, during which tension is built up from nothing to the peak tension; and a relaxation time, during which the tension drops from the peak tension back to zero. An action potential lasts only 1 to $2 \mathrm{~ms}$, but a twitch lasts about $150 \mathrm{~ms}$. It is possible to have a second action potential stimulate the muscle before the twitch response from the first action potential is complete. If the second action potential occurs after the latency period, it also induces a twitch response. The tension generated by the multiple twitches is the sum of the tension response from each individual twitch. This summation continues until the muscle reaches a maximal frequency of stimulation and the contraction reaches a maximal tension; this is called tetanic contraction (Figure 2.7).

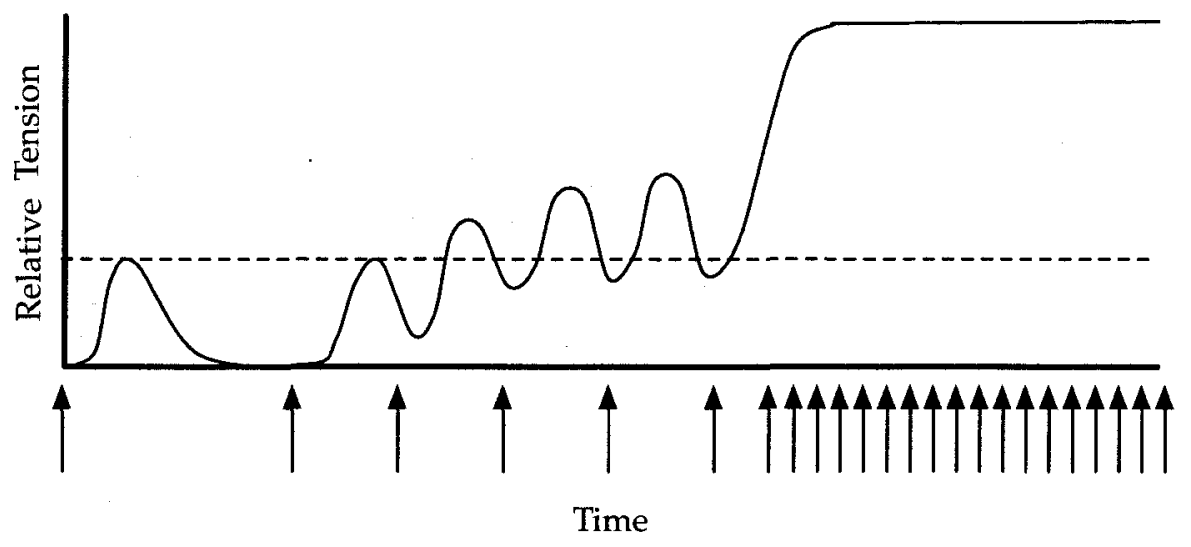

Figure 2.7: Muscles produce a twitch response to a stimulation (denoted by arrows). As the frequency of stimulation rises, the twitches overlap and their tensions are summed. This occurs until a maximal frequency when the muscle reaches its maximal contraction, known as tetanic contraction. [Figure adapted from Nordin and Frankel, 2001].

The ability of a muscle to contract is dependent on the availability of ATP - the energy source for contraction. At a high frequency of stimuli, the muscle cannot produce a sufficient amount of ATP to sustain contraction. At this time, the tension begins to decline as the inadequately-supplied sarcomeres are forced to stop contracting. Eventually all contraction 
ceases and the tension is reduced to nothing, as shown in Figure 2.8. If the frequency of the stimuli is low enough the contraction can be sustained for a long time; the critical frequency is dependent on the rate of production of ATP.

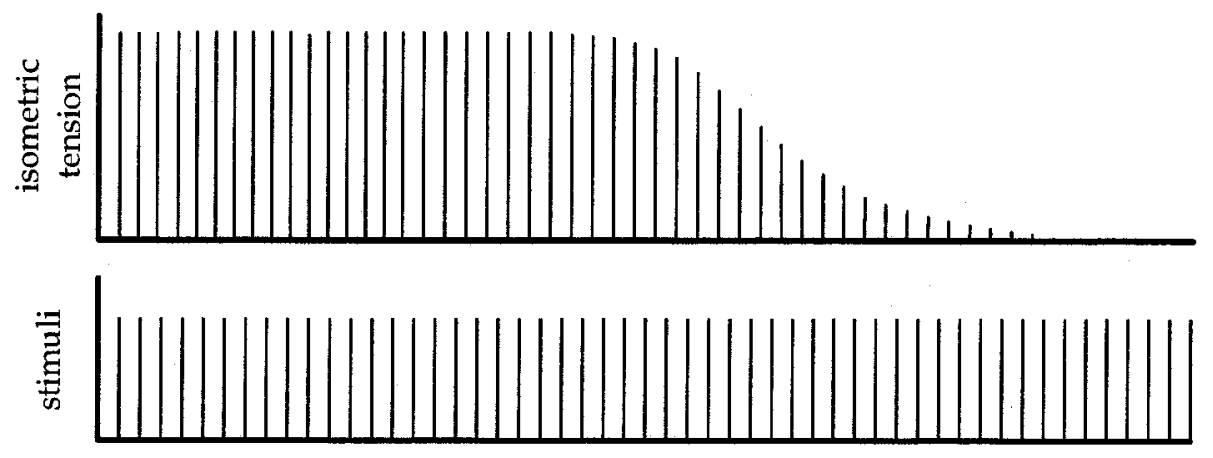

Figure 2.8: Fatigue in a muscle contracting isometrically. After prolonged stimulation the muscle is no longer able to produce enough ATP to sustain its contraction. The creation of tension is stopped when the muscle is no longer able to contract. [Figure adapted from Nordin and Frankel, 2001]

ATP is available from three mechanisms: creatinine phosphate, oxidative phosphorylation, and substrate phosphorylation. Creatinine phosphate is the fastest method of supplying ATP to the muscle. Enough ATP is stored in this form for about 100 twitches, which allows time for the phosphorylation to occur and generate a more sustainable supply. Oxidative phosphorylation is an efficient but slow means of ATP production, it is able to produce 38 ATP molecules per glucose molecule. Substrate phosphorylation, on the other hand, is inefficient but fast, producing only 1 ATP molecule per glucose molecule consumed. Oxidative phosphorylation is an aerobic process, meaning that it requires oxygen, which may be in short supply during heavy exercise, thus limiting its production capabilities. Substrate phosphorylation is an anaerobic process, meaning that it occurs in the absence of oxygen. However, it is unable to supply enough ATP to sustain a contraction on its own.

If the muscle is given a rest from contracting, the ATP supply increases and the muscle is able to undergo contraction again for a brief period before again submitting to fatigue. 
Muscles consist of either type I, type IIA or type IIB fibres which are specialized for either fast or slow muscle twitches. Slow-twitch muscle fibres (type I) are used for prolonged, lowintensity work because they are difficult to fatigue but produce little tension. Fast-twitch muscle fibres (type II) are divided into aerobic (type IIA) and anaerobic (type IIB). Type IIA fibres are used for relatively long periods because they are able to sustain lengthy contractions, however, they do fatigue eventually. Type IIB fibres lack the developed blood supply of the other two fibre types and so are referred to as white muscle. They are able to create a high level of tension, but only for short periods of time. 


\section{Chapter 3}

\section{Literature Review}

\subsection{Historical Piano Biomechanics}

The biomechanics of piano playing has been studied since the 1920s. In 1929, Ortmann published The Physiological Mechanics of Piano Technique [29] and in 1930 Bernstein and Popova published a Study of the Biodynamics of Piano Playing [30]. Both works were revolutionary because of their scientific methods and discussions.

Ortmann executed "an experimental study of the nature of muscular action as used in piano playing, and of the effects thereof upon the piano key and the piano tone". [29] For this he conducted an extensive series of experiments using considerable laboratory equipment including a cyclograph ${ }^{1}$, a roentgenogram ${ }^{2}$, electrical sensors mounted under piano keys and other devices he invented - such as a rotating drum that records the vertical movements of a player's finger. He measured range of motion of each upper extremity joint; explained physiological concepts, such as the role of muscles in performing more than one motion and that more than one muscle is involved in the motion of each joint; explained principles of mechanics, including forces and fulcrums; and analyzed various playing exercises, including scales, arpeggios and tremolo.

The result is a compilation of directives for piano playing based on mechanics and physiology. Some of his conclusions are: (1) that even the simplest movement involves multiple

\footnotetext{
${ }^{1}$ A method of motion capture whereby the positions of lightbulbs attached to a moving object are recorded on a stationary photosensitive film

${ }^{2} \mathrm{~A}$ photograph made using x-rays
} 
muscles that must be coordinated; (2) that equal and opposite movements do not require equal and opposite muscular movements since not all muscles have equal power and some actions must act against gravity while others act with it; (3) that muscles can be active even if no motion is visible because muscles can act antagonistically to each other to hold a joint in a fixed position; and (4) that each muscle has multiple functions, meaning that each plays a role in various movements not just one.

The following year, Bernstein and Popova set out to answer the question: "do changes in the tempo of a movement influence its construction and dynamics?". They employed kymocyclography ${ }^{3}$ to record performances of octave strikes at varying tempo and force of key strike (i.e. dynamic level). From this recording they calculated accelerations, joint reaction forces and muscle moments, which were analysed using free-body diagrams and knowledge of motor control. Although the sophistication of the equipment is limited by today's standards, the methods of analysis are still applicable.

Their results demonstrate that there was a change in movement caused by variations in tempo. At low speeds, the motor control scheme was described as "isolated impulses", and on the graph of muscle moments the chord playing showed up as bursts of activity with long periods of inactivity separating them. At medium speeds it acted as a "complex pendulum", and the muscle moment graph showed a double-peaked oscillations for the wrist and elbow. At high speeds the control scheme acted as a "simple pendulum" and the muscle moment curves for the elbow and wrist were single-peaked oscillations.

That is, at medium and low speeds there were two active effectors, that of the wrist and the elbow, while at high speeds only the elbow was active and the wrist became a passive element. The muscle moments for the shoulder were highly erratic, as if the motion of the elbow and wrist affected it. Another way of viewing this is that the movements appear to be segmented at low and medium speeds but appear continuous at high speeds, when the

\footnotetext{
${ }^{3}$ An improved method of cyclography whereby the positions are recorded on steadily-moving photosensitive film to allow the analysis of fine movements
} 
movements of the elbow seem to force those of the hand. Two major conclusions from this work are: (1) the wrist and elbow are a tightly coupled biomechanical linkage, while the shoulder maintains much more independence, and (2) motor control schemes vary depending on the tempo.

These early works were the basis of biomechanical research of piano playing for about 50 years until interest in the topic was renewed. The work of Ortmann, Bernstein and Popova is still relevant in modern discussions of piano biomechanics, however, some conclusions from their work are questioned because of assumptions that have been discarded with an improved understanding of physiology.

\subsection{Injury Analysis and Risk Factors}

Injuries occur in $30-60 \%$ of piano players at a professional level at some point in their career. A contributor to this high incidence rate is that piano teachers are not trained in biomechanics and physiology; faculty at leading conservatories generally focus on teaching repertoire and career management rather than technique and injury-avoidance [31]. Another level above this is the problem that there is no consensus on the cause, diagnosis and treatment for overuse injuries and, on top of this, many of the proposed causes await scientific validation [31].

The physical problems of musicians have been classified into five categories: overuse syndrome, tendonitis and tenosynovitis, nerve compression syndrome, thoracic outlet syndrome and focal dystonia [32]. The focus in this thesis is injuries due to overuse syndrome, also referred to as repetitive strain. The term "overuse" is debated among pedagogues, some argue that the injuries are due to a "misuse" of the instrument rather than "overuse" of their upper extremities [18].

The most prevalent sites of injury have been reported as the wrist [21] (36.6\% [20] and $49.1 \%[17])$ - especially the right wrist [33], the hand $[17,21]$, the spine $(56.5 \%[17])$, the forearm [21] and the upper extremity (31.7\% [17]). 
Causal information is often either anecdotal or experiential. The most commonly cited causes of injury are repertoire [34], practice habits [34-36] and technique [31,34,35]. Although they are the causes most commonly attributed to injury, it is not a unanimous accusation:

\begin{abstract}
"Physical injuries, unless they are caused by organic disease, are almost always due to persistent use of muscles after extreme fatigue has set in, or to inordinately high degrees of contraction. The notion that they are due to generally mistaken technical methods, as theorists are wont to declare, is not grounded in physiological fact. The kind of movement that a muscle produces has no effect on its well-being. The bad effects of bad technique are musical, not physiological." [37]
\end{abstract}

The demands of the repertoire have increased greatly as piano performers evolved from amateurs to professionals during the romantic period. The music became more technically demanding as careers were developed and more time was devoted to the study of the piano. Clara (Wieck) Schumann is an example of an early professional pianist [38], whose career was originally managed by her father and included performance, composition and teaching.

With respect to practice habits, no relationship has been found to correlate overpractice with injury [21], although increased practice time for one practice session [31] is reported to be a factor. Sudden changes in the intensity, type or duration of practice, such as after a vacation, are considered injury-inducing [35]; changes in type of work, hours and training are factors in creating inflammatory conditions in industrial workers and athletes [34]. Additionally, muscle-building exercises [36], playing through pain [35] and insufficient warm-up [35] are suggested as risk factors.

With respect to technique, a general rule is to maintain physiologic motion [33], that is, to remain within the normal ranges of motion for all joints. Based on force and moment measurements of the hand and wrist, An and Bejjani [39] found the ideal range of wrist 
motion to be: a total of 60 degrees of wrist extension, 54 degrees of flexion, 40 degrees of adduction, and 17 degrees of abduction. The normal functional range of the wrist is 40 degrees of extension, 40 degrees of flexion, 30 degrees of adduction and 10 degrees of abduction. [40]

"Extraneous and excessive" movements [31,34] introduce intrinsic strain that is reported to be a factor. EMG data shows that in females the intrinsic muscles of the hand are excessively loaded during piano playing [41]. Unnecessary muscular tension [31] is deemed inefficient and force-creating positions [42] cause undue strain, so both are considered causes of injury.

Specific hand, wrist and finger positions are also mentioned as either increasing or decreasing the risk of injury. Some movements that are considered risk factors are: exaggerated finger independence [34], a low wrist positions [34], the maintenance of a large hand span [43], and using a wide range of wrist motion [43], specifically ulnar deviation and wrist extension [44]. A curved finger position $[42,45,46]$, such as that shown in Figure 3.1, is thought to maintain a neutral wrist position thereby lowering joint and tendon forces and resulting in a lower incidence of injury. Joint and tendon forces have been shown to be dependent on the angular positions of the finger joints, the length of the finger segments and the keystrike force. [9]

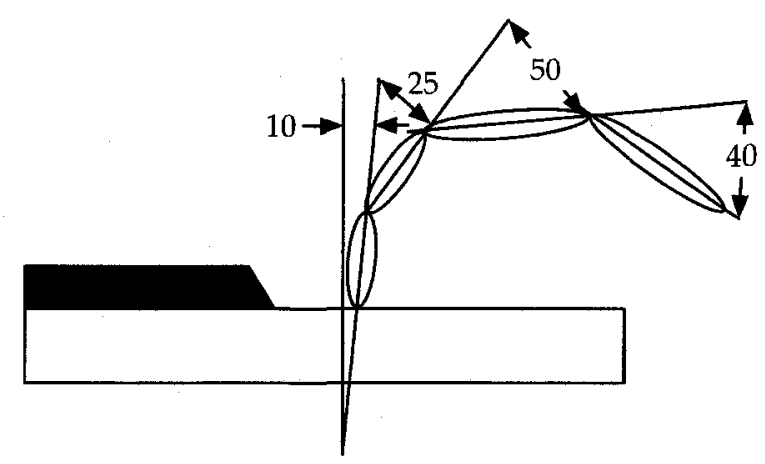

Figure 3.1: This curved finger position should reduce the risk of performance-related injury [42]. The numbers $(10,25,50,40)$ indicate the relative joint flexion angle for each joint (key contact angle, DIP, PIP, and MCP, respectively) [Figure adapted from Harding et al., 1993]. 
Other risk factors attributed to overuse injuries are:

- Anatomical limitations including repetitive tendon excursions across multiple joints [47], the interconnections of muscles and tendons, and the small joint contact areas in the fourth and fifth fingers which produce high stresses [9]; the anatomical limitations have been considered a factor since Schumann damaged his tendons trying to strengthen his fourth finger [48];

- Physical traits of the piano player [35] including hand size [18] and joint mobility [34] especially maximal wrist flexion, individuals were found to be more resistant to injury when they could perform maximal wrist extensions [19]; a positive correlation has related joint mobility and finger spans with the success of piano players, but hand size and shape were not related [49];

- Poor motor planning including muscular recruitment of small muscles as opposed to large muscles [44];

- The instrument [31] including the keyboard configuration and the weight required to play, for example Horowitz used a customized piano with lighter keys and was able to play without injury using an unconventional finger shape [42];

- Playing style including loud playing [45], forceful playing $[45,46]$ and playing with an unsustainably high energy expenditure [50];

- Virtuosity [31] including repertoire development, anxiety and stress; and

- "The process of music-making itself" [23]

\subsection{Piano Technique}

Piano techniques can vary significantly or can have only nuanced differences in their fundamental principles. Schools of pedagogy give a detailed discussion of technique, following the approaches of specific pedagogues, such as Suzuki, Alexander or Taubman. Suzuki has a significant impact on piano teaching, but provides methods of instruction for the young 
and not specific instruction of technique. Teachers who follow the Suzuki method present their own technique to the students. Alexander was in fact an actor who never played the piano, but developed a method of movement that has been adapted to many areas, including piano playing. Taubman has concentrated on the rehabilitation of injured pianists and has created a technique designed to accomplish this goal.

Due to a general lack of physiological knowledge in piano pedagogy, there is a reliance on metaphor and imagery to convey information between teacher and student. An example is shown in the following quote from Fink:

"Pronation and arm extension have a natural affinity, even though it is possible to do one without the other. Executed together, which in this exercise here feels like pressing and turning imaginary thumbtacks, clockwise with your left thumb, counterclockwise with your right, they produce an assertive, thrusting gesture. When boxers punch, their arms pronate ${ }^{4}$ as they extend, the pronation increasing the impact. Pronation increases the impact for pianists too." [5]

Another is this imagery from Schneiderman:

"The arm is buoyantly floating over the fingers, just as in walking." [51]

Generally, there are two methods used to teach movements. The first is to break a movement down into smaller motions and then unite them; Taubman [52] employs this technique when explaining double rotation. Although the desired action is one fluid movement, she introduces the concept as two motions: a preparatory swing phase and a "free drop" playing motion. The second method is to exaggerate a movement and then scale it back. For example, after a lengthy description of what he calls the "springing wrist", Bernstein states:

"In truth the springing wrist is never used for slow, detached playing; nor is it ever necessary to pull your hand back 5 or 6 inches from the keys. Yet in order

\footnotetext{
${ }^{4}$ It should be noted that boxers do not pronate during punching, but medially rotate from the shoulder. The difference is that boxers rotate the entire arm from the shoulder as opposed to pronation which is rotating the forearm from the elbow.
} 
to give you the sensation of a springing wrist, it was necessary to exaggerate this choreographic movement." [53]

A review of 18 pedagogical approaches, ten of which follow the Suzuki method, has revealed that there are both consistencies and inconsistencies in teachings - even within one pedagogical school or from the same pedagogue. Six examples of the inconsistencies between approaches are presented.

1. Forearm rotation: A pronation/supination movement of the forearm, from the elbow to the fingertips. It is a necessary component of three approaches [53-55] but is to be avoided in one approach [56].

2. Posture: The back is to be relaxed and straight, but two approaches $[2,5]$ recommend that is be vertical while three $[3,56,57]$ recommend that it have a slight incline towards the piano.

3. Hand alignment: Aligning the hand with the forearm $[54,58]$ allows for no abduction or adduction of the wrist, while aligning the MCP knuckle line with the fallboard [5] requires abduction and adduction of the wrist. A further inconsistency is found because each approach advocates the alignment of a different finger with the forearm: the second finger [59], the third finger [53] or the fourth and fifth fingers [60].

4. Firmness of arm: Many adjectives are used to describe the "firmness" of the arm, including firm [2,53], (not) tense [2], relaxed [2,3,57], (not) stiff [2], buoyant [51], floating [51] and flexible [53,61].

5. Wrist height: Two wrist heights are mentioned. One is level with the hand and forearm $[4,54,59,62]$, uniting the limb segments; and the other is "comfortably" low [53].

6. Playing style: Two contradictory playing styles are proposed: weighted playing [4, $55,59,60,63]$ and playing with independent, articulated fingers [51,53,61]. Both are 
endorsed by Gieseking, who first emphasizes a weighted playing with firm fingers that simply target the desired keys:

"Bend the arm, keeping the elbow, wrist and fingers in a fixed position but free from stiffness. The fingers must be firmly set in order to strike the desired keys.The arm should fall loosely from the shoulder joint; the fingers should perform the function of aiming at the respective keys without unnecessary maneuvers." [64]

and then later contradicts this technique when he advocates the independence of the playing fingers:

"In my opinion the development of fingers should be encouraged, since it is the point of departure in the attainment of a good keyboard technique. The student should be taught the fundamental principles of finer technique from the very beginning." [64]

There are also some consistencies that appear in this comparison of techniques. To be considered a consistency no approaches recommended a contradictory posture or method. This does not mean that it was recommended by every approach, some simply did not state a preference in their descriptions.

The four aspects of piano technique that were generally agreed upon are:

1. Hand position: Use a naturally curved hand position, which occurs when the hand and arm are hanging loosely at the side of the body. $[3,4,29,51,54,57,59,60,63]$

2. Flexible wrist: The wrist must be flexible to respond to hand and arm movements $[29,51,58,61,65,66]$, which may include rolling [2,60,63], light bending [3, 4, 63], undulations [53] or circles $[4,63]$.

3. Contact area: The fingers should play on the "fatty cushions" or "pads" rather than on the fingertip [2,3,53,63-65]. This increases cushioning effects and tactile feedback 
during playing.

4. Hand shape: The "bridge" should be the highest part of the hand's arch. $[2,53,54,59]$ The "bridge" refers to the arch of MCP joints.

\subsection{Evaluation of Piano Technique}

Piano technique has evolved based on current physiological knowledge and the constraints of the instrument; as suggested in Section 3.2, further changes to the instrument may be required to eliminate the injury-inducing aspects of piano playing. For example, in the 19th century, the modern piano gained more string tension, a heavier action and bigger sound, which led to the introduction of wrist motion and arm weight into the technique [49]. Musicians' manual skills have also become increasingly refined over the past three centuries. Whether this is due to longer practice sessions, specialization at a younger age or other factors, such as improvements in technique and teaching strategies, remains in question [67]. Each of these is potentially responsible for increasing manual dexterity, but each also has the potential to be the cause of performance-related injuries.

Until technique is ruled out as a contributor to injuries, its development should continue, and it seems that there is general agreement to this effect. The objective is therefore to determine which aspects of technique are improvements and which are regressions. Identification of these characteristics promotes technique development by supplying goals: minimize the degrading aspects and keep the healthy ones. Wristen has suggested the following goal:

"Develop a technique characterized by integrated, coordinated motions, so that forces are distributed throughout the anatomy instead of being localized." [43]

Some goals can be extracted from the review of risk factors of overuse injury. These include:

- minimize the joint and tendon forces;

- minimize the need for a large hand span, such as during arpeggios and trills; 
- minimize the action of finger independence;

- minimize muscular tension;

- minimize wrist motion, especially in ulnar deviation;

- minimize intrinsic strain; and

- keep the wrist level with the hand.

Current studies with the goal of evaluating piano technique are in general agreement for the goals of improving piano technique. Harding et al. $[9,42]$ used the goals of minimizing the joint reaction forces and the tendon and muscle tensions. Chung et al. [33] use the goal of minimizing wrist motion. Jabusch [68] looked at reducing tendon tension and the resultant forces in the finger joints. Wristen [35] suggests the main objective should be to minimize tension.

Other goals have been used in these studies as well. Hmelnitsky and Nettheim [69] used the goal of minimizing muscle tension, but only with respect to the forearm extensors, and An and Bejjani [39] minimized energy, force and jerk.

There appear to be two main goals of current studies: finding the optimal finger shape, and comparing weight playing to other playing styles. Bejjani et al. [70] and An and Bejjani [39] also included hand shape in their postural analysis.

In 1989, Bejjani et al. [70] compared the three hand positions shown in Figure 3.2: (1) a flat hand and extended fingers; (2) an arched hand with rounded fingers and a slightly flexed wrist and (3) a quasi-right angle flexion of the MCP joints and slight adduction of the wrist. The positions were compared based on surface electromyography ${ }^{5}$ (EMG) of eight muscle groups and three-dimensional spatial analysis. Most of the muscle groups showed no trends. The curved position required the most abduction-adduction in the MCP, while

\footnotetext{
${ }^{5}$ The monitoring and recording of the physiological signals in the muscles; gives a measure of muscle activation.
} 
the flat position required the least. Additionally, the arched position showed the greatest amount of abduction-adduction of the wrist.

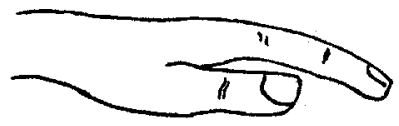

a

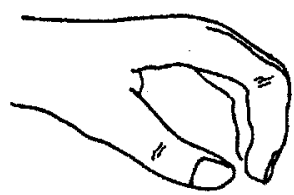

b

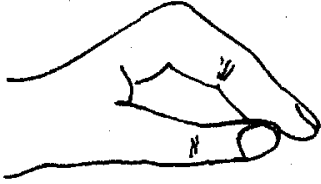

C

Figure 3.2: The three hand positions compared by Bejjani et al. (1989), (a) a flat hand with extended fingers, (b) a curved hand with curved fingers and (c) an arched hand with a high degree of flexion at the MCP joint.

In 1990, An and Bejjani [39] expanded on this work with the hypothesis that optimizations of energy of a movement, force of a movement and smoothness of a movement would be obtained by minimizing velocity, acceleration and jerk $^{6}$, respectively. The energy, force and motion costs were calculated for each of the three playing postures at each measured joint: shoulder, elbow, wrist, MCP of the third and fifth fingers, PIP and DIP of the third finger. The conclusions were that the first two playing postures were generally superior at minimizing the energy and force costs and that therefore there may be a greater risk of injury with the third posture.

In 1989 Harding et al. [9] created a finger model to optimze joint and tendon forces during piano playing. The mathematical model consisted of a free-body diagram of a static index finger with four variable angles: key contact angle, DIP flexion, PIP flexion and MCP flexion. The goal was to find the finger shapes that minimized joint forces at the MCP and DIP joints and tendon tension for the FDP (flexor digitorum profundus, the muscle that flexes the index finger).

The finger positions for minimizing the DIP joint were selected arbitrarily, and it was found that higher DIP and PIP flexion angles and a lower key contact angle decreased the

\footnotetext{
${ }^{6}$ Jerk is defined as the third derivative of displacement
} 
DIP joint force by over $50 \%$. For the MCP joint force, the contact angle and DIP forces were held constant while MCP and PIP flexion angles were varied. The minimum force was found using high MCP and low PIP flexion angles, resulting in a finger that is almost perpendicular to the key. The FDP tendon force was calculated as a function of DIP flexion angle and the key contact angle. The minimum FDP tendon force was found with a low key contact angle and a high DIP angle. This results in a finger that is almost perpendicular to the key and is not useful for piano playing. An important finding was that when the joint and tendon forces were decreased, there was no corresponding increase in forces elsewhere in the finger.

Additionally, the forces during keystrike were derived from MIDI velocity measurements at different dynamic levels and with both legato and staccato touches. It was found that there was a peak force that appeared after the key had been fully depressed and that the elimination of this excess force application may reduce forces in the hand, thus reducing the risk of overuse injuries.

In 1993, Harding et al. [42] expanded on the mathematical finger optimization. The model was still restricted to sagittal plane motion, but now included ten tendon tensions instead of one and three joint reaction forces instead of two. The motion of the finger was measured as well, using a spatial motion analysis system that detected the position of five light-emitting diodes (LEDs) mounted on the MCP, PIP and DIP joints as well as near the fingertip and on the key. The force measurement was based on MIDI data measured by the instrument; the instrument was calibrated using a force transducer over a range of key velocities.

The numerical optimizations performed were to minimize (1) each joint reaction force, (2) three of the tendon and muscle tensions (INT, FDP and FDS), (3) the sum of the joint reaction forces and (4) the sum of the tendon tensions. Two finger positions, a best and worst case, were found for each optimization. A general finding from these results is that minimizing the joint reaction force at the MCP and maximizing flexor tendon moments 
about the joints will minimize all finger forces. That is, use a curved finger with a large MCP flexion angle, as shown in Figure 3.1.

Wolf et al. [45] found a similar result in 1993. In this study, the finger positions analysed were based on video-captured images of the finger depressing a key while subjects played a selection from Mendelssohn's Songs Without Words. Ten notes that represented the full dynamic (loudness) range were selected for analysis. Finger segment lengths, keystrike force and finger flexion angles were input into Harding's model based on the experimental results, and joint reaction forces and tendon forces were calculated. Each piano player used a variety of finger shapes while playing. Visual inspection of the results shows that the curved finger with an almost perpedicular contact angle minimized DIP joint reaction forces and FDP tendon forces.

In 1987, Hmelnitsky and Nettheim [69] attempt to compare the weight-based and traditional playing based solely on observation and descriptions of which muscles are used. The authors propose that in traditional playing the wrist extensors and elbow flexors are used excessively to withdraw the hand from the keyboard, but that in weight-based playing, gravity is used to extend the wrist and finger flexors are used to depress the keys. The minimal effort by the wrist extensors in weight-playing is viewed as beneficial since pain often occurs at the wrist. Additionally, it is suggested that the finger is extended with the aid of the interosseus hood, a conglomeration of interosseus muscles. The conclusion is that since the fingers need to be lifted only a small distance to withdraw from the key, the large movements in traditional playing are unnecessary and are the cause of overuse syndrome.

Chung et al. [33] compared weight playing with traditional playing in 1992 by measuring the average range of motions of the wrist using electrogoniometers. Traditional players were found to use a greater range of motion in both flexion-extension and abduction-adduction. The frequency domain information was converted into a measure of energy using Parseval's theorem. Traditional players were found to have greater energy expenditure in abductionadduction and weight-playing pianists had greater energy expenditure in flexion-extension. 
In comparison with activities of daily living, either method of piano playing was found to have an increased range of motion in both flexion-extension and abduction-adduction.

It is interesting to note that in 1930, Bernstein and Popova [30] discredited weighted playing because the ideal weighted motion - to depress the keys using passive motion of the arm - did not occur during playing. Even among players using the weight technique, active motion was always present.

Others have been contributing to the analysis of piano technique and injury-prevention by creating guidelines to help integrate the diverse fields involved in this interdisciplinary work. To bridge the gap between piano pedagogy and the medical sciences, Wristen [43] created checklists for use during visual observation of a player's technique. Additionally, Meinke and Lagendor [34] proposed that the medical examination of injured pianists include visual observation of the player at the piano and the medical history should include practice and performance histories.

\subsection{Stiffness Determination}

The following is a discussion of the methodology for stiffness determination. A more extensive discussion of stiffness is given in Chapter 4 .

Determination of wrist stiffness, a joint stiffness, is generally based on measurements using an endpoint approach. This is partially due to the fact that many limb functions are endpoint-dependent [71], such as placing the hand on a target. It is also due to the ease of applying a force to the endpoint. For endpoint measurements a force is applied to the endpoint and position measurements are taken at the endpoint and at each of the joints.

Stiffness depends on the magnitude and direction of the applied force as well as the posture of the joint [72]. Gomi and Kawato [8] used an algorithm that calculated the stiffnesses independent of position. For joints in the upper extremity, a manipulandum (Figure 3.3) is used to apply forces at the endpoint. A manipulandum is a two-link robot with a joystick 
handle which can be used to determine stiffnesses for the wrist, elbow and shoulder. Since the hand grips the manipulandum it does not generate joint stiffnesses for the knuckles.
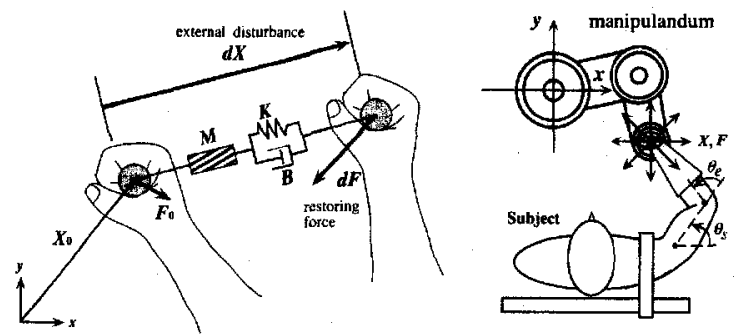

Figure 3.3: The manipulandum set-up used by Tsuji et al. (1995). The subject's wrist and hand were constrained to restrict joint movement and eliminate the effects of gripping the manipulandum, respectively.

Gomi and Kawato state five constraints for a manipulandum design:

"The manipulandum needs to be (1) fast and light enough to minimize movement interference, while also being (2) strong enough to transmit large forces, and (3) rigid enough to be controlled at high frequencies. It is also necessary to (4) support the human arm on a horizontal plane to be free from the force of gravity and to reduce fatigue. Additionally, (5) nonlinear forces due to manipulandum dynamics should be reduced so as not to disturb the arm movements." [8]

The hand can also be attached to the manipulandum using external bracing to eliminate any effects from grasping [7]. The manipulandum-hand construct can also be covered to eliminate any effect of visual feedback [7]. The manipulandum applies a force field, by a mechanical means such as motors [6]. The force field may be constant or variable, often including the application of disturbances. Any disturbances should be applied randomly to avoid prediction.

While the force field is applied, the subject performs a task. Two common tasks are: to move between two targets [8], for the determination of stiffness during a movement; or to keep the manipulandum steady within a biased force field $[6,7]$, for the determination of 
postural stiffness. The arm movements are constrained to the horizontal plane of motion using braces, platforms and the manipulandum itself.

The voluntary muscular response is known to take about $400 \mathrm{~ms}$ to become active. Therefore a measure can be taken of the reflex actions if the disturbance lasts less than $400 \mathrm{~ms}$ [7]. Additionally, the external force applied must be an appropriate magnitude. If it is too large, the stiffness will increase to prevent failure of the task and if it is too small there will be no reaction to measure [8].

For stiffness, step-like disturbances can be applied when measurements are taken only after the transient dynamic response. The impedance measurement includes the dynamic response assumed to include inertial and viscosity effects [7]. During a short disturbance that only includes reflex actions the inertia, viscosity and stiffness can be assumed to be constant.

Perreault et al. [71] developed a multiple-input, multiple output routine that implements stochastic perturbations instead of the step or ramp perturbations normally applied. The stochastic perturbations are random by nature and so reduce the effect of voluntary reactions. Additionally, they are able to test the entire stiffness field during one trial instead of requiring separate trials for each direction of interest.

The manipulandum records position data using potentiometers [6] or optics [7]. The joint angles of the arm are measured using a three-dimensional camera system [7]. The measurements of the applied force field and the position of the manipulandum can then be extended to calculate joint stiffnesses at the wrist, elbow and shoulder using a mathematical model of the limb.

The formula for stiffness can be condensed into matrix form as:

$$
\vec{F}=\mathbf{K} d \vec{x}
$$

where: 
$\vec{F}$ is the force vector;

$\mathbf{K}$ is the stiffness matrix; and

$d \vec{x}$ is the vector of changes in displacement

If no joint is constrained, the arm is represented as a three-link model: upper arm, forearm and hand segments with the joints at the shoulder, elbow and wrist. In some cases a joint can be braced to reduce the number of compliancies, thus simplifying the problem. In this case if the elbow was braced, the system would be represented as a two-link model: arm and hand segments with joints at the shoulder and wrist.

In the problem of taking measurements while playing the piano, the endpoint is occupied by the act of depressing the key. Jindrich et al. [72] encountered a similar problem while measuring stiffness in the finger during the task of tapping on a keyswitch. The force and displacement used for the stiffness calculations in this case were those applied by the finger on the keyswitch. The force was measured using a two-axis strain gauge force transducer and the displacement was the vertical displacement of the keyswitch, measured by an optical tracking system. The position of the finger was measured using miniature goniometers mounted on each finger joint. 


\section{Chapter 4}

\section{Clarification of Existing Terminology}

The pedagogical world and biomedical world have started working together to create a healthier environment for artists. However, expertise and language in the two fields are considerably different and thus the two fields are divided by a great expanse, which results in an imprecise use of terminology. The use of biomechanical terminology in the descriptions of piano technique is well-intended, but the meaning of specific terms, such as relaxation, becomes vague and varied. Four of the primary areas of confusion are in defining and fully understanding the terms: stiffness, relaxation, co-contraction and multi-joint issues.

\subsection{Stiffness}

The term stiffness is used in many disciplines with many different meanings. In the discussion of piano biomechanics the distinction between various uses is important. The concepts of muscle stiffness, joint stiffness and impedance are examined and differentiated.

\subsubsection{As a Measure of Modulus in Biomechanical Engineering}

The definition of stiffness varies with its application and field of study. Mechanical stiffness refers to a relationship between force and deformation of a solid material. A familiar application of the term stiffness is in the mechanical engineering use as a descriptor for a mechanical spring. In this sense it is a proportionality parameter, i.e. a constant, that relates the force and displacement of linear springs. This relationship is represented by the equation:

$$
k=F / x
$$


where:

$k$ is the stiffness;

$F$ is the force; and

$x$ is the displacement.

Not all springs are linear, however, and stiffness can also be used as a descriptor for non-linear springs. Here, it is a function of spring stretch and refers to the derivative of the force function. This relationship is represented by the equation:

$$
k(x)=d F / d x
$$

In biomechanics, muscles are often modelled as springs. The passive properties can be modelled using a spring that is constrained at one end; this is known as the variable stiffness spring model. Passive properties are the properties of the muscle at rest; these are the elastic properties of the connective tissues (Figure 4.1). The passive elastic properties vary based on the amount and geometry of connective tissues in the muscle.

Other more complex models of muscles exist that incorporate the active properties of muscle-tendon unit. These models, such as Hill's muscle models (Figure 4.2), include other mechanical elements such as dampers and force generators. The series elastic element represents the contribution of the tendons, the parallel elastic element represents the connective tissue contributions and the behaviour of the contractile element is modelled by the damper and/or force generator.

The total muscle stiffness is dependent on both the active and passive properties, as shown in Figure 4.3. The passive properties generate stiffness when a muscle is stretched beyond its resting length, it is the resistance of the connective tissues to being stretched. The active properties generate stiffness at lengths where cross-bridges can form, generally $60-180 \%$ of the resting length. Below $60 \%$ of the resting length there is too much overlap of 


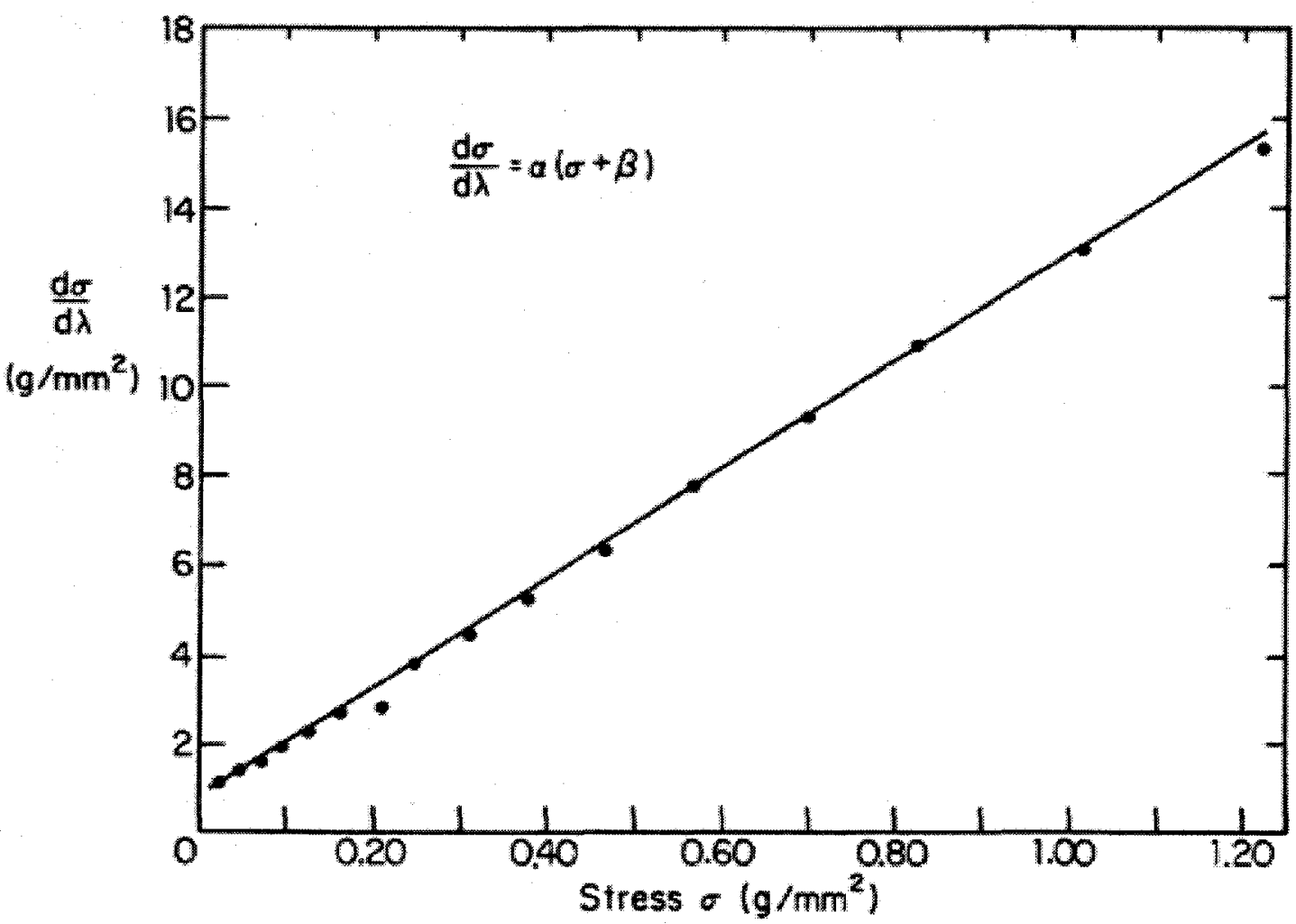

Figure 4.1: The passive stiffness of a muscle is based on the elastic properties of the connective tissues in the muscle-tendon unit. The passive stretch of a papillary muscle of a rabbit heart is shown here; the slope of the graph changes with muscle geometry and composition. [28] 


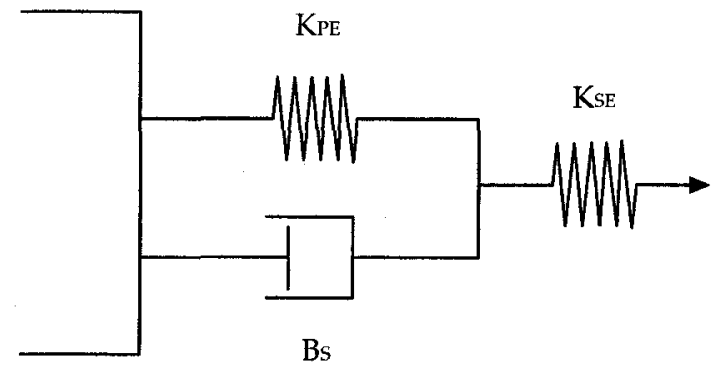

(a)

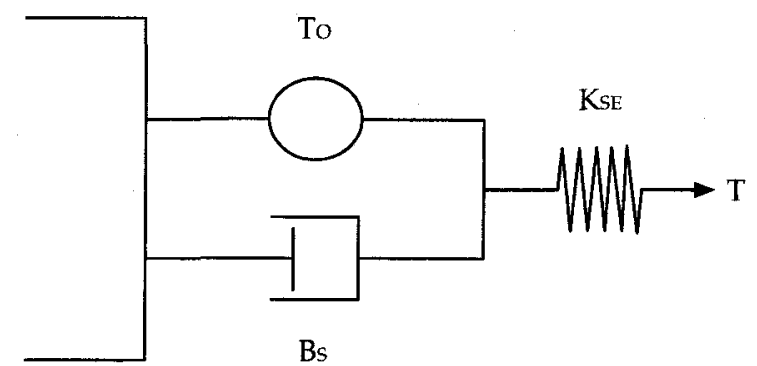

(b)

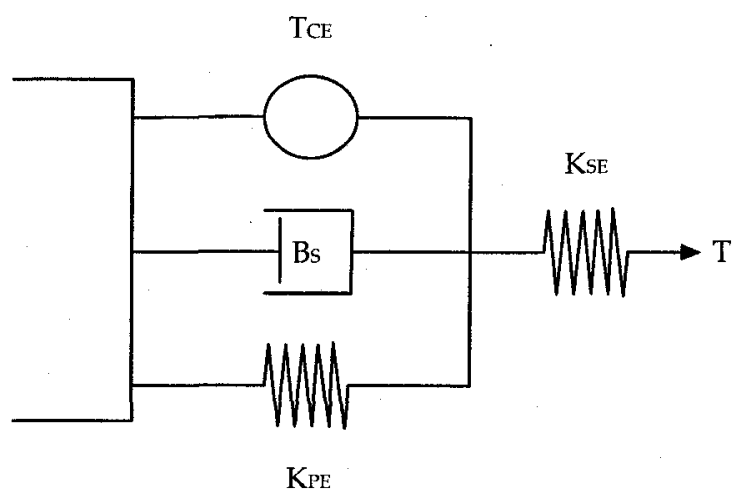

(c)

Figure 4.2: Hill's muscle models model the entire muscle and tendon unit using mechanical elements. (a) is the three-element model, consisting of series and parallel elastic elements $\left(K_{S E}, K_{P E}\right)$ and a damper $\left(B_{S}\right)$. (b) is the Simplified three-element model, consisting of a force generator $\left(T_{C E}\right)$, an elastic element $\left(K_{S E}\right)$ and a damper $\left(B_{S}\right)$. (c) is the fourelement model consisting of a force generator $\left(T_{C E}\right)$, series and parallel elastic components $\left(K_{S E}, K_{P E}\right)$ and a damper $\left(B_{S}\right)$. 
the contractile elements (sarcomeres) for cross-bridges to form and above $180 \%$ there is no overlap between contractile elements (sarcomeres) on which the cross-bridges could form. The shape of the active component is generally constant for all muscles.

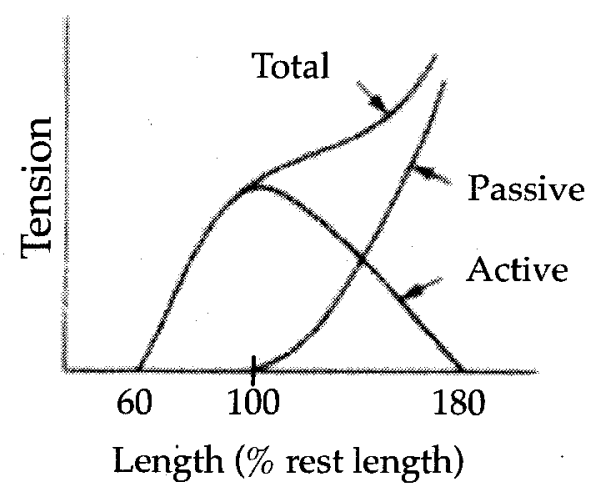

Figure 4.3: The total stiffness of a muscle is dependent on both active and passive components. The active component is from the contractile properties of the muscle and has a constant shape for all muscles. The passive component is from the elastic properties of the connective tissue and varies with the composition of the muscle. [Figure adapted from Nordin and Frankel, 2001 and Freivalds, 2004].

The active stiffness component includes contraction from both reflexes and voluntary control. The stiffness indicator generally used for active stiffness is EMG, a measure of the electrical activity of the muscles.

In most perturbation stiffness experiments, the reaction to perturbation includes the passive elastic component and active reflex responses but not voluntary muscular contractions. The uncontrolled response time is limited to $400 \mathrm{~ms}$, and so measurable displacements that occur in response to externally applied perturbations have a limited duration of $400 \mathrm{~ms}$, or subjects are told to "not intervene".

It is evident from Figures 4.1 and 4.3 that the length of the muscle is a factor in both the active and passive muscle stiffness, and therefore also in the total stiffness. The active stiffness of the muscle is dependent on the ability to create a contraction. 
Adaptation as a result of learning has been found to inversely correlate with muscle stiffness [73,74]; lower stiffness was measured during trained movements and higher EMG was recorded while learning new movements. The effects of learning are based on the adaptation of muscle cells to repeated movements. The adaptation consists of increasing the cross-sectional area of the muscle fibres, and some suggest that it is also due to a change in the fibre composition of the muscle [75].

The above discussion deals only with muscular stiffness, but there are various types of stiffness in biomechanics. Joint stiffness is the other type of stiffness that is important to this discussion.

Joint stiffness is the rate of change of the joint moment with respect to joint displacement [76]:

$$
\Psi=d M / d \theta
$$

where:

$\Psi$ is the joint stiffness;

$M$ is the joint moment; and

$\theta$ is the joint displacement.

It is possible, and common, to have multiple moments and displacements at one joint, in which case the joint stiffness becomes a matrix. It is a measure of resistance to joint displacement.

Joint stiffness is important for stabilizing a joint. Joints generally return to be in their relaxed, neutral positions but other positions are necessary for accomplishing tasks.

Joint stiffness and muscle stiffness are both internal stiffness. External stiffness exists as well. An example of external stiffness is impedance. Impedance is a measure of resistance 
to an applied force, but it is not specific to a muscle or a joint. For example, a driving point impedance measures the impedance at the point of an applied disturbance, which is called the driving point. If the driving point is at the wrist, the reaction to the driving point impedance measures the response from the wrist, but the measurement also includes responses from neighbouring joints and their muscles. Impedance is assumed to include dynamic responses due to inertia and viscosity as well as that of stiffness [7].

In biomechanical engineering, stiffness is a quantifiable property that has a range of possible values and does not fit into the on/off states attributed to it by piano pedagogy.

\subsubsection{Assessment of Several Uses in Pedagogical Literature}

In piano pedagogy, stiffness is generally viewed as negative, something that should be avoided, and the cause of injury (e.g. $[4,61,64]$ ). However, it is not possible to avoid stiffness in all muscles about a joint concurrently. The reasons for this are discussed in Section 4.2.2. Due to biomechanical principles, stiffness must be present in at least one muscle about a joint and in the maintenance of postures, stiffness must also be present in the joint itself. An interesting, and wrong, implementation of the term 'stiff' is the following:

"If one part of the body becomes fixed or stiff and ceases to contribute its share of the movement, we will probably still be able to play the piano." [77]

The major oversight is that parts of the body must become stiff so that they can contribute to movement. Movement is created at joints, which are rotated using muscles. Muscle contractions, which always produce stiffness, are required for the muscle to exert moments or forces about a joint and produce the desired motion.

Several terms are used interchangeably to describe stiffness in pedagogical literature; these include stiffness, tension and effort. Some examples of the use of these terms are included to give an indication of their use:

"Avoid rigidity, to maintain a flexible state at all times, to breathe and move 
physically with the music, and to use weight instead of pressure on the instrument so that the joints, muscles, and tendons are not over-used or stiff [will contribute to injury-avoidance]." [78]

"Bend the arm, keeping the elbow, wrist and fingers in a fixed position but free from stiffness. The fingers must be firmly set in order to strike the desired keys." [64]

"The range of effort in the elbow is about five times bigger than that in the wrist, while the range of effort in the shoulder is eight times bigger than in the wrist." [30]

In general, there are three types of reference to stiffness: those that are biomechanically correct; those that are ambiguous or biomechanically wrong; and those that are using stiffness in a purely conceptual way without reference to any anatomical structure.

The biomechanically correct stiffness references clearly refer to stiffness of either joints or muscles, which are appropriate uses of the term in a biomechanical discussion. It should be noted that because the pedagogue uses the term stiffness in a manner that agrees with biomechanical usage does not mean that their statements fully follow biomechanical principles. Some examples of usage from this group are given below.

"Claw Position is derived from the extended position by flexing the nail and midjoints inward (under) while the knuckle joints remain still. Be gentle, tightening your fingers no more than necessary to form the hands in this position. The long finger flexors, located under the forearms, control these actions by pulling through and tensing the wrists. Note that the thumbs also curve with their palm bones back, a position called claw thumbs." [5]

"Fingers curved tightly at the nail joint move the long flexors which connect into the arm and are a leading cause of tension and hurting in the lower arm. In my studio we often wave our fingers and talk of loose fingers." [63] 
"One reason this [forearm rotation] works so well is because when you are doing "A", you can't be doing "B," that is, if your forearm is rotating it can't be clenching [stiff]." [79]

The ambiguous stiffness references refer to stiffness of an entire limb. This is ambiguous because there are many muscles and joints in a limb and it is unlikely that the pedagogue would expect any piano player to stiffen every joint and muscle of the arm, for example. The pedagogue either is a dilettante in biomechanics or is simply not specific enough in their description. Some examples are included below.

"The arm is firm, but not tense or stiff." [2]

"The shoulders, head and back must be free of tension with the energy supporting the body ultimately over time coming from the feet." [59]

"Je parlerai maintenant d'une constante physique que nous ne devons jamais perdre de vue. Il s'agit d'avoir la paume de la main toujours vide, sans aucune tension interne même si les doigts ont de grandes distances à franchir." [61]

This last quote also is biomechanically wrong because to completely lack internal stiffness in the palm of the hand, no muscles could have any stiffness, either passive or active.

The conceptual stiffness references refer to stiffness without respect to any part of the body. This group is using stiffness in a purely conceptual way. A complete lack of stiffness in the body is impossible to achieve because there is redundancy at every joint - many muscles control the function of one joint and at least one muscle must have some build-up of stiffness, whether it is passive or active. Some examples follow.

"The body never becomes stiff when producing a musical sound." [80]

"At any level, the student should be relaxed and tension-free." [4] 
"The body already knows what to do; we need only permit the motion without tension, tightness or forcing (let it happen, not make it happen ). We learn to know the difference between intensity and tension - to express the former without the latter." [51]

"Piano technique had moved from stiff playing positions where water wouldn't spill from a glass placed upon a wrist, to technical approaches where freedom of movement or flexibility, relaxation and weight-playing became the norm." [81]

\subsection{Relaxation}

In describing piano technique, much emphasis is placed on the importance of relaxation during playing; Mark McCray even went as far as to say that relaxation is the key to preventing injury [78]. Monique Deschaussées emphasizes the importance of a relaxed state:

"Vivre dans cet état [un état de détente, de relaxation] est la préparation la plus indispensable pour jouer du piano plus tard sans effort." [61]

There are endless accounts of the term relaxation in piano literature, a few are included here to give an indication of their use :

"Those who relax and play with good hand position are less likely to develop physical injuries than those who play with tension." [78]

"Injury results from too much relaxation, too much tension." [78]

"Move in a way that there is nothing to relax from." [54]

"Each finger must be supported by a relaxed wrist and arm." [2]

The term relaxation is presented in both pedagogical literature and biomechanical engineering and the meanings of these uses will be discussed in the following sections. 


\subsubsection{As an Approximation for the Non-equivalent Terms of 'Stiffness' and 'Force' in Pedagogical Literature}

The bulk of pedagogical literature defines relaxation in one of two ways; as an antonym for either stiffness or force. In terms of stiffness, it is viewed as a muscle having a lack of stiffness or tension - this corresponds well with usage in mechanical engineering in which relaxation is described as the release of tension. In terms of force, it is viewed as lacking the ability to produce force.

For example, Doris Koppelman states:

"I prefer to think of letting go of extra effort and being ready to do anything easily than to think of relaxation." [82]

Koppelman relates relaxation to effort, or force production.

Conversely, E.L. Lancaster [78] and Robert Shannon [78] state that "too much relaxation or too much tension" can lead to injury in later years. Here Lancaster and Shannon associate relaxation to tension or stiffness of the muscle. Similarly, C. Liccardo [82] connects relaxation to tension in the statement: "At any level the student should be relaxed and tension-free".

These two meanings of relaxation are not equivalent; it is possible for a muscle to be stiff without producing an external force. In 1929, Otto Ortmann presented this to the pedagogical world:

"Absence of motion does not necessarily mean absence of muscular activity...antagonistic muscle groups can act equally upon a joint, setting the joint in a fixed position but not producing motion at the joint." [29]

In other cases the precise meaning of the term is unclear. For example pedagogues have stated "I emphasize a natural and relaxed body position" [82], and "The natural position of the hand with relaxed muscles (as when we walk)" [57]. 
Although it is the body of work as a whole that generally confounds the meaning of the terminology, some pedagogues have made the effort to clearly define their meaning,

"Relaxation should be treated as the comfort felt for coordinate movement, not a complete relaxation of all the muscles in the playing apparatus." [83]

and

"Students who are relaxed will play with lips slightly apart." [78]

To form a cohesive body of knowledge, it is essential to clarify and distinguish between the two most common meanings of relaxation in the pedagogical world; one that states that relaxation is the state of creating no external force and the other that states that relaxation is the state lacking in muscular stiffness. Since a clear and well-defined meaning of relaxation exists in biomechanical engineering, this is the logical choice for the pedagogical community to employ.

\subsubsection{As a State of Activation in Biomechanical Engineering}

In the realm of biomechanical engineering, relaxation is described as a state of (in)activation of the muscle [84]. That is, a state lacking neural input or activation. Neural excitation to the muscle causes a contraction to occur, so a lack of neural excitation is the state where the contraction is not occuring. Therefore relaxation can be approximated as the lengthening of inactive muscle or individual muscle fibres to their rest length and maintaining the inactive muscle fibres at rest length. Rest length is the length at which there is a maximum overlap of the thin filaments and the useful cross-bridge-forming portion of the thick filament to allow maximal cross-bridge formation [85]. Rest length typically yields the maximum tension when contracted isometrically. At shorter lengths, there can be interference from crowded, neighbouring cross-bridges and at longer lengths, there can be a decrease in the overlap between the thin filaments and the cross-bridge-forming portion of the thick filaments. 
A contraction is the ability of a muscle to create tension and occurs due to the presence of a stimulus that activates the muscle. Any contraction produces tension. There are various classifications of contraction, two of which are isotonic or isometric.

In an isotonic contraction, the muscle shortens under a constant external load, thereby exerting an external force on the load. In the case of flexion at the elbow, an isotonic contraction of the biceps muscle pulls against the weight of the forearm, which is the load. The muscle theoretically shortens at a rate that keeps the force constant, however since the moment arm of the muscle changes as the muscle shortens, the muscular tension must change as well. A true isotonic contraction does not exist in joint motion, but the idea is maintained that it is a contraction with a change of length while simultaneously exerting an external force.

There are two types of isotonic contractions that a muscle can undergo: concentric and eccentric. When the tension in the muscle has increased enough to overcome the resistance against the limb, the muscle can shorten thereby moving the body part and undergoing a concentric contraction, as shown in Figure 4.4. Conversely when the resistance against the limb is greater than the tension in the muscle, the muscle lengthens, undergoing an eccentric contraction, as shown in Figure 4.4. In an eccentric contraction the joint motion and the contraction occur in opposing directions.

In an isometric contraction, the length of the muscle remains constant throughout the contraction. In this case tension is still produced but no external mechanical work is performed. Instead physiological, or muscle, work is performed and energy is expended to perform this work; the energy is mostly dissipated as heat. During an isometric contraction, the muscle still acts against an external load and tries to shorten, but it does not overcome the load and create joint motion. Instead it produces a moment that supports the load. An example of an isometric contraction occurs whenever the body maintains a posture against gravity. Specifically, when sitting the back muscles contract isometrically to hold the back upright against the weight of the body, which is produced by the force of 


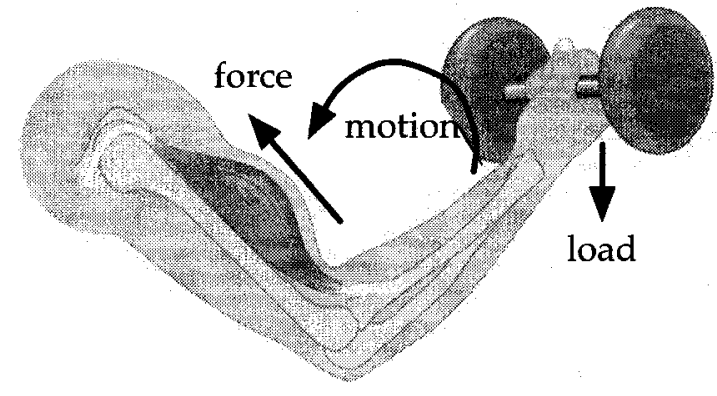

a

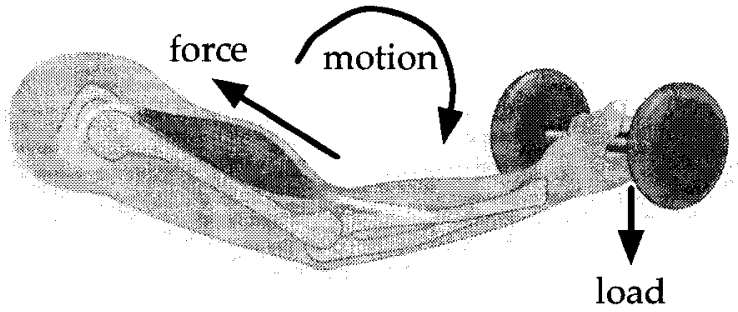

b

Figure 4.4: There are two kinds of isotonic contractions: (a) concentric contractions where the muscular force and the joint motion are in the same direction, the force overcomes the external load, and (b) eccentric contractions where the muscular force and joint motion are in opposite directions, the external load is too great for the force.

gravity acting on the body's mass.

The degree of tension produced varies with the type of contraction, as well as other factors, including the length at which it is held and the duration of the contraction.

"Muscle relaxation with movement [isotonic relaxation] may be achieved mainly by the excitation of descending corticospinal projections to produce active inhibition, whereas isometric muscle relaxation may be achieved mainly by the withdrawal of ongoing input to pyramidal neurons, mediated by the intracortical inhibitory neurons, which requires little preparation in motor cortex." [86]

The two disciplines agree on the definition where relaxation corresponds to stiffness. In this case, a relaxed state is one in which there is no contraction occurring. Every contraction creates a change in muscular stiffness, however not all contractions will produce a muscular force that will perform external mechanical work. A relaxed state can be assumed to have negligible muscle forces [87], although the logic cannot be reversed; a state lacking muscle forces is not always a relaxed state. 


\section{Joint Relaxation}

The above definitions only apply to muscular relaxation. Relaxation can also be viewed from the standpoint of a joint with multiple muscles acting on it. A relaxed position of the joint is the neutral position. This is the position at which the joint is 'loosest' or has the most give or play in it and can be approximated by the mid-range of the joint [88]. For example, if a joint has a 180-degree range of motion (ROM), the neutral or relaxed position will be at approximately 90 degrees. When injured, a joint will naturally seek this position because it allows the most room for swelling of the soft tissues [88].

From the standpoint of the multiple muscles acting on a joint, each muscle cannot be in its relaxed state at the same time - so the question arises: how is the aggregate relaxed state determined?

Take a simplified example, illustrated in Figure 4.5 and suppose that muscles A and B work in direct oppositions. That is, muscle A flexes a joint and muscle B extends the same joint. Muscle A will be in a relaxed state when it is not activated and therefore at its longest, or rest, length. Since muscles A and B are directly opposing each other, when muscle A is at its longest, muscle B will be at its shortest; therefore muscle B will be activated when muscle $\mathrm{A}$ is in a relaxed state. The opposite will be true for the relaxed state of muscle B. Therefore at the relaxed state of a joint, approximated by its mid-point, neither muscle can be in a fully relaxed state since neither muscle is at its rest length.

It is now evident why the following statement is misleading:

"When a muscle is at rest, it will often maintain a certain tonus to prevent the joint it controls from becoming too loose." [79]

First, as defined above, a muscle at rest has no tonus, or muscle tone, which is only generated with a contraction. Second, even in our simplified example where only two muscles control a joint's movement, if the muscle is relaxed the joint has an antagonist 


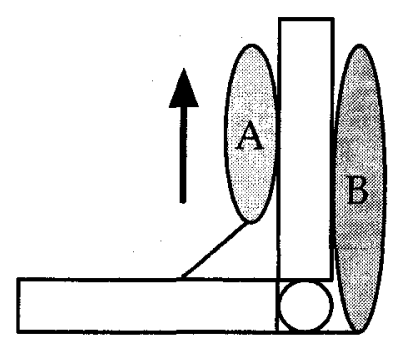

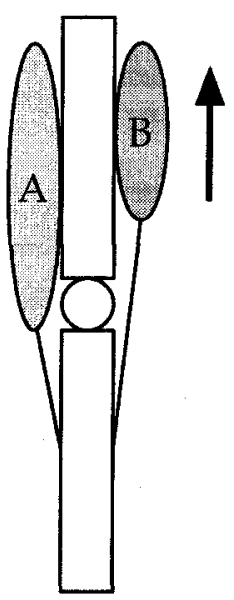

b

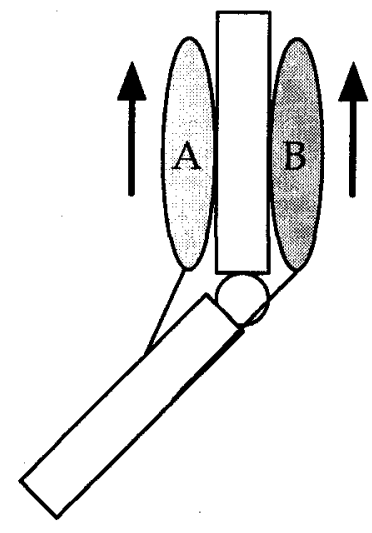

C

Figure 4.5: Two muscles act antagonistically, muscle A acts to flex the joint and muscle $\mathrm{B}$ acts to extend the joint. In (a) muscle $\mathrm{A}$ is contracted, muscle $\mathrm{B}$ is relaxed and the joint is fully flexed. In (b) muscle $B$ is contracted, muscle $A$ is relaxed and the joint is fully extended. In (c) the joint is relaxed and both muscle A and muscle B are active.

muscle controlling its position and it is not at risk of becoming 'too loose'. Finally, 'too loose' is ambiguous without some measure of what constitutes this level of joint instability.

The relaxed position of the joint is only able to determine the length of the muscle but not the force generated by the muscle. A muscle is not simply in a state of 'on' or 'off'; there is a range in the degree of activation of a muscle because each muscle fibre can be individually activated. At the same joint position, muscles can exhibit a range of degrees of activation since both muscles can be increasingly activated to work against the opposing muscle while still maintaining the same joint position.

For example, maintaining the posture of a flexed elbow requires little activation of the biceps and triceps muscles. When a bodybuilder maintains this posture during posing, the antagonistic muscles generate equal moments but create no motion at the joint. The larger muscle moments result in an increase in cross-sectional area of the muscles - an indication of increased muscular activation. As muscle fibres on either side of the joint are activated 
they produce force to match the resistance created by the antagonistic muscle; the force is proportional to cross-sectional area.

\subsection{Co-Contraction}

Co-contraction is the activation of more than one opposing muscle about a joint:

"Cocontraction (the simultaneous activation of antagonist muscles around a joint) provides the nervous system with a way to adapt the mechanical properties of the limb to changing task requirements - both in statics and during movement." $[73]$

\subsubsection{A Common Misconception Presented in Pedagogical Literature}

Piano pedagogues often refer to co-contraction, but every reference portrays cocontraction as a negative occurrence that is to be avoided and declares it as a cause of injury. Some examples of references to co-contraction are included below.

"The real goal of sensing the weight of your arm is to free its muscles from useless co-contraction." [79]

"Spreading the fingers (abduction) and playing simultaneously is co-contraction which makes it harder to play and can eventually lead to injury." [89]

"If I lift my fingers while maintaining the "curl" of the two end joints, I am using flexor muscles and extensor muscles simultaneously; in other words, I am co-contracting. Co-contraction is one cause of injury; there are pianists who have suffered injury from playing with curled fingers." [77]

In her approach, Taubman refers to co-contraction as a dual-muscular pull [54], a behaviour that is to be avoided.

Although references to co-contraction are generally negative, Fraser warns against superficial discussion of the concept. 
"Over-simplifying the whole question of co-contraction - relaxing into flaccidity for instance - can lead to more problems that it solves." [79]

\subsubsection{Co-contraction in Biomechanical Engineering}

The movement of a joint is a complex, involving many muscles whose actions are interdependent. No muscle acts alone in the movement of a joint, there are agonist muscles, antagonist muscles, fixator muscles and neutralizer, or synergist, muscles. The muscles from each of these groups are outlined below using the example of elbow flexion.

Agonists: The agonists are the muscles that perform the desired action, in this case flexion of the elbow. Brachioradialis and bicpes brachii are both agonists, although the contribution from biceps brachii is greatly diminished when the forearm is pronated.

Antagonists: The antagonists oppose the desired action, in this case antagonists are elbow extendors. Triceps brachii is the only antagonist in this example.

Fixators: The fixators stabilize proximal joints while distal joints are weight-bearing. The trapezius muscle stabilizes the shoulder at the scapula, allowing elbow flexion to occur against a load.

Neutralizer: The neutralizers resist the actions of undesired movements. If the elbow flexion is occuring with a pronated forearm, pronator quadratus and pronator teres resist forearm supination, which would naturally occur due to the role of the biceps brachii in supination.

The activities of the muscles differ based on the task; Jongen et al [90] found marked differences in distribution of activities of synergistic flexor muscles during co-contraction and flexion tasks. This suggests the existense of inhibitory mechanisms for antagonist muscles.

Not every movement will require co-contraction, but it is required in many instances. For example, in turning a doorknob both the biceps and triceps will be activated: the biceps for the supination action of turning the knob; and the triceps to resist elbow flexion, which 
naturally occurs during biceps contraction. In this case co-contraction exists synergetically, that is the antagonist is activated to aid with the desired movement by eliminating the effects of undesired movements. Another case in which co-contraction is required is for posture and orientation. Here, co-contraction would occur if it was desired to maintain a constant joint angle. Milner [91] found that stabilization of the hand is more effective using posture than joint stiffness, indicating the importance of co-contraction and posture.

Some recent research on co-contraction in the arm is summarized below.

In 1998 Gribble and Ostry [92] measured EMG activity in flexors and extensors of the shoulder and elbow. Subjects completed point-to-point motion trials with varied starting positions. Data showed that EMG levels are a function of the amplitude of motion and that they varied with the relative direction of rotation in the joints (similar or opposing rotation). This led to the hypothesis that muscle co-contraction may be a strategy to compensate for the introduction of forces in multijoint limb dynamics.

In 2001, Suzuki et al [93] measured muscular contractions using EMG and their results indicate that co-contraction is associated with phasic muscle activity. The level of activation varied with movement speed, suggesting that there is a simple control strategy to adjust co-contraction, which may be implemented prior to the beginning of the movement.

In 2003, Gribble et al [73] measured contractions using EMG and noted an inverse relationship between the degree of co-contraction and the size of the target in a point-to-point movement. From this, it is suggested that co-contraction may serve as a strategy for improving accuracy in multi-joint arm movements. Additionally, a decrease in the level of cocontraction decreased with the number of trials performed, suggesting that co-contraction is diminished with learning.

In cases when co-contraction is undesirable, motor control pathways induce reciprocal inhibition. The antagonist muscles are controlled via inhibitory interneurons from the 
spinal cord that actively disable muscular contraction during agonist contraction. These interneurons are controlled by the brain and by length sensors in the agonist muscles [94].

Reciprocal inhibition is an automatic response induced during agonist contraction. When the activation command for the agonist muscle descends to the muscle, it is coupled with an inhibition command travelling to the antagonist muscle; they are activated simultaneously, but in parallel during voluntary contractions [95]. Inhibition occurs in most healthy individuals at rest, but the degree of inhibition produced varies considerably from person to person [95].

\subsection{Multi-joint Issues}

In reality, the movement of a joint is even more complex than described in the previous sections because the activity at one joint also affects neighbouring joints, which extends the situation to a multi-joint problem. In the example of elbow flexion, the main complication is the biceps brachii which has origin points on the coracoid process, a bony protrusion on the scapula, and the supraglenoid tubercle of the scapula and its insertion point on the radius. This means that the muscle crosses both the shoulder and the elbow. Therefore any activity of the biceps brachii for flexing the elbow or supinating the forearm also either creates motion at the shoulder or requires stabilization or neutralization at the shoulder.

A further complexity is added to this because many tasks are dependent on the position (or other parameter) of an endpoint and so in response the motions at all the joints in the limb defer to the functional requirements of the limb as a whole [96]. Many tasks performed by limbs are specified in terms of the endpoint (e.g. positioning the hand during manipulation tasks), and motions at the joints are usually subservient to these whole-limb functional needs. [96]

\subsubsection{An Unrecognized Problem in Piano Pedagogy}

To date, studies in piano pedagogy have regarded only isolated sections of the body, especially fingers. The body is a complex system that it is integrated at many levels, 
changes in function of one joint or muscle may affect the function other joints or muscles.

"Unfortunately, qualitative studies with regard to motions used in piano playing have generally focused on motions so small and isolated from the integrated work done by the entire upper half of the body as to be completely inapplicable to practical piano technique." [43]

Pedagogues may be referring to multi-joint issues when mentioning 'unity'.

"Firm your wrists and fingers just enough to allow them to move as a unit with the forearms." [5]

"The fingers, hands and arms must be positioned so that they can move as a unit." [54]

\subsubsection{Extension of Biomechanical Concepts From Single-Joint to Multi- Joint}

An example of work that could benefit by expanding to include multi-joint issues is the finger modelling work from Harding et al $[9,42]$

"When tendon tensions and joint forces are decreased by optimization of finger positions as described above, corresponding increases in forces and tensions are not necessarily seen elsewhere in the finger. Thus, minimizing the tension in one tendon usually reduces tensions in other tendons and forces in related joints." [9]

Researchers should extend this work to see the effects of the optimized finger positions on the proximal side of the wrist, and even within the hand. The muscles that control the fingers are not located in the fingers themselves but in the hand and the forearm. The analysis should include the contracting muscles to create a more accurate account of the effects of the finger position optimization. In this case the flexor muscles of interest are flexor digitorum profundus, flexor pollicis longus and flexor digitorum superficialis, which all originate in the forearm, and flexor carpi ulnaris, palmaris longus and flexor carpi radialis, which all originate on the humerus in the upper arm. These last three therefore cross two 
joints to reach the hand, so any changes in their activity will affect both function at both the elbow and wrist.

\section{Force, Position and Stiffness}

The force and position of the hand are related to the joint motions and moments of the endpoint stiffness by the relationship:

$$
\mathbf{K}=\frac{d \mathbf{F}}{d \mathbf{p}}
$$

where:

$\mathrm{K}$ is the endpoint stiffness matrix;

$\mathrm{F}$ are the forces at the hand; and

$p$ is the position of the hand.

For planar motion, $\mathrm{F}$ and $\mathrm{p}$ are vectors with $\mathrm{x}$ - and $\mathrm{y}$-direction components and $\mathrm{K}$ is the following $2 \times 2$ matrix:

$$
\mathrm{K}=\left[\begin{array}{ll}
k_{x x} & k_{x y} \\
k_{y x} & k_{y y}
\end{array}\right]
$$

$\boldsymbol{k}_{\boldsymbol{x} \boldsymbol{x}}$ and $\boldsymbol{k}_{\boldsymbol{y} \boldsymbol{y}}$ are the single-joint stiffneses. For example, in a model with joints at the shoulder and elbow, $\boldsymbol{k}_{\boldsymbol{x} \boldsymbol{x}}$ is the elbow stiffness and $\boldsymbol{k}_{\boldsymbol{y} \boldsymbol{y}}$ is the shoulder stiffness. Single-joint stiffness is dependent on both the single- and the multi-joint muscles that cross each joint. The off-diagonal terms, $\boldsymbol{k}_{\boldsymbol{x} \boldsymbol{y}}$ and $\boldsymbol{k}_{\boldsymbol{y} \boldsymbol{x}}$, represent the cross-joint stiffness and represent the interaction between the joints. The cross-joint stiffness is dependent only on the multi-joint muscles that cross both joints.

If the system appears to be passive, $\boldsymbol{k}_{\boldsymbol{x} \boldsymbol{y}}$ and $\boldsymbol{k}_{\boldsymbol{y} \boldsymbol{x}}$ are equal and it is called a conservative system and is described by "spring-like" behaviour. 
A non-conservative system is more complex and includes both symmetric and antisymmetric stiffness components.

$$
\mathbf{K}=\mathbf{K}_{\mathbf{s}}+\mathbf{K}_{\mathbf{a}}
$$

The symmetric component is the stiffness of a conservative system and the antisymmetric component is given by:

$$
K_{\mathrm{a}}=\frac{1}{2}\left(k_{x y}-k_{y x}\right)\left[\begin{array}{cc}
0 & 1 \\
-1 & 0
\end{array}\right]
$$

The majority of studies concerning human stiffness properties have been performed on single joints. However, some multi-joint studies exist, due to the recognition of the limitations of single-joint research.

Gomi and Kawato [8] extended the manipulandum experiments to include a multi-joint arm and therefore determine multi-joint arm stiffness. The study found that transverse movements had greater stiffness properties than longitudinal movements.

Osu and Gomi [97] studied multi-joint arm stiffness using EMG as a stiffness identifier. The arm model was a two-link system incorporating six muscles about the shoulder and elbow. The study demonstrated that motion at the elbow joint is controlled by both singleand multi-joint muscles while motion at the shoulder is only controlled only by singlejoint muscles. This leads to the conclusion that single- and multi-joint muscles are not coupled but instead multi-joint muscles are regulated independently according to the task. Additionally, the study found that during movement, stiffness at a joint can be as low as the cross-joint stiffness but that in static situations the single-joint stiffness is always greater than the cross-joint stiffness. The cross-joint stiffness is the off-diagonal terms of the stiffness matrix; $\boldsymbol{k}_{\boldsymbol{x} \boldsymbol{y}}$ and $\boldsymbol{k}_{\boldsymbol{y} \boldsymbol{x}}$.

Perreault et al [71] have identified three major limitations of the methods of multi-joint studies. 
"First the steady-state stiffness estimates employed in most studies ignore the much larger dynamic stiffness components that can strongly resist transient external disturbances. Second, estimates of steady-state stiffness obtained using transient (step or ramp) perturbations require that the subjects 'do not intervene' in response to step or pulse changes in endpoint position for intervals several times longer than voluntary reaction times. Third, those who have examined dynamic endpoint properties $[7,8,98]$ have made a priori assumptions about the structure of the endpoint dynamics and then fit parameters to this structure. These models typically assume linear elastic and viscous components as well as inertial contributions at the endpoint. Although single joint estimates indicate that this may be a reasonable assumption for small perturbations during postural conditions, it is unlikely to hold during less constrained experimental conditions $[99,100] . "$ 


\section{Chapter 5}

\section{Development of an Experimental Approach}

Quantification of impedance at the wrist during piano playing has never been attempted. As reviewed in Chapter 3, measurements are normally taken at an endpoint, a situation which is unrealistic for a complex task such as piano playing. For these two reasons, the question of determining impedance during piano playing is ambitious and requires a novel approach. The focus of this work is to develop and validate a methodology to accomplish this task, and for the purpose of this thesis the results of the process are of secondary importance.

\subsection{Experiment Overview}

The subject enters the lab having completed no warm-up exercises. The subject is fitted with retro-reflective markers on their right upper limb. The subject is seated at the piano and asked to sight read the supplied music (see Appendix B) while force perturbations are applied to their right wrist. A quantitative description of the performance is captured by the Disklavier that collects MIDI data, a haptic device that collects position and force data and a motion capture device that collects position data. The subject will play the piece in its entirety. If the subject did not experience "stiffening" during playing (did not undergo a significant increase in impedance), they will play the piece through a second time with the same data collection as previously. 


\subsection{Apparatus}

The objective of the experiment is to detect changes in the driving point impedance at the wrist during piano playing. The driving point impedance of the right wrist can be measured by means of a perturbation force (as applied, for example by the PHANTOM@ haptics device $^{1}$ ). This means that at the wrist, the Phantom generates a downward perturbation (applies a known force) and measures the displacement of the wrist. The consistent force application allows each perturbation to be compared using its displacement as a local measure of the impedance at the wrist; the changes in position must be due to the applied force and are thus a representation of the impedance.

The measurements taken during piano playing will consist of:

1. Force and displacement at the wrist, as measured by the Phantom Omni, from which a measure of the driving point impedance can be extracted;

2. MIDI data, as measured by the Yamaha Disklavier ${ }^{2}$ grand piano, including key velocity (a measure of loudness), pitch (or note) accuracy and timing accuracy (note on and note off), from which a quantitative description of the playing can be made for various measured impedances; and

3. Motion analysis, as measured by the Polaris $\left(\right.$ system $^{3}$, from which the position of the key, first knuckle and the arm of the Phantom Omni are recorded.

The Phantom Omni ${ }^{\top M}$ device (Figure 5.1) is a robotic machine that can apply up to 3.3 $\mathrm{N}$ force and can measure the force feedback within a workspace $160 \mathrm{~mm}$ wide by $120 \mathrm{~mm}$ high by $70 \mathrm{~mm}$ deep. The device can generate stiffness of up to $2.31 \mathrm{~N} / \mathrm{mm}$ in the vertical axis and has six degree-of-freedom positional sensing. The device will be positioned on the wrist and generate a downward perturbation at the wrist during piano playing while simultaneously collecting force and position data.

\footnotetext{
${ }^{1}$ SensAble Technologies, Inc. Woburn, MA

${ }^{2}$ Yamaha Canada Music Ltd. Toronto, ON

${ }^{3}$ Northern Digital Inc. Waterloo, ON
} 


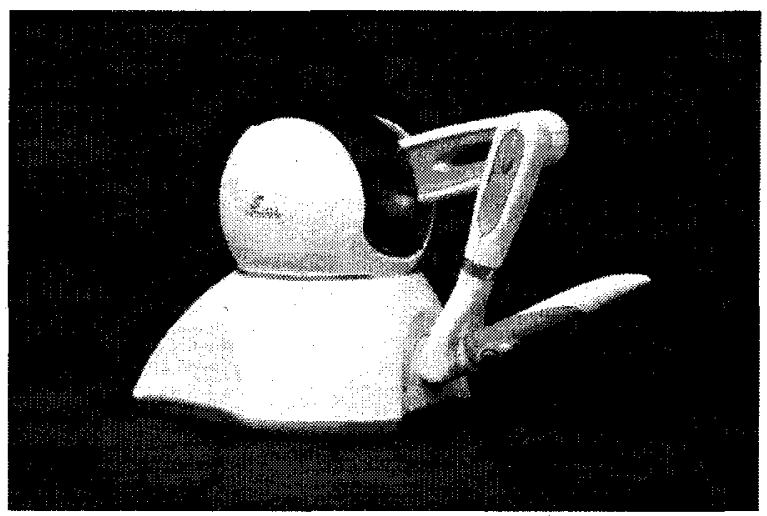

Figure 5.1: The Phantom Omni is a haptic device that can create and measure forces and displacements.

Table 5.1: Marker Definitions

\begin{tabular}{|l|l|}
\hline Number & Definition \\
\hline 1 & Piano key played by the right index finger \\
2 & Fingernail of the right index finger \\
4 & $\begin{array}{l}\text { Arm of the Phantom, near the stylus } \\
\text { Metacarpophalangeal (MCP) joint of the right index finger, at the head of the } \\
\end{array}$ \\
\hline
\end{tabular}

The position measured by the haptic device is at the intersection of the longitudinal axis of the stylus and the axis of rotation of the stylus.

The Polaris system (Figure 5.2) is a motion capture system that detects the positions of retro-reflective markers in three-dimensional space. Markers attached to the body allow the system to track the movements of the body in time and space. The Polaris is able to sample at a maximum rate of $30 \mathrm{~Hz}$.

Reflective markers for motion analysis will be placed on the fingernail, the metacarpophalangeal (MCP) joint (first knuckle), the piano key and the arm of the Phantom Omni, at the attachment site of the stylus, see Table 5.1 and Figure 5.3.

The Disklavier (Figure 5.4) grand piano looks and feels like an acoustic grand piano, having the same hammer mechanism. However, it has the additional feature of using optical 


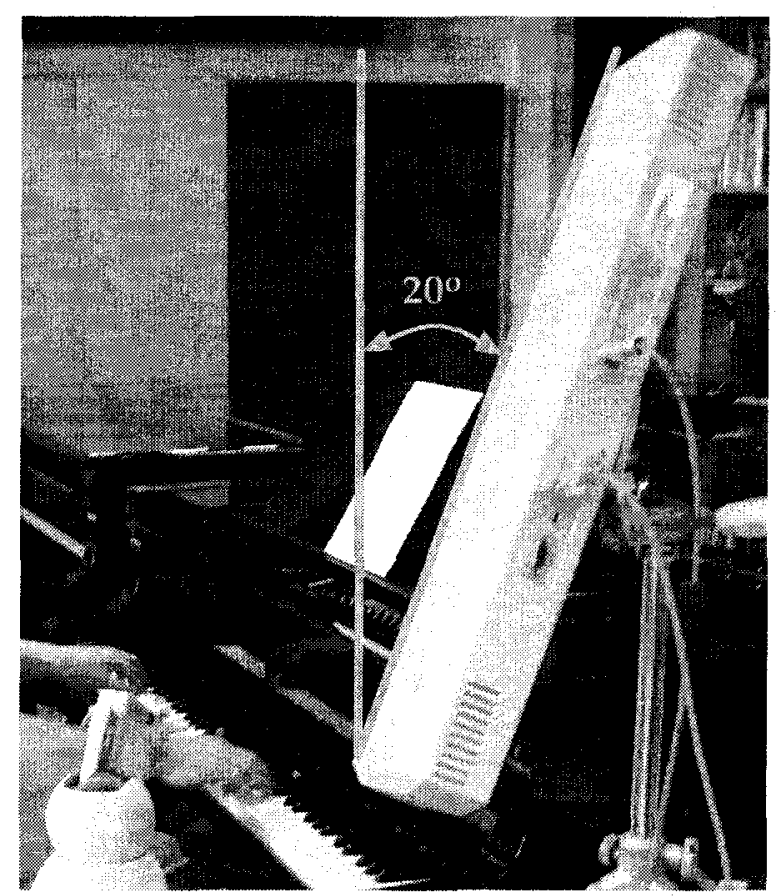

Figure 5.2: The Polaris system detects the position of retro-reflective markers in threedimensional space and time. In our experiment the system was rotated to optimize viewing of the markers.

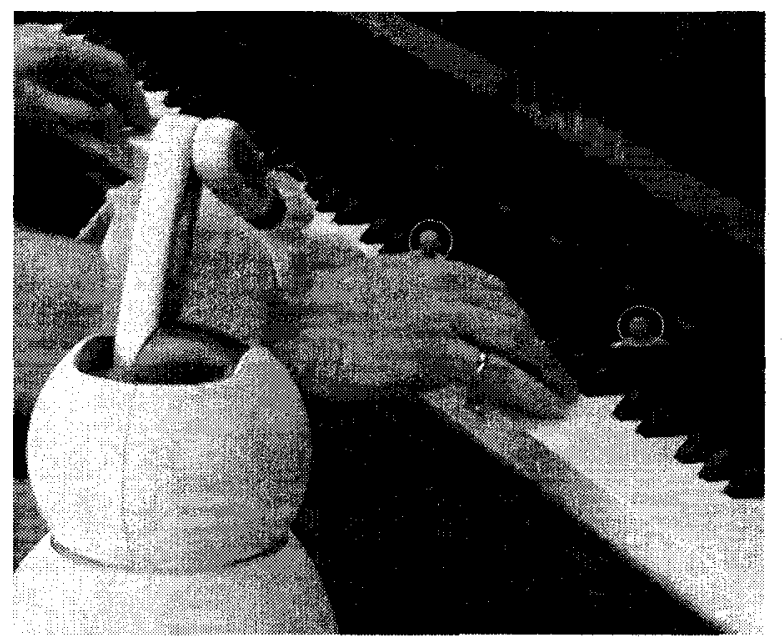

Figure 5.3: The markers on the subject, while playing, are indicated by the circles. A fourth marker on the fingernail of the index finger cannot be seen. 
sensors to monitor and record the performance, including note name, onset, velocity and release.

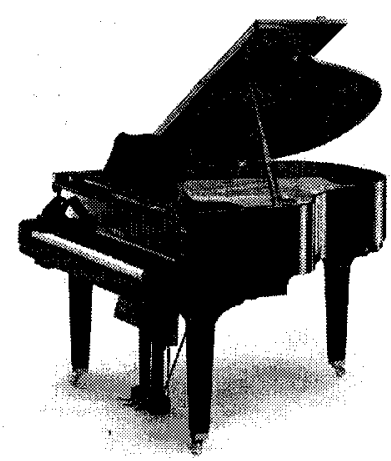

Figure 5.4: The Yamaha Disklavier grand piano plays like an acoustic piano but also has optical sensors to record the performance. The DGB1CD model is shown here.

The Phantom's stylus was attached to the subject's forearm just medial to the wrist to allow full flexion and extension of the wrist during playing. This was accomplished using a custom-made block (Appendix A) that was bandaged to the forearm, as shown in Figure 5.5. The bandage was wrapped around the forearm twice to cushion the subject from the block. The block was then held in place by wrapping the bandage around it and securing it to the forearm, aligned lengthwise with the arm.

\subsection{Methods}

\subsubsection{Task Description}

Independent variables have been selected to promote detectable changes in driving point impedance and allow for some music-related conclusions. The impedance is expected to differ among subjects, as well as for one subject with changes due to the duration of the performance, the tempo and the dynamics of the music played.

The subject sight-reads a short two-handed piece of music on a Disklavier grand piano in the Piano Pedagogy Research Laboratory at the University of Ottawa while measurements are taken. The music was composed by Ann Southam, a respected modern composer from Toronto, who wrote the piece to meet our requirements. The goal of the piece is to cause 


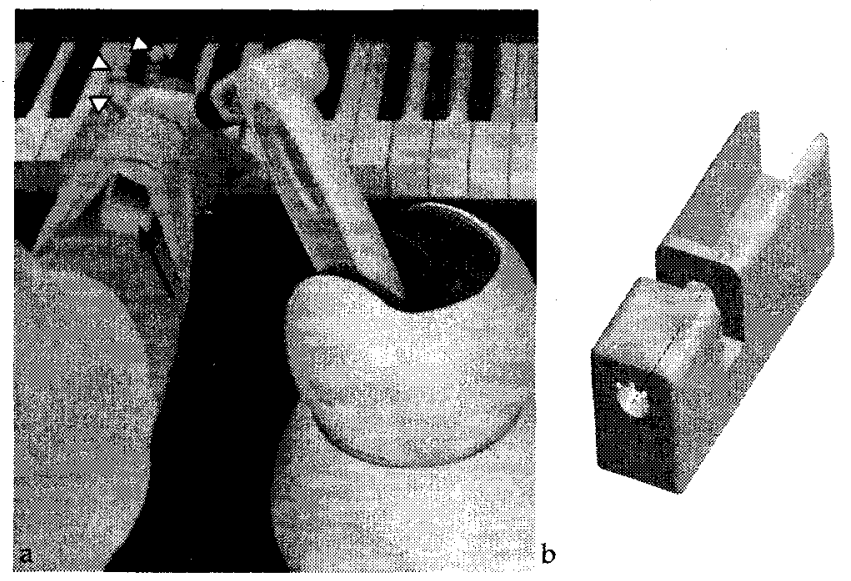

Figure 5.5: The Phantom was attached to the subject's forearm, just medial to the wrist, using a block that was bandaged to the forearm. In Figure ' $a$ ' the arrowheads indicate the markers on the key, the subject's first knuckle and the Phantom, and the arrow indicates the block that is secured to the arm with a bandage. Figure ' $b$ ' is a three-dimensional representation of the block used as a connector between the subject and the Phantom.

the player's wrist to undergo a significant increase in impedance after approximately one minute of playing, even in experienced pianists. There are several constraints placed on the piece based on the requirements of data collection and analysis.

The piece must require minimal lateral arm movement (constrained to 6 inches of lateral wrist movement, or roughly a 5-note span). The motion of the shoulder, elbow and wrist must be predominantly in the same vertical plane, to simplify subsequent calculations the right-hand index finger must play a note between a twelfth and two octaves above middle $\mathrm{C}$. The perturbation must be synchronized with the tempo. The right-hand index finger must always play the same note to allow for comparison of impedance measurements during playing; similarly, the left-hand index finger must play the same note in the event that measurements are taken using the left arm. Finally, the index finger must not play faster than a quarter note at $120 \mathrm{bpm}$, due to timing restrictions on the measurements arising from neural feedback.

The initial conceptions for the piece are: 
1. To begin at a slower tempo (using whole notes) and increase the tempo (decrease the note lengths) as the piece progresses;

2. To have a gradual crescendo throughout the piece, that is to increase the volume or loudness as the piece progresses;

3. The piece is largely unpredictable, to require mental concentration and deliberate finger movement; and

4. The piece contains repeated sections that allow comparison of playing at different tempi and dynamic levels.

The music Ann Southam composed in response to our description is found in Appendix B.

\subsubsection{Experimental Software}

The experiment will be controlled by software written by the author specifically for this purpose. The software has inputs from both the Disklavier and the Phantom and sends output to the Phantom to create the perturbation.

The musical instrument digital interface (MIDI) event data from the Disklavier is transferred to a computer that monitors that incoming data for the desired notes. When these events occur, the computer communicates with the Phantom, triggering a perturbation at the subject's wrist.

\section{Pseudo Code}

Below is the pseudo code for the experimental software, for more detail see code listing 1 in Appendix C.

Declare Global Variables Create pointers to files for MIDI and Phantom output, a start time for the test, handles to the MIDI server and the MIDI port and an array of integers that specifies which notes to trigger. Declare pointers to the callback code for the Phantom and the read process for the MIDI devices. 


\section{Main: Coordination of Communication}

Create Output Files Create unique file names encoded with the date and time and open files for both MIDI data and Phantom data. Print start times and headers to the files: Time, Position $(\mathrm{x}, \mathrm{y}, \mathrm{z})$ and Force in the Phantom file.

Initialize MIDI Communication Find the MIDI Devices and their inputs. Initialize client handles and create input ports. Return errors if handles or ports not created. Iterate through the MIDI inputs and connect to each.

Initialize the Phantom Device Initialize the Phantom Omni and return errors if it fails. Start the scheduler running. Call the callback code.

Sleep the Main Thread Sleep the main thread while the read process and the callback code run. For this music, 95 seconds is sufficient to record data for the piece.

Clean-up Close connections to the MIDI inputs, stop the scheduler and disable the Phantom.

\section{MyReadProc: MIDI Devices}

Handle the MIDI Data Create a counter for the number of G4 notes played, a flag for G4 activities, an elapsed time variable and a counter for the MIDI packets. Also create a variable to signal trigger notes to the Phantom. Collect packets of MIDI data from each input port. For the Disklavier the first bit is encoded for the activity performed, such as key press or key release; the second bit is the note played; and the third bit is the velocity.

Log Activity Print each packet to the MIDI output file along with the elapsed time since the start of the test.

Find G4 Key Presses For the Disklavier, bit $1=144$ indicates a key press and bit $2=$ $4 \mathrm{~F}$ indicates G4. Scan the data for this occurrence and flag the event when it occurs 
to ensure that only the first key press is recorded for any note played. Record the event in the MIDI output file.

Find Trigger Keys Compare the counter for the G4 notes to the current integer in the array. When equal, step forward to the next integer in the array, signal the Phantom that a trigger note has been played by altering the signalling variable. Record the event in the MIDI output file.

Find G4 Key Releases For the Disklavier, bit $1=80$ is a key release. Scan the data for a key release of $\mathrm{G} 4$ (bit $2=4 \mathrm{~F}$ ) and return the flag to normal to signal that the key has been released. Signal the Phantom that the trigger note has been released by returning the signalling variable to normal status. Record the event in the MIDI output file.

Repeat Iterate through to the next packet. If the elapsed time has surpassed 95 seconds, exit the routine.

\section{Callback Code: Phantom}

Set Up Specify the direction and amplitude of the force to apply. Create a flag to signal a change of state in force application and a variable for the elapsed time since the start of the test. Begin the frame of communication with the Phantom.

Apply the Force Scan the activation state of the variable in the MIDI read process. If it signals to apply the force, then disable force ramping and enable force output using the force vector specified above. Alter the flag status to indicate the force is on.

Remove the Force Scan the activation state of the variable in the MIDI read process. If it signals to remove the force and the flag indicates that the force is on, then disable the force output and change the flag status to indicate the force is off.

Record the Data Introduce a counter to record only every 50th data point. When the counter reaches 50 , record the elapsed time, the $\mathrm{x}, \mathrm{y}$ and $\mathrm{z}$ position and the current force in the Phantom file and then reset the counter to 0 . 
Table 5.2: Summary of similar perturbation studies

\begin{tabular}{cccccc}
\hline Paper & Subjects & $\begin{array}{c}\text { Duration of } \\
\text { perturbation } \\
(\mathrm{ms})\end{array}$ & $\begin{array}{c}\text { Distance of } \\
\text { perturbation } \\
(\mathrm{mm})\end{array}$ & $\begin{array}{c}\text { Force } \\
(\mathrm{N})\end{array}$ & Other \\
\hline Gomi, $1997[8]$ & - & 250 & - & - & 72 trials \\
Latash, $1994[101]$ & 4 & 2000 & torque & - & 4 min oscillation \\
Flash, $1990[6]$ & - & 1420 & 4 or 8 & 2 & 15 positions \\
Corcos, $2002[102]$ & 8 & - & "2 distances" & - & fatiguing protocol \\
McIntyre, $1996[103]$ & 3 & 1500 & $1-4$ & $0,2.5$ & various loads applied \\
Tsuji, 1995 [7] & - & 400 & peak of 5 & $+/-4$ & 8 directions \\
Zhang, 1997 [104] & 6 & 30,000 & - & - & stochastic pert \\
\hline
\end{tabular}

Clean Up End the frame of communication with the Phantom. If the elapsed time has surpassed 95 seconds, exit the routine. Check for errors during force rendering and print errors to screen. Return the Callback.

\subsubsection{Perturbation Description}

\section{Similar Studies}

Several papers outline their methods used to measure limb stiffness. From these, the number of subjects, the duration of the perturbations, the duration of the trial and the distance of the perturbations (they appear to be mostly distance-controlled rather than force-controlled) were extracted and summarized in Table 5.2.

Although these experiments offer a good starting point for our methodology, we are not repeating work previously completed. Our experiment adds a level of complexity on top of that studied in the publications cited. By measuring the impedance at a point within the limb (wrist), as opposed to the endpoint (fingertip), the mechanical system is different from previous experimental studies as a result of a reduction in the number of degrees of freedom in the system and the presence of multiple interactions. The limb is interacting with the environment at two points: the point of the driving point impedance measurement, and the point of contact with the piano keys. 


\section{Perturbation Definition}

There is a general agreement that a voluntary response is not active until after $400 \mathrm{~ms}$, and that small amplitude perturbations reduce the effects of the stretch reflex. Therefore it is suggested to start with a short perturbation duration of less than $400 \mathrm{~ms}$, and an amplitude of $3.0 \mathrm{~N}$. The duration of the perturbation will place limits on the tempo of the task because the perturbation should only occur while a key is depressed. A constraint made on the piece is that the measured quarter notes must be no faster than $120 \mathrm{bpm}$, or the notes will have at least a 500-ms duration. This will allow for some error in the timing of the perturbation while still having it occur during the bottom of the keystroke.

The music provides a range of note lengths to analyze. The tempo is set to a quarter note at $120 \mathrm{bpm}$, meaning that each half note should last $1000 \mathrm{~ms}$, each quarter note 500 $\mathrm{ms}$ and each triplet $333 \mathrm{~ms}$. The perturbation length will be tied to the note length, and therefore perturbations will last from $333 \mathrm{~ms}$ to $1 \mathrm{sec}$, if the subject plays at tempo.

The force amplitude will be set to $3.0 \mathrm{~N}$. If a perturbation occurs while the key is not depressed, this low amplitude force perturbation will not cause a finger to depress the key.

A simple biomechanical analysis supports this claim. Assuming a body weight of $70 \mathrm{~kg}$ and a limb segment length of $40 \mathrm{~cm}(l)$ from fingertip to elbow, the example shown in Figure 5.6 can be analyzed. The assumption is made that the finger must only support the weight of the hand and the forearm, but not that of the upper arm. Anthropometric data [105] estimates the mass of such a limb segment as $1.54 \mathrm{~kg}(\mathrm{~m})$ and the centre of mass as acting at $12.72 \mathrm{~cm}(x)$ from the proximal end of the segment (at the elbow). From here a moment balance demonstrates that the finger must push with $5 \mathrm{~N}(\mathrm{~F})$ to overcome the gravitational force of the arm's mass acting at the centre of mass. This is evidence that a force amplitude of $3.0 \mathrm{~N}$ for the perturbation will not be sufficient to depress the key. 


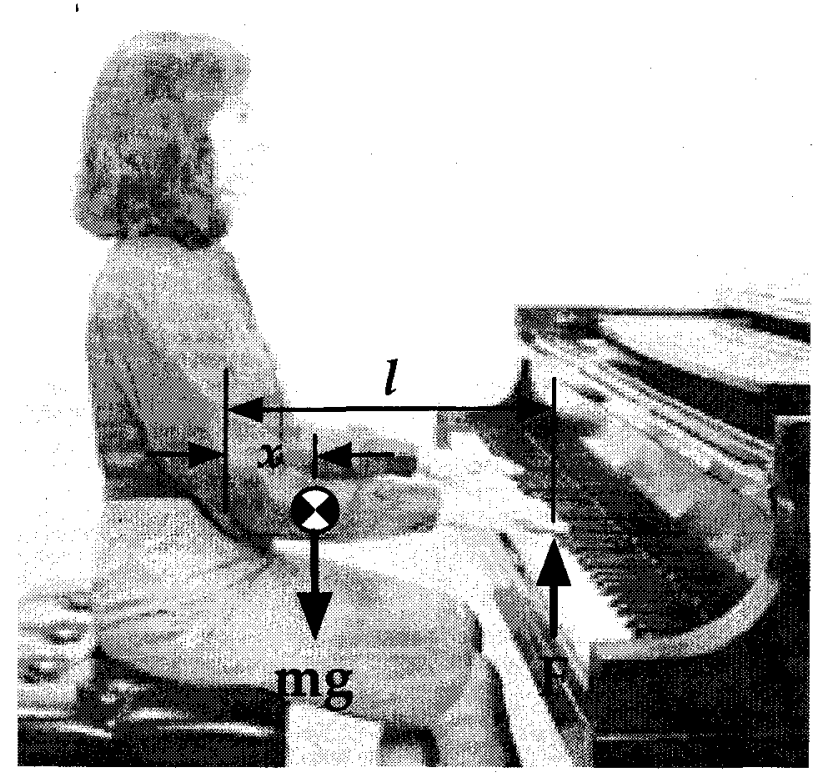

Figure 5.6: The free body diagram of the forearm and hand at the piano. A moment balance about the elbow allows the calculation of force, F, when values are established for the length to the centre of mass, $x$; the total segment length, $l$; and segment mass, $\mathrm{m}$. 


\section{Chapter 6}

\section{Results}

\section{$6.1 \quad$ Subjects}

Two initial subjects underwent experimental testing as described in the previous chapter. To maintain anonymity, the subjects will be referred to as subject 1 and subject 2 .

Both subjects had prior knowledge of the tests to be performed, although subject 2 was more familiar with the experiment. They are both experienced piano players associated with the Piano Pedagogy Research Laboratory at the University of Ottawa. The task of sight-reading the piece was not overly challenging for either player and minimal errors were recorded. These errors occurred mostly in bars 17-20, where both subjects commented that the random nature of the music was a challenge to sight-read and play accurately. Both subjects commented following the experiment that they did not experience an increased impedance at the wrist due to their performance; they did not feel 'stiffer'.

Both subjects chose to play at a tempo slower than that set in the piece (120 bpm to a quarter note, or $500 \mathrm{~ms}$ per quarter note). The tempo selected by each was only slightly slower than the set tempo, but was not steady throughout the piece.

Position data of subject 1 and subject 2 are shown below in Figure 6.1 and Figure 6.2, respectively. It is apparent that subject 1 used more wrist movement in their playing and the 3-dimensional graph shows a rocking motion of the wrist. The wrist motion of both subjects occurs in all three dimensions. The reactions to the perturbations appear in the 
plot as the many deviations that occur in the y-direction.

Another view of the three-dimensions of the data is to look at each with respect to time, as shown in Figures 6.3 and 6.4 for subjects 1 and 2 respectively. In this view it is evident that the perturbations' effects occur in the $\mathrm{y}$-direction. The $\mathrm{x}$ - and z-direction data have negligible responses to the perturbations and are excluded from further study.

\subsection{Polaris}

Two devices, the Phantom and the Polaris, measured and recorded 3-dimensional position data for a location approximately on the subject's right wrist. The results of the Polaris data are found in Figure 6.5 for subject 1 and Figure 6.6 for subject 2 .

A comparison of the two data sets, shown at approximately the same angle, indicate that the Polaris did not sample as frequently as the Phantom. The Polaris data will be excluded from further study because of the low sampling rate of the position data.

\subsection{Phantom}

The wrist position measured by the Phantom was used for the analysis of the results, but it was not measured at a position directly on the wrist. The Phantom measured the position at the intersection of the longitudinal axis of the stylus and the rotational axis of the stylus.

To ensure the viability of using this measure as wrist position, measurements were taken of the position during perturbations while the Phantom was connected to a subject's arm and the arm at rest on a table. The results of these tests, Figure 6.7 shows the maximum displacement, show that the change in vertical position due to soft tissue compression is no more than $1.5 \mathrm{~mm}$. The average of the five measurements is $1.34 \mathrm{~mm}$. In comparison to the perturbations, this is assumed to be negligible. 


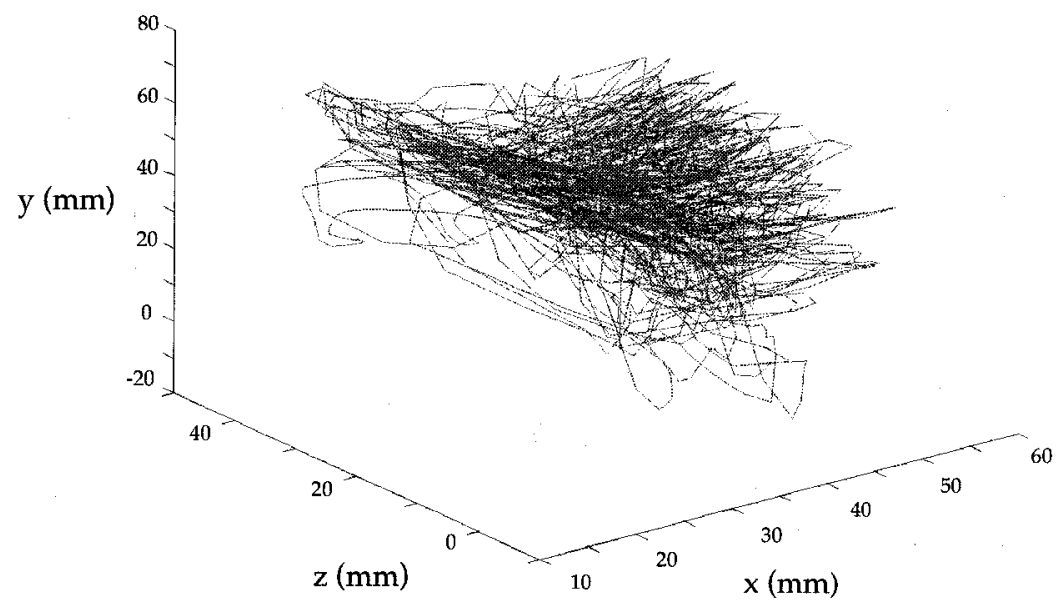

Figure 6.1: The position data of subject 1 measured by the Phantom, plotted in 3 dimensions.

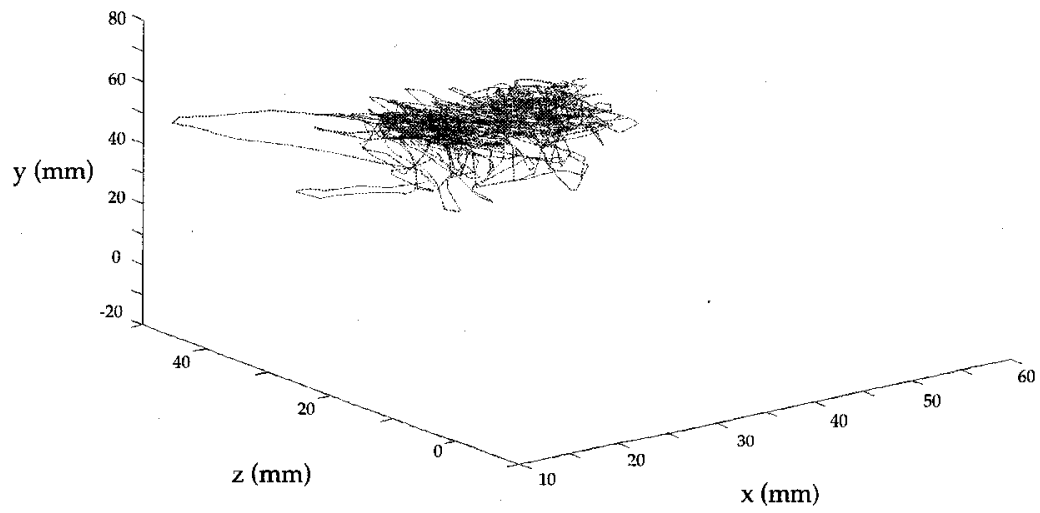

Figure 6.2: The position data of subject 2 measured by the Phantom, plotted in 3 dimensions. 


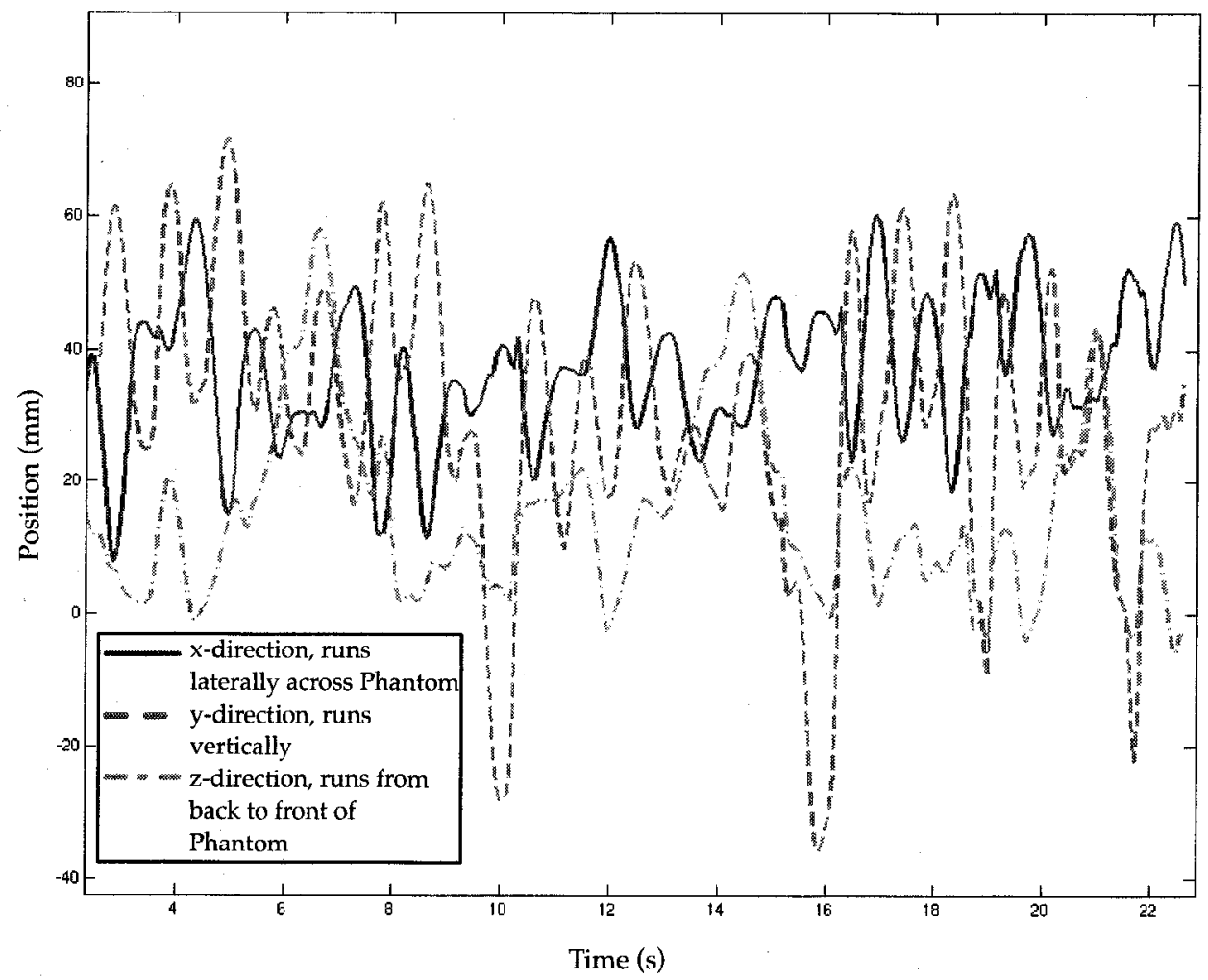

Figure 6.3: Plot of each dimensional response to the perturbation with respect to time, for subject 1 , reading 1 , bars 22-25. The perturbations are evident in the $y$-direction at approximately 10, 16, 19 and 22 seconds. 


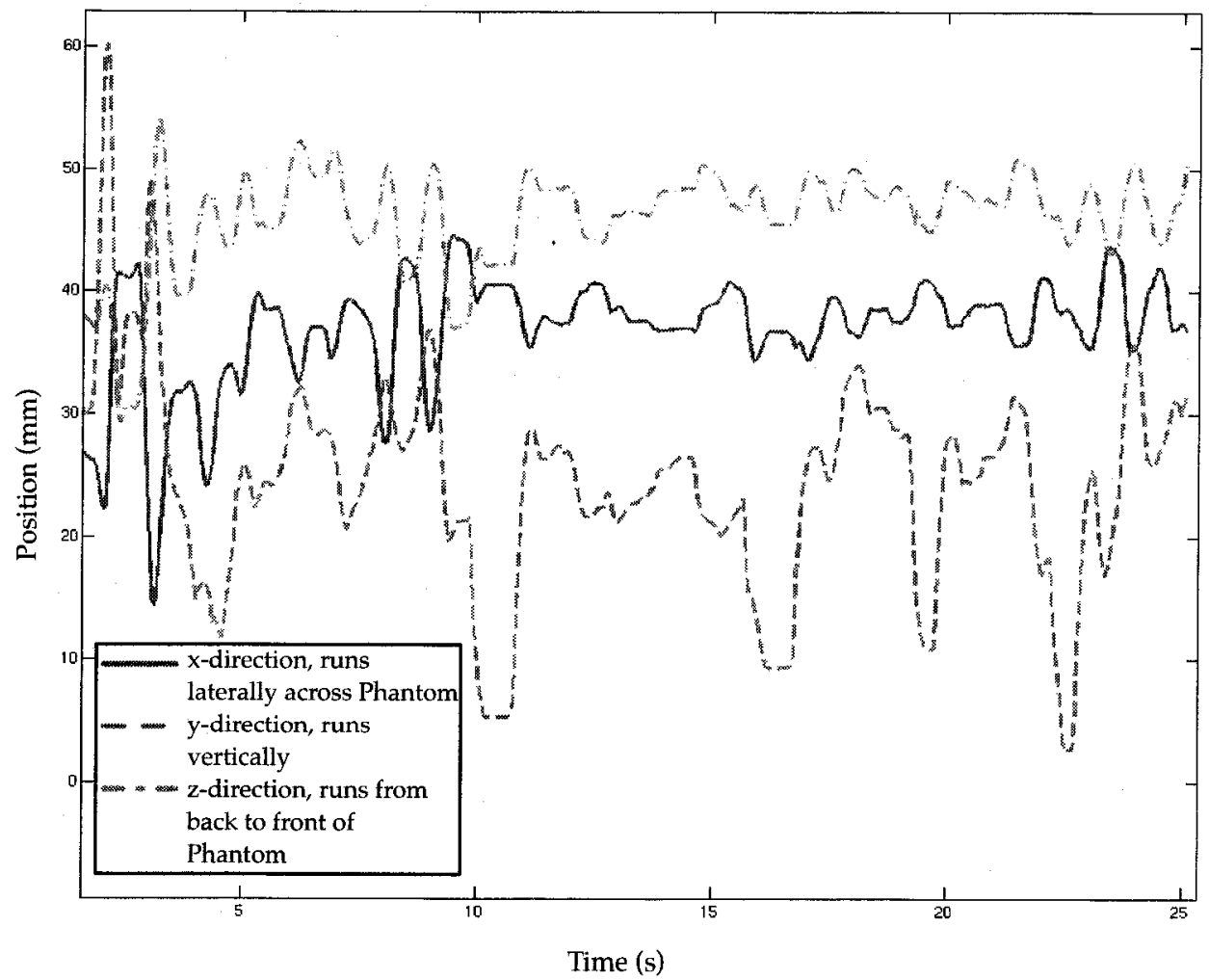

Figure 6.4: Plot of each dimensional response to the perturbation with respect to time, for subject 2 , reading 1 , bars 1-4. The perturbations are evident in the y-direction at approximately 10, 16, 20 and 22 seconds. 


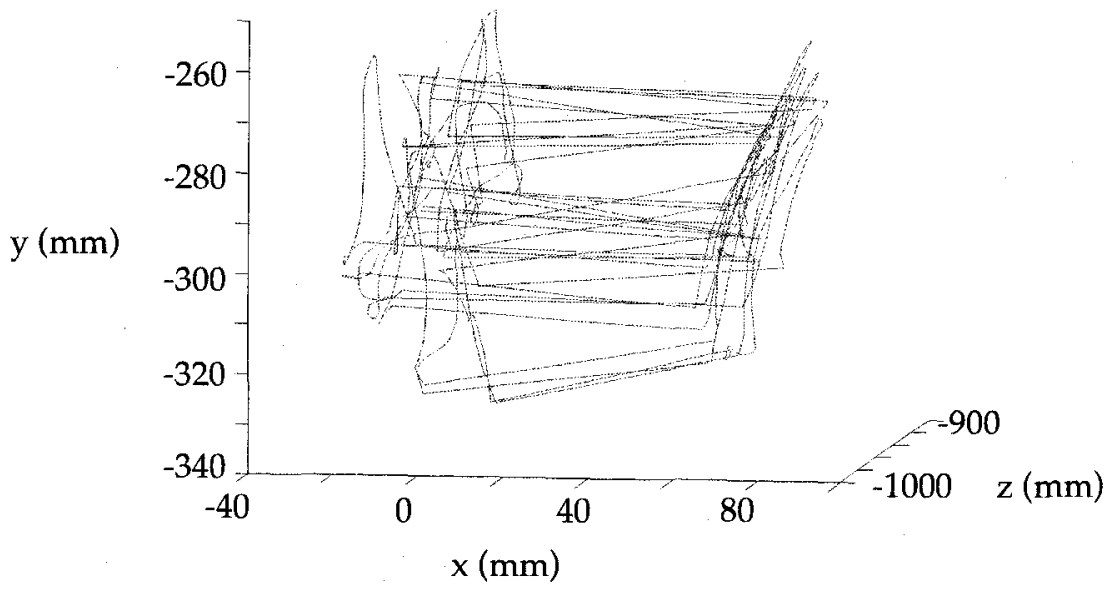

Figure 6.5: The position data of subject 1 measured by the Polaris, plotted in 3 dimensions. The coordinate system is relative to the Polaris, but the figure has been rotated to show the same view as Figure 6.1 of the Phantom data.

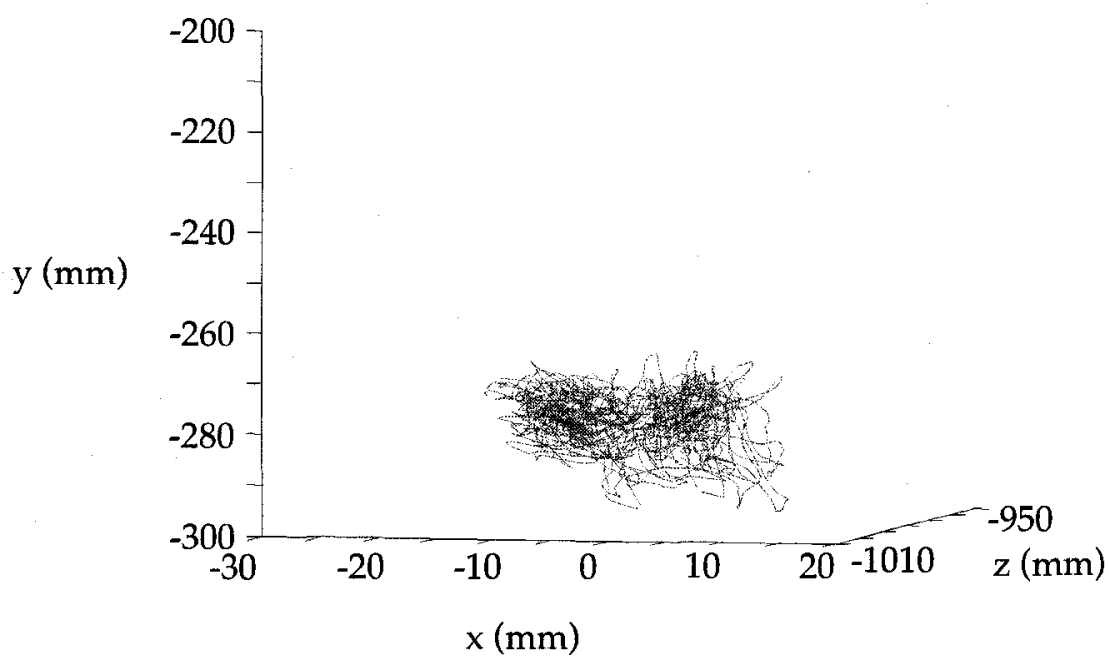

Figure 6.6: The position data of subject 2 measured by the Polaris, plotted in 3 dimensions. The coordinate system is relative to the Polaris, but the figure has been rotated to show the same view as Figure 6.2 of the Phantom data. 


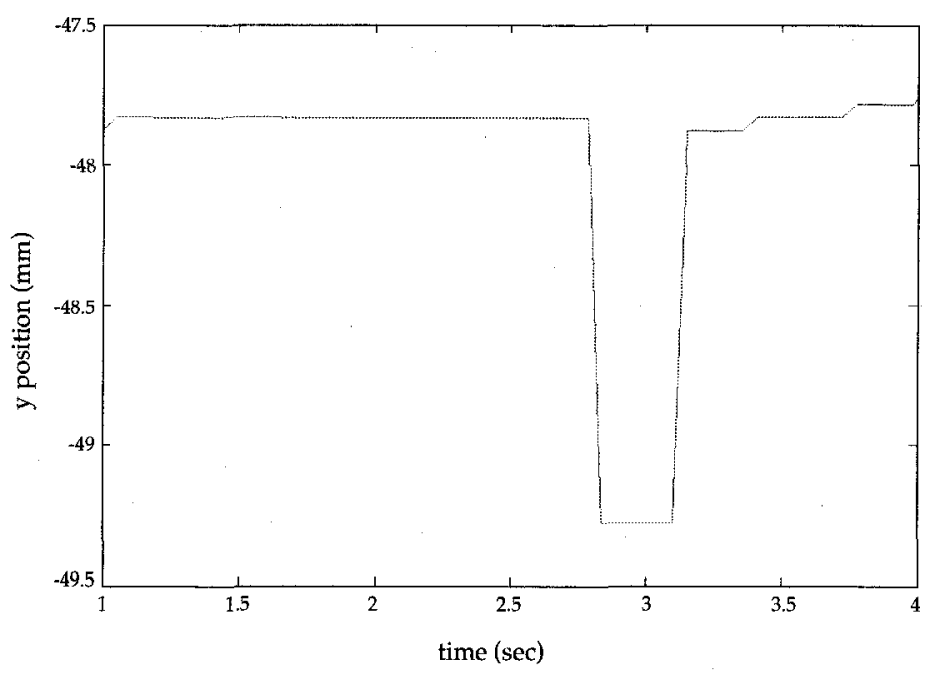

Figure 6.7: Vertical position data of a perturbation on an arm at rest on a solid surface. The displacement is a measure of soft tissue compression on the arm.

\subsection{Perturbations}

There are 6 perturbations that occur in the music that are of value to analyze. All occur within the first 8 bars when the tempo is slow enough to view the entire reaction to the perturbation.

The first two analyzed perturbations are triggered by half notes (two beats per note) and the third and fourth are triggered by quarter notes (one beat per note). These occur at the beginning of the piece, in bars 1-4, and are repeated again at the end of the piece, in bars 22-25. And so each occurs twice during the piece. These are shown in Figures 6.8 and 6.9 for subject 1 and Figures 6.10 and 6.11 for subject 2 .

The last two perturbations, perturbations 5 and 6 , occur in bar 5 and are triggered by triplets (three notes per two beats). These are repeated in bars 6, 7 and 8 , and therefore each occurs four times during the piece. These are shown in Figures 6.12 and 6.13 for subject 1 and Figures 6.14 and 6.15 for subject 2 . 


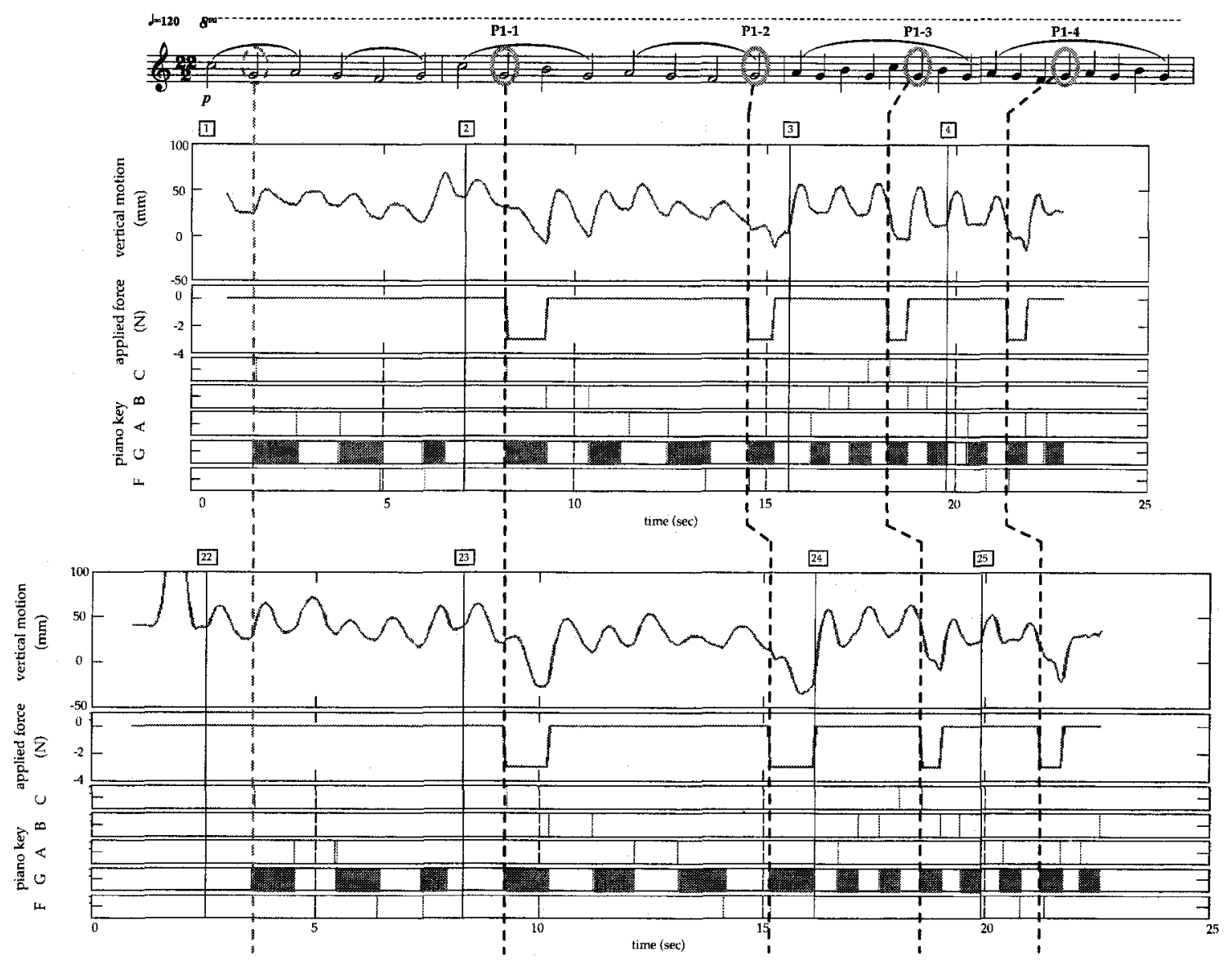

Figure 6.8: Perturbations 1-4, subject 1, reading 1. Data is normalized for equal spacing between the first two highlighted $G$ notes. Passage is played at the beginning and the end of the piece. Graph represents passage played, vertical position of the wrist and MIDI data, all aligned on the same time scale. Perturbations are indicated using solid ovals.

The graphs show the y-position of the wrist motion, the force applied by the Phantom, and the MIDI signals for each note (F,G,A,B and C). The MIDI signals for G have been filled in so that the solid rectangles indicate when the note is being played. Within the section of music, there are various marked notes. The markings are the following:

- the solid circles labelled 'PX-X' indicates a perturbation;

- the dashed circle labelled 'time shift' in bars 5-8 indicates the note compared to perturbation 5 in the time shift algorithm; and

- the dashed circle on the first $G$ in each passage indicates the note that was used 


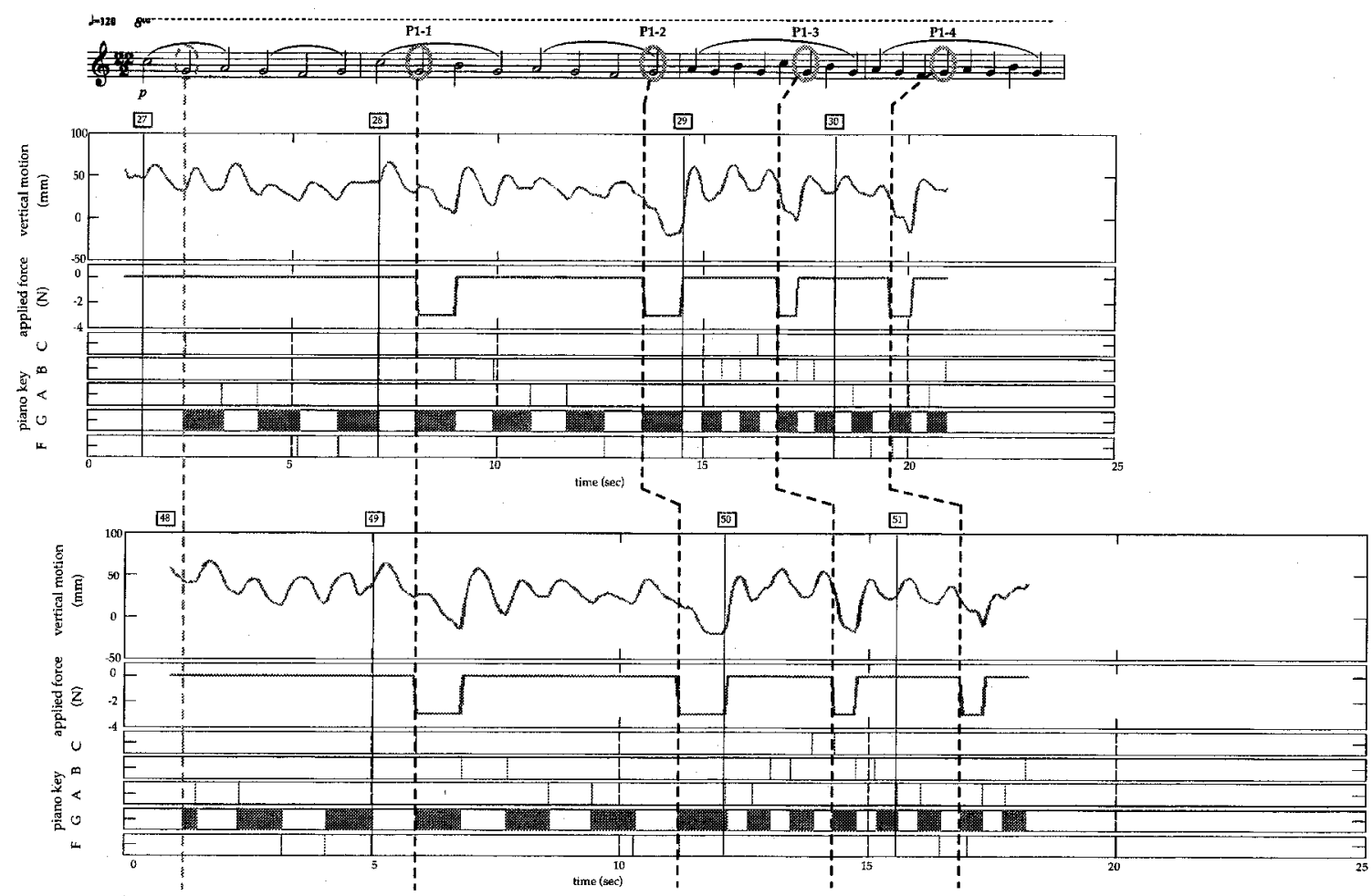

Figure 6.9: Perturbations 1-4, subject 1, reading 2. For details, see the caption of Figure 6.8 .

to align the data, the graphs were also normalized between this note and the first perturbation of each passage (either P1 or P5).

The graphs were aligned to minimize distortions due to tempo differences between trials.

The differences between the playing styles of the two subjects is obvious when comparing the vertical wrist motion shown in the Figures $6.8-6.15$. The rocking motion of subject 1 is similar to a oscillating waveform while the more restrained motion of subject 2 is a fairly flat line. In both cases, the perturbations can be seen easily through by visual inspection.

Mann-Whitney U Test statistical analysis (Appendix D) was performed to determine if the perturbations were significantly different from the unperturbed values. With $95 \%$ confidence $(\boldsymbol{p}<\mathbf{0 . 0 5})$ the vertical position at the end of every perturbation was significantly different from that of similar unperturbed notes. With $99 \%$ confidence $(\boldsymbol{p}<\mathbf{0 . 0 1})$ the vertical position at the end of every perturbation but P6 for subject 2 was significantly different 


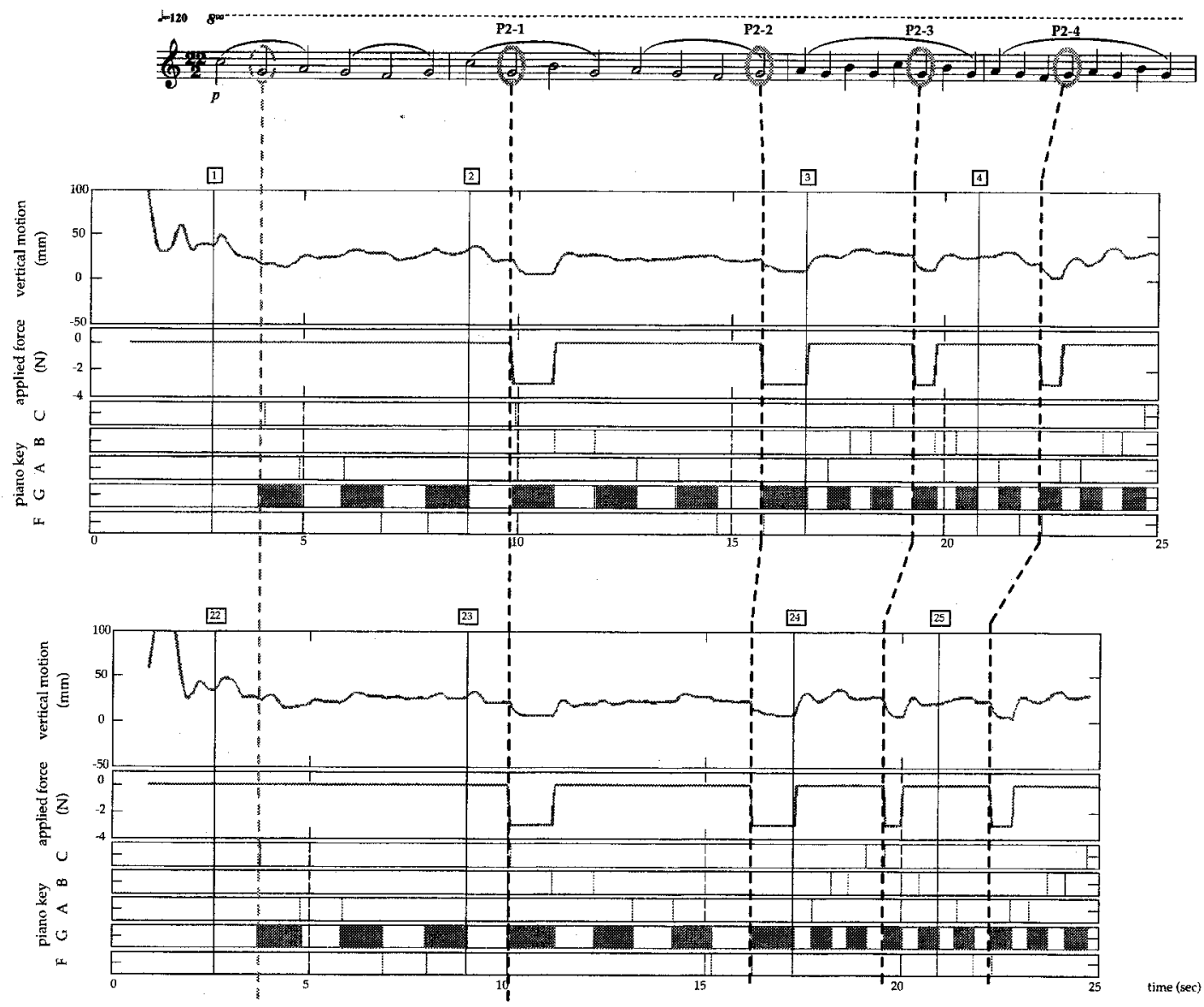

Figure 6.10: Perturbations 1-4, subject 2, reading 1. For details, see the caption of Figure 6.8 .

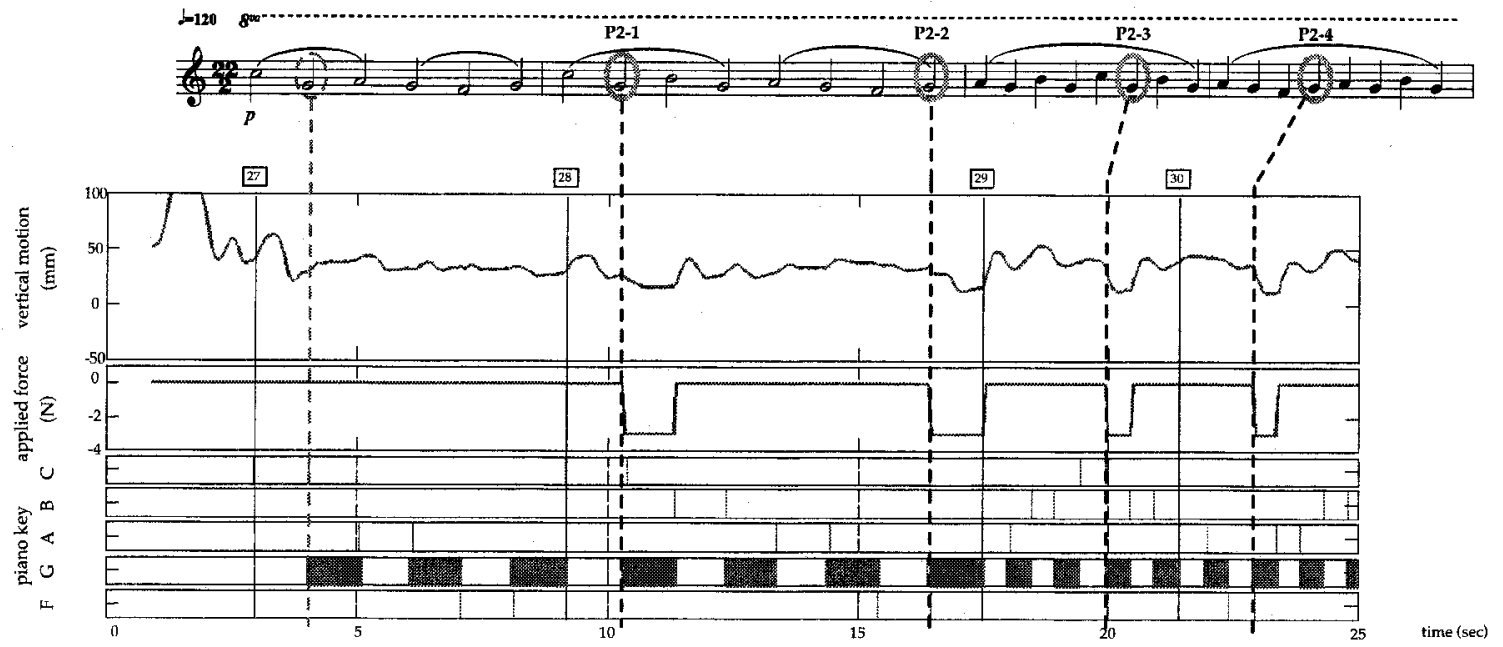

Figure 6.11: Perturbations 1-4, subject 2, reading 2. For details, see the caption of Figure 6.8 . 


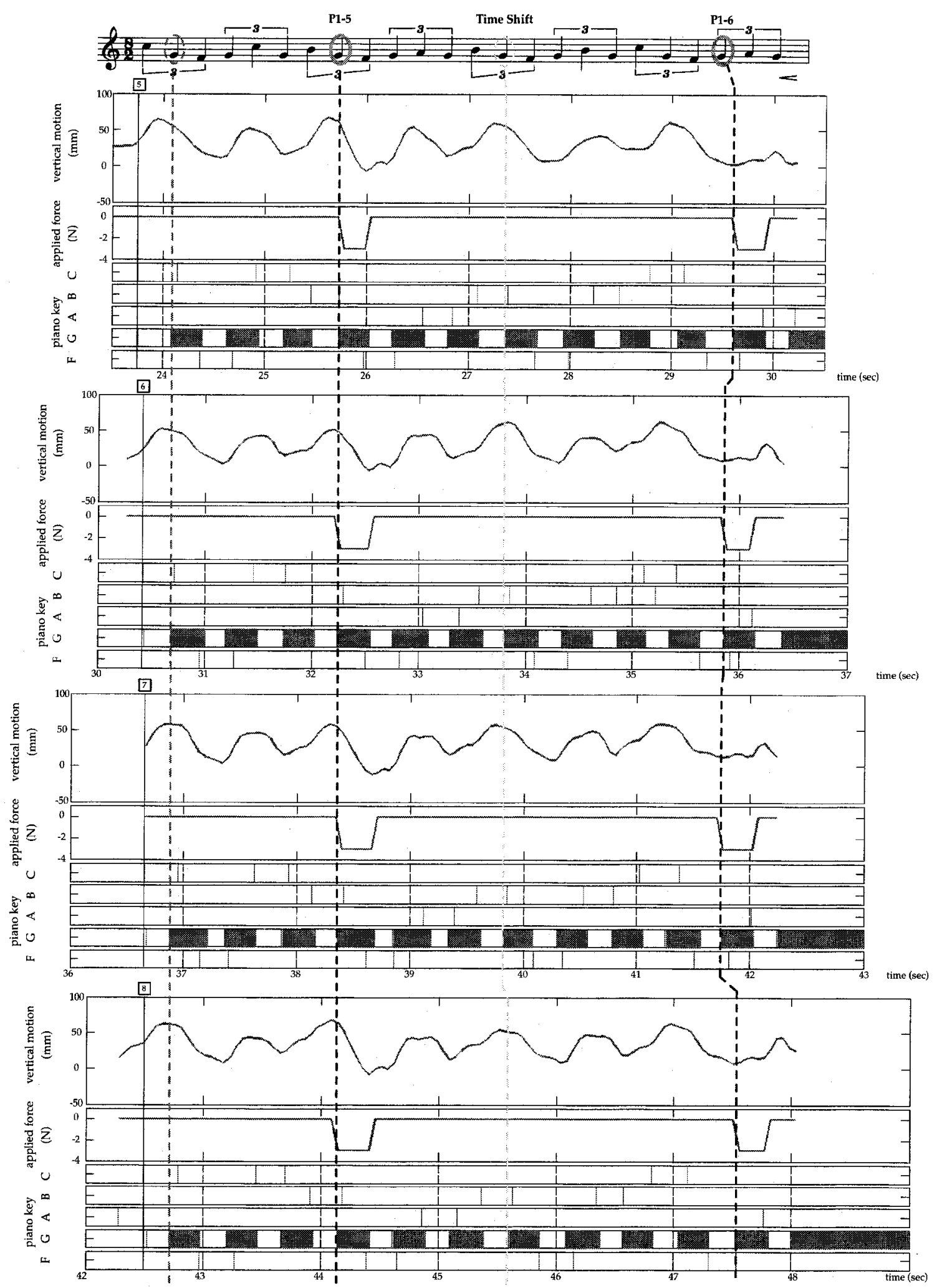

Figure 6.12: Perturbations 5-6, subject 1, reading 1. For details, see the caption of Figure 6.8 . 


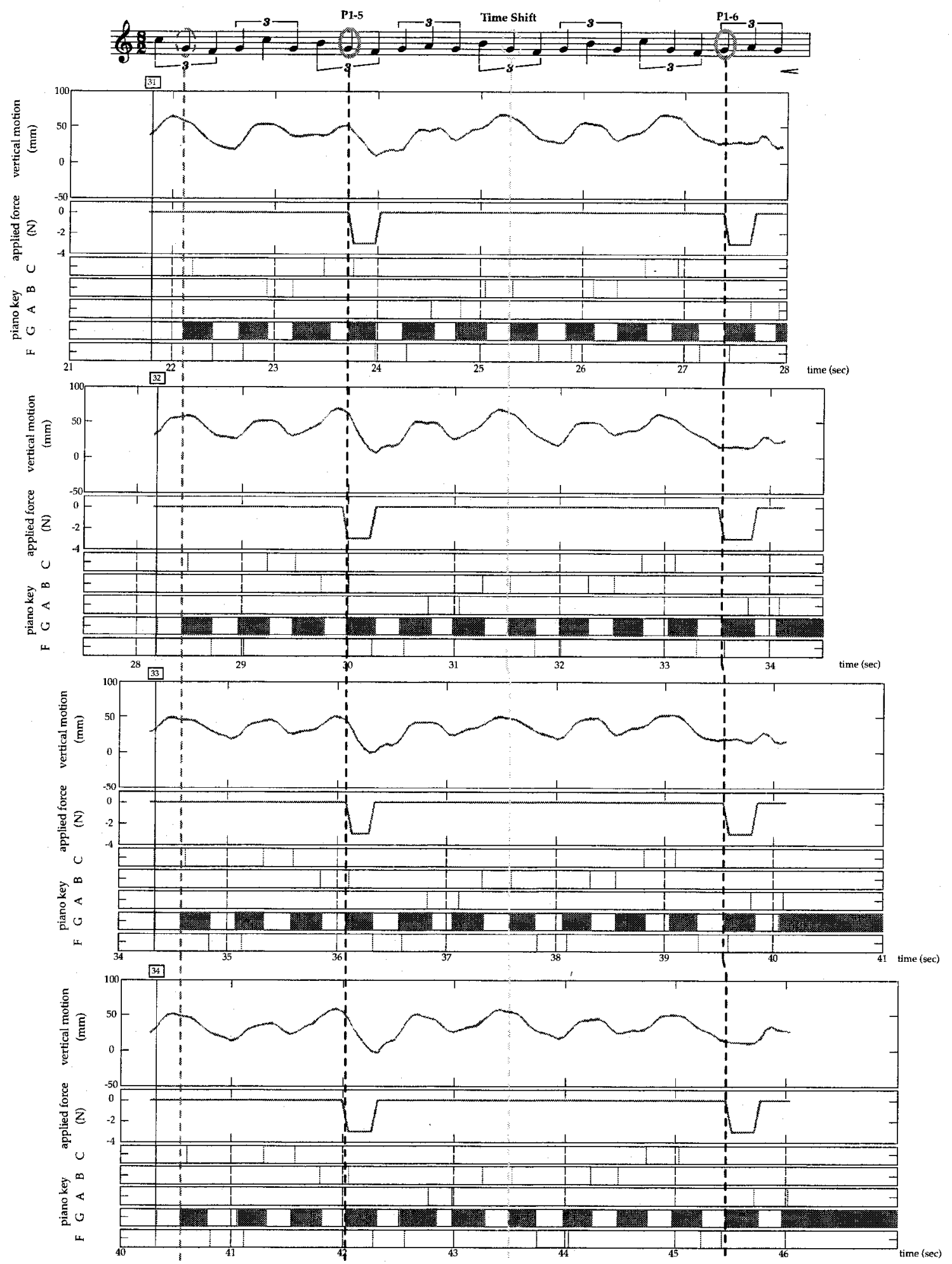

Figure 6.13: Perturbations 5-6, subject 1, reading 2. For details, see the caption of Figure 6.8 . 




Figure 6.14: Perturbations 5-6, subject 2, reading 1. For details, see the caption of Figure 6.8 . 


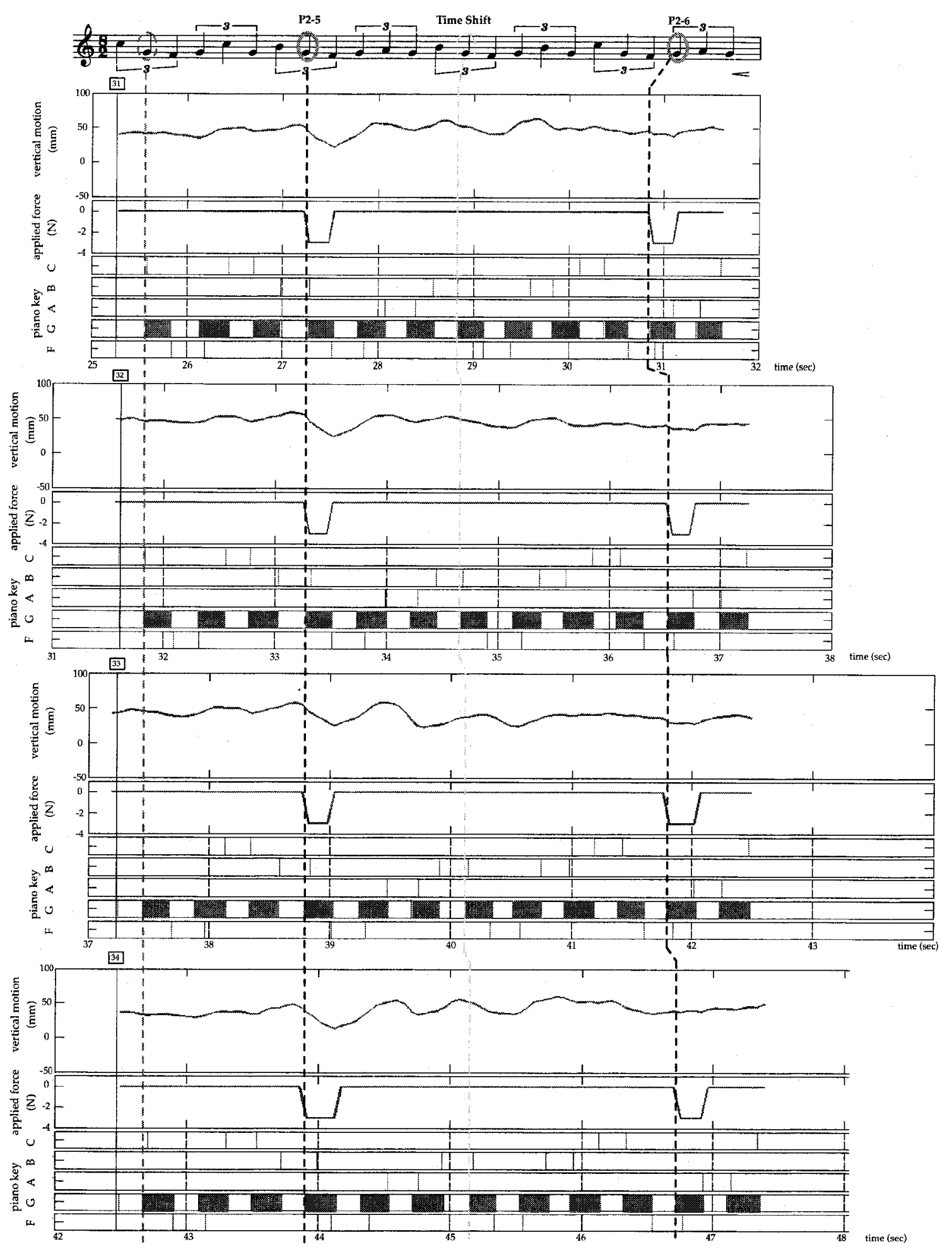

Figure 6.15: Perturbations 5-6, subject 2, reading 2. For details, see the caption of Figure 6.8 . 
from similar unperturbed notes. The analysis assumed stationary statistical properties.

Similarity of the unperturbed notes to the perturbed notes was determined as being the same note (G4) played for the same duration and at the same location within the grouping (i.e. first, middle, or last). In bars 1-4, only half notes were compared; the half notes were not grouped during playing for either subject so every occurrence of the same note (G4) can be compared. The half notes were compared using both the change in wrist position due to perturbation and the actual wrist position at note release. The fourth and seventh G4s were perturbed, their similar counterparts are the third, fifth and sixth G's of the piece.

In bars $5-8$, the notes were grouped into threes by subject 1 . Therefore the location within the grouping became important for similarity and the notes could only be compared using the wrist position at note release. The 19 th $\mathrm{G}$, referred to above as P5, is compared to occurrence number 16, 22 and 25 of G4 in the piece; each of these is played for the same duration and is the middle note in a grouping of three. The 26 th $\mathrm{G}$, referred to previously as $\mathrm{P} 6$, is compared to occurrence number 17, 20 and 23 of $\mathrm{G} 4$ in the piece; each of these is played for the same duration and is the first note in a grouping of three. 


\section{Chapter 7}

\section{Data Interpretation}

The results presented thus far only allow observation that perturbations occurred and created responses that were statistically different from unperturbed responses. Further analysis of the results allows for quantitative comparisons between the responses to the perturbations. In this chapter numerous algorithms are presented that each interpret the data in a different manner and calculate a perturbation height for each perturbation.

A challenging aspect of this goal is that although only two subjects were tested, the data from the subjects is quite dissimilar. Subject 1 has an evident rocking motion of the wrist during playing, which creates an oscillatory pattern in the y-direction position data. Subject 2 has a fairly still wrist during playing, which creates a fairly flat y-position plot. To meet this challenge, the data was analyzed using numerous interpretations.

The following algorithms were developed and used to calculate perturbation heights: basic onset, basic max, time shift and symmetry. The basic algorithms can be used for any case as they simply compare two heights. The time shift requires a repeating section that occurs in both a perturbed and an unperturbed state, in this case it is only applicable to perturbation 5 in bars 5-8. The symmetry algorithm approximates the response as an oscillation, and is therefore only applicable to subjects whose results fit this criteria. 


\subsection{Algorithms Based on Peak Heights}

The basic algorithm computes the height difference between maximum and minimum points. The maximum point is either the peak of waveform prior to perturbation onset (basic $\max$ ), or the point at the perturbation onset (basic onset). The minimum point is the lowest point reached during perturbation, or the valley.
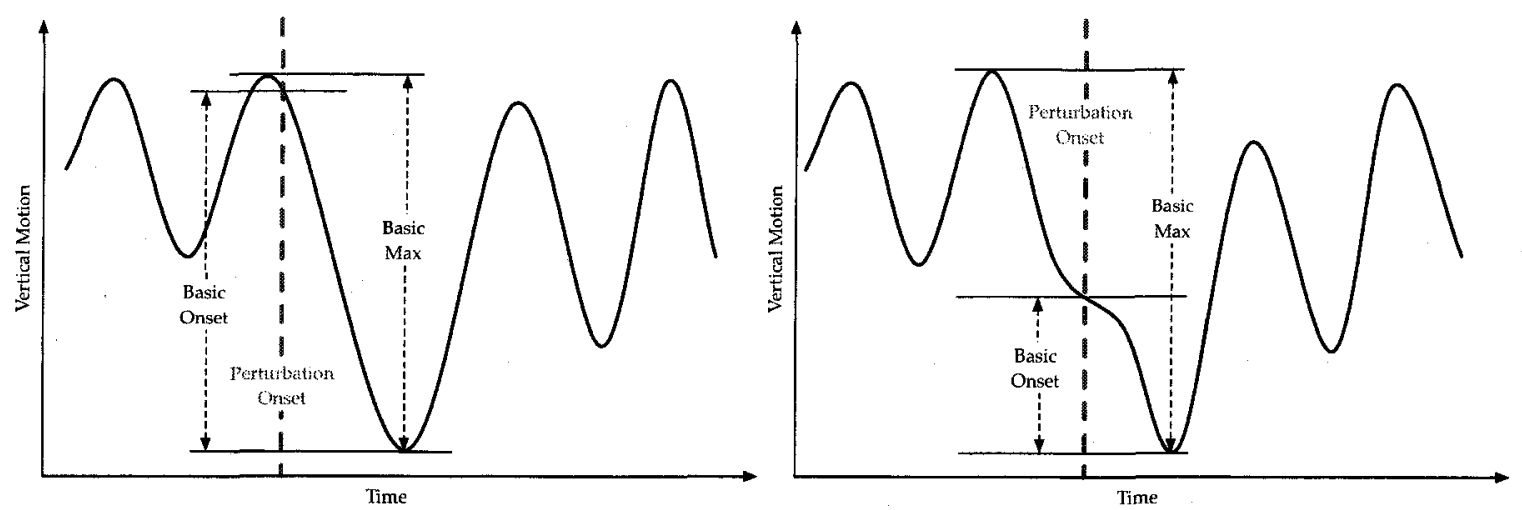

Figure 7.1: Graphical version of basic algorithms for two typical curves that approximate different aspects of the data. The basic onset algorithm calculates the difference in height of the wrist at perturbation onset and at the minimum. The basic max algorithm calculates the difference in height of the wrist at the maximum and minimum points. Notice that the values of the perturbation heights can vary significantly depending on when the perturbation onset occurs during the oscillation.

\subsubsection{Pseudo Code}

Below is the pseudo code for the basic algorithm, for more detail see code listing $2-1$ in Appendix E.

Load Data Load the Phantom dataset, containing y-position (height) and time. Load the MIDI dataset, containing perturbation onset and perturbation name. The perturbation onset is taken to coincide with 'note on' signal of the perturbed note.

Find the Perturbation Onset Cycle through the times in the Phantom dataset to find the time corresponding to the perturbation onset. Use linear interpolation between two data points surrounding the perturbation onset if the exact time does not exist in the Phantom dataset. Find the time of onset and the vertical position (height) at 
onset.

Find the Minimum Height Continue to cycle forward from the perturbation onset. Cycle through the heights in the Phantom dataset to find the local minimum height. Do this by comparing the height of each data point to the height of the following data point. When the following data point is greater than the current data point, you have found the minimum.

Find the Maximum Height Return to the perturbation onset. Cycle backwards through the heights in the Phantom dataset to find the local maximum height. Do this by comparing the height of each data point to the height of the following data point (before it in the iteration). When the following data point is smaller than the current data point, you have found the maximum. Find the maximum height and the time at which it occurs.

Calculate the Perturbation Height Calculate the Basic Max Algorithm as the difference between the local maximum and local minimum heights. Calculate the Basic Onset Algorithm as the difference between the height at perturbation onset and the local minimum height.

Iterate through the Perturbations Iterate through the MIDI dataset to the next perturbation time and repeat.

\subsection{Algorithms Based on Time-Shifting Unperturbed Sec- tions}

The time shift algorithm computes height difference between maximum and minimum points for two datasets, see Figure 7.2. The maximum point is the peak of the waveform prior to perturbation onset and the minimum point is the lowest point reached during perturbation, or the valley. The two datasets are perturbed and unperturbed. The string of notes G-B-G-F-G occurs twice in each of bars 5, 6, 7 and 8. The first occurrence in each bar is perturbation 5 and the second is an unperturbed string, as shown in Figure 7.3. The 
height difference of the unperturbed case is subtracted from the height difference of the perturbed case to give the calculated perturbation height.

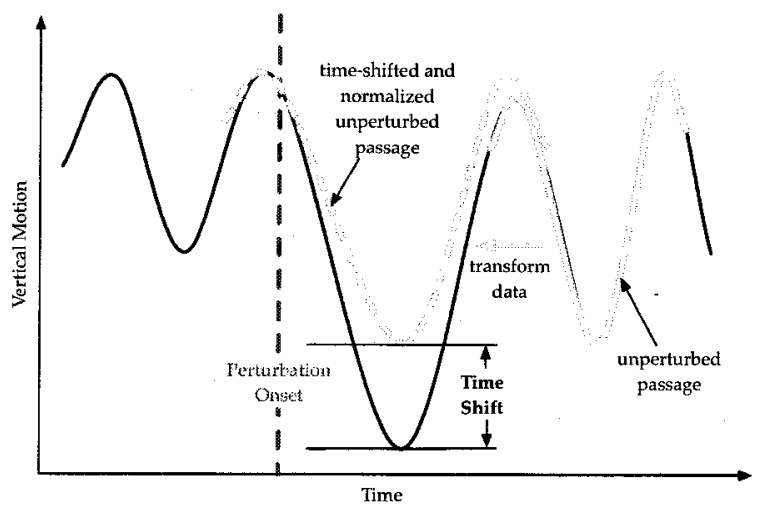

Figure 7.2: Graphical version of the time shift algorithm. The solid line is a representation of the data. The solid hollow line is the section of data representing the unperturbed passage and the dashed hollow line is the time-shifting of this data. The time shift algorithm calculates the difference between the maximum and minimum points in the perturbed data and in the unperturbed, time-shifted data.

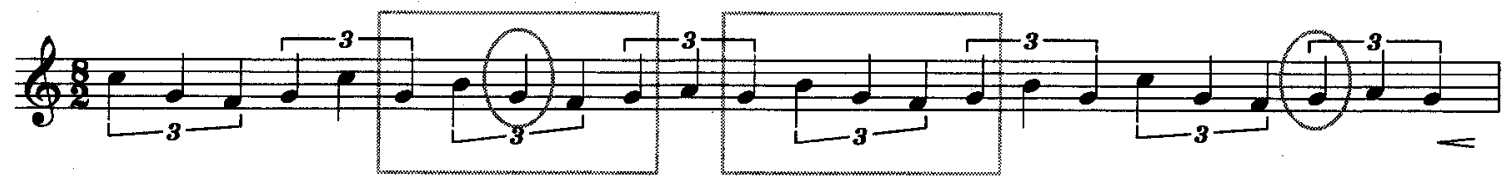

Figure 7.3: This is bar 5, where the time shift algorithm is applied. The box indicates the repeated section and the oval represents the perturbations that occur in the bar.

\subsubsection{Data Preparation}

Isolate the desired string of notes (G-B-G-F-G) for each bar (5-8) from Phantom dataset and for each tone (F, G, B) from MIDI dataset. The string appears twice in each bar, isolate the two cases separately. The first occurrence is the perturbed case and the second is the unperturbed case.

Time Shift Shift the times in each dataset to begin at zero by subtracting the initial time from each data point. Perform this separately for the two cases.

Normalize Find the two height peaks in each case. Divide the times at which the first peak occurs in the perturbed case by the time at which the first peak occurs in 
the unperturbed case. Similarly for the second peak. Average the two quotients to estimate the percentage difference between the frequency of the two waveforms. Use this value to normalize the shifted times of the unperturbed dataset. That is, multiply the modified times in the unperturbed data (from the time shift step) by the average percentage difference. This gives the normalized times of the unperturbed data.

\subsubsection{Pseudo Code}

Below is the pseudo code for the time shift algorithm, for more detail see code listing 2-2 in Appendix F.

Load Data Load the perturbed Phantom dataset, containing y-position (height) and shifted time. Load the unperturbed Phantom dataset, containing y-position (height) and shifted and normalized times. Load the MIDI dataset, containing 'note on' and 'note off' signal times. The perturbation onset is taken to coincide with the 'note on' signal of the perturbed note. This is entered manually into the code for each case.

Find the Maximum Height in the Perturbed Data Assign initial maximum and minimum heights as the height of the first data point. Cycle through the times in the perturbed Phantom dataset to find the local maximum height. Compare the height of each data point to the assigned maximum height. If the value is greater than the assigned maximum, reassign the maximum to the current height. Stop when the perturbation onset time is reached.

Find the Minimum Height in the Perturbed Data Cycle through the times from perturbation onset onward to find the local minimum. Compare the height of the each data point to assigned minimum. If the value is less than the assigned minimum, reassign the minimum to the current height.

Find the Maximum Height in the Unperturbed Data Assign initial maximum and minimum heights as the height of the first data point. Cycle through the times in the unperturbed Phantom dataset to find the local maximum height. Compare the height of each data point to the assigned maximum height. If the value is greater than the 
assigned maximum, reassign the maximum to the current height. Stop when the time of the 'note on' signal of the middle G is reached; this is the time comparable to the perturbation onset.

Find the Minimum Height in the Unperturbed Data Continue to cycle through the times from stop point onward to find the local minimum. Compare the height of the each data point to assigned minimum. If the value is less than the assigned minimum, reassign the minimum to the current height.

Calculate the Perturbation Height Calculate the perturbed height as the difference between the perturbed maximum and perturbed minimum heights. Calculate the unperturbed height as the difference between the unperturbed maximum and unperturbed minimum heights. Calculate the perturbation height as difference between the perturbed height and the unperturbed height.

Iterate through the Perturbations Iterate through the MIDI dataset to the next perturbation time and repeat.

\subsection{Algorithm Based on Symmetrical Attributes of Oscilla- tory Response}

The Matlab code for the symmetry algorithm computes the height difference between maximum heights of two curves, as shown in Figure 7.4. One curve is the data recorded during a perturbation, the solid line in the figure. The other curve is extrapolated from the data, it is represented by the hollow curve in the figure. The extrapolation is determined based on the assumption of symmetrical oscillations in the wrist motion.

Working backwards from the time at which the minimum height of the perturbed curve occurs, the algorithm looks for the greatest slope of the curve. This is found by searching for the maximum velocity in the derivative of the data. In this case, the slope is negative and so the maximum velocity desired is actually the maximum negative velocity, or the minimum velocity. The extrapolation is then based on these two points, the minimum height and the 
minimum velocity, with the assumption that symmetry occurs about the point of minimum velocity.

The peak of the extrapolated curve is an estimation of where the peak should be had the curve not been perturbed. The perturbation height is taken as the difference between the actual maximum height and the peak of the extrapolated curve.

Note that in left-hand graph of Figure 7.4 the extrapolated symmetry height is higher than the actual height. This results in a negative perturbation height.
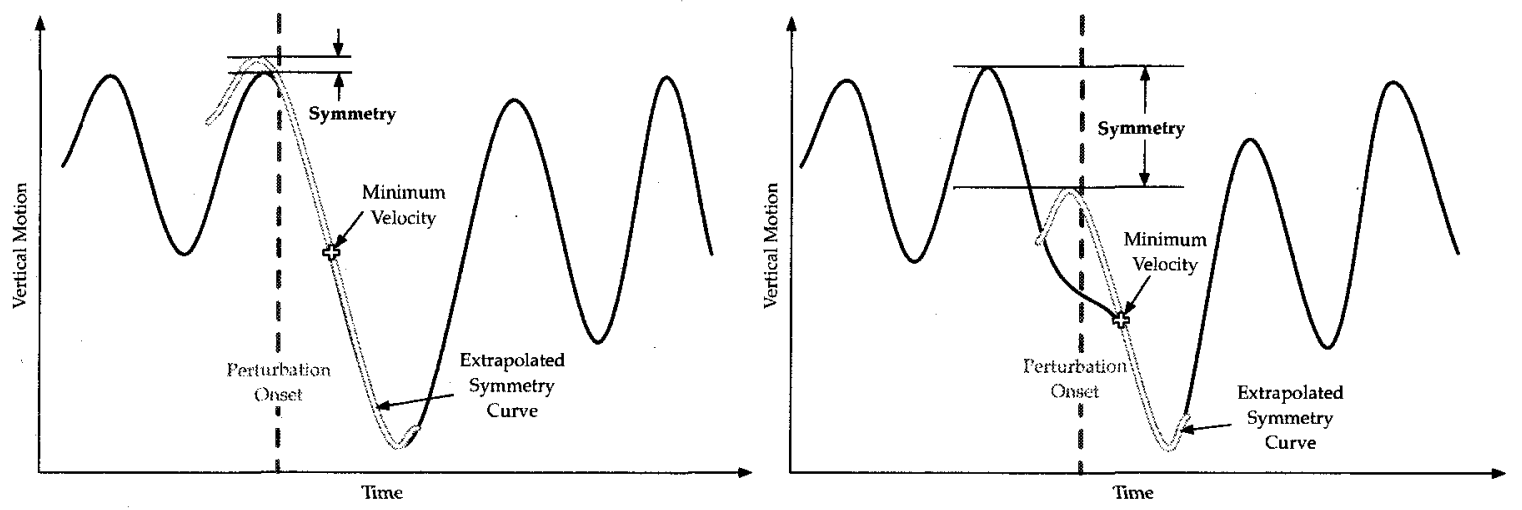

Figure 7.4: Graphical version of symmetry algorithm for two typical curves that approximate different aspects of the data. Create an extrapolated symmetry curve based on the minimum height and the point of minimum velocity. The perturbation height is then calculated as the difference between the actual maximum height and the extrapolated maximum height.

\subsubsection{Pseudo Code}

Below is the pseudo code for the symmetry algorithm, for more detail see code listing 2-3 in Appendix G. The code for the basic algorithm is also within the algorithm code lisiting.

Load Data Load the Phantom dataset, containing y-position (height) and time. Load the MIDI dataset, containing perturbation onset and perturbation name. The perturbation onset is taken to coincide with 'note on' signal of the perturbed note.

Find the Perturbation Onset Cycle through the times in the Phantom dataset to find the time corresponding to the perturbation onset. Use linear interpolation between 
two data points surrounding the perturbation onset if the exact time does not exist in the Phantom dataset. Find the time of onset and the vertical position (height) at onset.

Find the Minimum Height Continue to cycle forward from the perturbation onset. $\mathrm{Cy}$ cle through the heights in the Phantom dataset to find the local minimum height. Do this by comparing the height of each data point to the height of the following data point. When the following data point is greater than the current data point, it is the minimum.

Find the Maximum Height Return to the perturbation onset. Cycle backwards through the heights in the Phantom dataset to find the local maximum height. Do this by comparing the height of each data point to the height of the following data point (before it in the iteration). When the following data point is smaller than the current data point, it is the maximum. Find the maximum height and the time at which it occurs.

Find the Minimum Velocity Return to the perturbation onset. Assign initial minimum velocity as velocity at perturbation onset. Cycle through the derivative of the heights (i.e. velocity data) to find the minimum velocity (i.e. largest negative, or downward, velocity). Compare each velocity to current assigned minimum and if the current velocity is smaller, re-assign the minimum velocity to the current value. Get the minimum velocity, the time at which the minimum velocity occurs and the height at the minimum velocity. Some cases (e.g. P6) require cycling backwards through the data. This occurs if the perturbation onset is in a valley rather than near the peak.

Calculate the Perturbation Height Calculate the extrapolated height using symmetry about the minimum velocity,

$$
h_{E x t}=h_{v \min }+\Delta h
$$

and

$$
\Delta h=h_{v m i n}-h_{m i n}
$$


where

1. $\boldsymbol{h}_{\boldsymbol{E} \boldsymbol{x} \boldsymbol{t}}$ is the maximum height extrapolated using the symmetrical assumption

2. $\boldsymbol{h}_{\boldsymbol{v} \text { min }}$ is the height at the minimum velocity

3. $h_{\min }$ is the minimum height

Calculate the Symmetry Algorithm as difference between the maximum height and the extrapolated height.

Iterate through the Perturbations Iterate through the MIDI dataset to the next perturbation time and repeat.

\subsection{Perturbation Heights}

The algorithms were used to measure four different perturbation heights: basic max, basic onset, time shift and symmetry. The values for subjects 1 and 2 are shown in Tables 7.1 and 7.2 , respectively.

The results for the basic max and the time shift are always positive, as expected. However, both the basic onset and symmetry algorithms introduce negative values as the perturbation height. This implies that the downward perturbation raised the wrist height. A visual inspection of the graphs confirms that this is not the case, therefore it is concluded that the algorithms did not work in these cases. The negative values only occur for subject 1 .

Closer inspection of the perturbation heights reveals that there is no obvious pattern of change with increasing time (or bar number). This implies that there is no increase in impedance according to our measure. However, other mechanisms may have presented themselves that camouflage the increased impedance, such as movement in the upper limb that compensates for changes in impedance at the wrist. It must be noted that our measure of perturbation height at the wrist is not a measure of wrist joint stiffness. 
Table 7.1: Perturbation heights for subject 1

\begin{tabular}{|c|c|c|c|c|c|}
\hline Perturbation & Bar & Basic Max & Basic Onset & Time Shift & Symmetry \\
\hline P1 & 2 & 68.19 & 40.47 & - & 36.15 \\
\hline $\mathrm{P} 1$ & 23 & 93.25 & 51.77 & - & 33.81 \\
\hline $\mathrm{P} 1$ & 28 & 59.81 & 28.09 & - & 22.53 \\
\hline P1 & 49 & 79.14 & 39.56 & - & 32.03 \\
\hline $\mathrm{P} 2$ & 2 & 49.77 & 26.77 & - & 27.83 \\
\hline $\mathrm{P} 2$ & 23 & 74.81 & 50.02 & - & 30.58 \\
\hline $\mathrm{P} 2$ & 28 & 45.66 & 43.56 & - & 9.76 \\
\hline P2 & 49 & 64.85 & 35.76 & - & 27.93 \\
\hline P3 & 3 & 61.65 & 38.02 & - & 20.31 \\
\hline P3 & 24 & 72.65 & 52.44 & - & -11.50 \\
\hline P3 & 29 & 59.21 & 42.48 & - & 12.73 \\
\hline P3 & 50 & 73.21 & 50.88 & - & 5.21 \\
\hline $\mathrm{P} 4$ & 4 & 59.45 & 27.31 & - & 7.17 \\
\hline $\mathrm{P} 4$ & 25 & 65.55 & 39.76 & - & 39.75 \\
\hline $\mathrm{P} 4$ & 30 & 54.50 & 39.30 & - & -1.17 \\
\hline $\mathrm{P} 4$ & 51 & 47.45 & 30.23 & - & 33.50 \\
\hline P5 & 5 & 72.29 & 65.82 & 20.40 & -26.10 \\
\hline P5 & 6 & 58.04 & 54.19 & 2.86 & 16.65 \\
\hline $\mathrm{P} 5$ & 7 & 69.50 & 64.19 & 20.02 & 9.75 \\
\hline P5 & 8 & 76.28 & 72.48 & 30.23 & -2.66 \\
\hline P5 & 31 & 42.25 & 40.86 & 3.27 & 10.91 \\
\hline P5 & 32 & 63.32 & 57.14 & 11.00 & -14.46 \\
\hline P5 & 33 & 50.66 & 44.48 & 19.35 & -14.76 \\
\hline P5 & 34 & 61.70 & 50.36 & 20.55 & -1.45 \\
\hline P6 & 5 & 57.61 & -0.15 & - & -10.87 \\
\hline P6 & 6 & 53.66 & -0.35 & - & -7.62 \\
\hline P6 & 7 & 45.92 & 0.28 & - & 3.54 \\
\hline P6 & 8 & 54.93 & -1.03 & - & -8.59 \\
\hline P6 & 31 & 17.19 & 6.22 & - & 7.90 \\
\hline P6 & 32 & 7.50 & -6.39 & - & -6.64 \\
\hline P6 & 33 & 14.94 & 5.69 & - & -9.09 \\
\hline $\mathrm{P} 6$ & 34 & 38.09 & 2.88 & - & -18.99 \\
\hline
\end{tabular}


Table 7.2: Perturbation heights for subject 2

\begin{tabular}{|c|c|c|c|c|}
\hline Perturbation & Bar & Basic Max & Basic Onset & Time Shift \\
\hline $\mathrm{P} 1$ & 2 & 31.47 & 16.22 & - \\
\hline $\mathrm{P} 1$ & 23 & 26.04 & 14.62 & - \\
\hline $\mathrm{P} 1$ & 28 & 11.49 & 10.73 & - \\
\hline $\mathrm{P} 1$ & 49 & & & - \\
\hline $\mathrm{P} 2$ & 2 & 13.82 & 10.81 & - \\
\hline $\mathrm{P} 2$ & 23 & 15.91 & 12.37 & - \\
\hline $\mathrm{P} 2$ & 28 & 23.02 & 22.89 & - \\
\hline $\mathrm{P} 2$ & 49 & & & - \\
\hline P3 & 3 & 17.51 & 11.42 & - \\
\hline P3 & 24 & 20.97 & 14.14 & - \\
\hline P3 & 29 & 29.68 & 19.71 & - \\
\hline P3 & 50 & & & - \\
\hline $\mathrm{P} 4$ & 4 & 15.74 & 12.62 & - \\
\hline $\mathrm{P} 4$ & 25 & 18.53 & 15.28 & - \\
\hline $\mathrm{P} 4$ & 30 & 25.57 & 23.91 & - \\
\hline $\mathrm{P} 4$ & 51 & & & - \\
\hline P5 & 5 & 25.03 & 19.27 & 17.66 \\
\hline $\mathrm{P} 5$ & 6 & 27.20 & 22.94 & 22.58 \\
\hline P5 & 7 & 23.11 & 15.06 & 14.67 \\
\hline P5 & 8 & 28.08 & 24.33 & 20.72 \\
\hline P5 & 31 & 32.20 & 24.21 & 12.30 \\
\hline P5 & 32 & 34.07 & 26.67 & 17.91 \\
\hline $\mathrm{P} 5$ & 33 & 32.21 & 25.65 & 17.27 \\
\hline $\mathrm{P} 5$ & 34 & 34.97 & 25.59 & 13.83 \\
\hline $\mathrm{P} 6$ & 5 & 11.79 & 10.94 & - \\
\hline $\mathrm{P} 6$ & 6 & 13.20 & 6.70 & - \\
\hline $\mathrm{P} 6$ & 7 & 9.82 & 6.91 & - \\
\hline $\mathrm{P} 6$ & 8 & 17.65 & 10.19 & - \\
\hline P6 & 31 & 8.13 & 6.76 & - \\
\hline $\mathrm{P} 6$ & 32 & 6.26 & 4.66 & - \\
\hline P6 & 33 & 9.16 & 4.75 & - \\
\hline $\mathrm{P} 6$ & 34 & 1.39 & 0.81 & - \\
\hline
\end{tabular}


The basic algorithm has two results; basic max and basic onset. Basic max is the simplest of the algorithms, as it calculates the difference between the local maximum peak height and the local minimum valley height. All values resulting from the basic max algorithm can be compared directly to each other to determine if there has been a change in displacement. However, they do not give a true indication of the displacement resulting from the perturbation as they include any motion that occurred due to the natural undulations occurring for the unperturbed notes.

Basic onset gives a truer representation of the reaction to the perturbation as it only calculates the height difference between the perturbation onset and the local minimum. Since perturbations can occur at any point along the period of the wave these values cannot be directly compared to each other. Figures 7.5 and 7.6 demonstrate the impact of the location of perturbation onset. Figure 7.5 shows a case (subject 1 , reading 1 , perturbation 1) where the perturbation onset occurs partway along the downward slope of the wave. This resulted in a positive perturbation height. However, Figure 7.6 shows a case (subject 1 , reading 1 , perturbation 6 , bar 8 ) where the perturbation onset occurs after the valley of the wave. This case resulted in a negative perturbation height because the algorithm searches for a local minimum following the perturbation onset. In this case, it finds a minimum which is higher than the height at the perturbation onset.

The time shift algorithm compares the differences between maximum peak height and minimum valley height for perturbed and unperturbed cases of the same passage. There was just one opportunity to use this algorithm in the supplied piece of music, at perturbation 5 . Every result calculated with this algorithm gave a positive perturbation height, indicating that the algorithm functioned well in all cases. Additionally, this algorithm gives the truest representation of the displacement due to perturbation, since it eliminates vertical motion due to the natural undulations during playing.

For this algorithm, data was normalized along the same time axis to compare the perturbed and unperturbed cases of the same passage. Examples of aligning the data by 


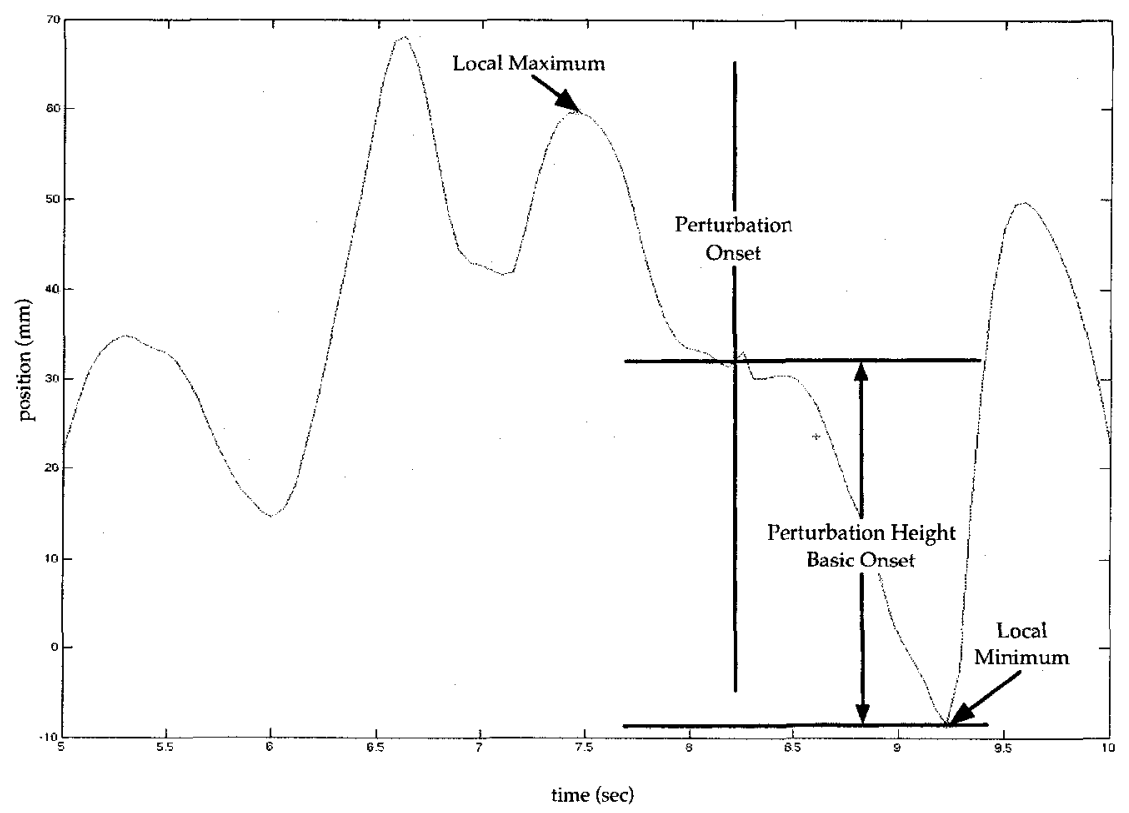

Figure 7.5: Example of the basic onset algorithm functioning well. Figure shows the perturbation height for basic onset for subject 1, reading 1 , perturbation $1 ; 40.47 \mathrm{~mm}$. In this case the perturbation onset occurs partway down the wave.

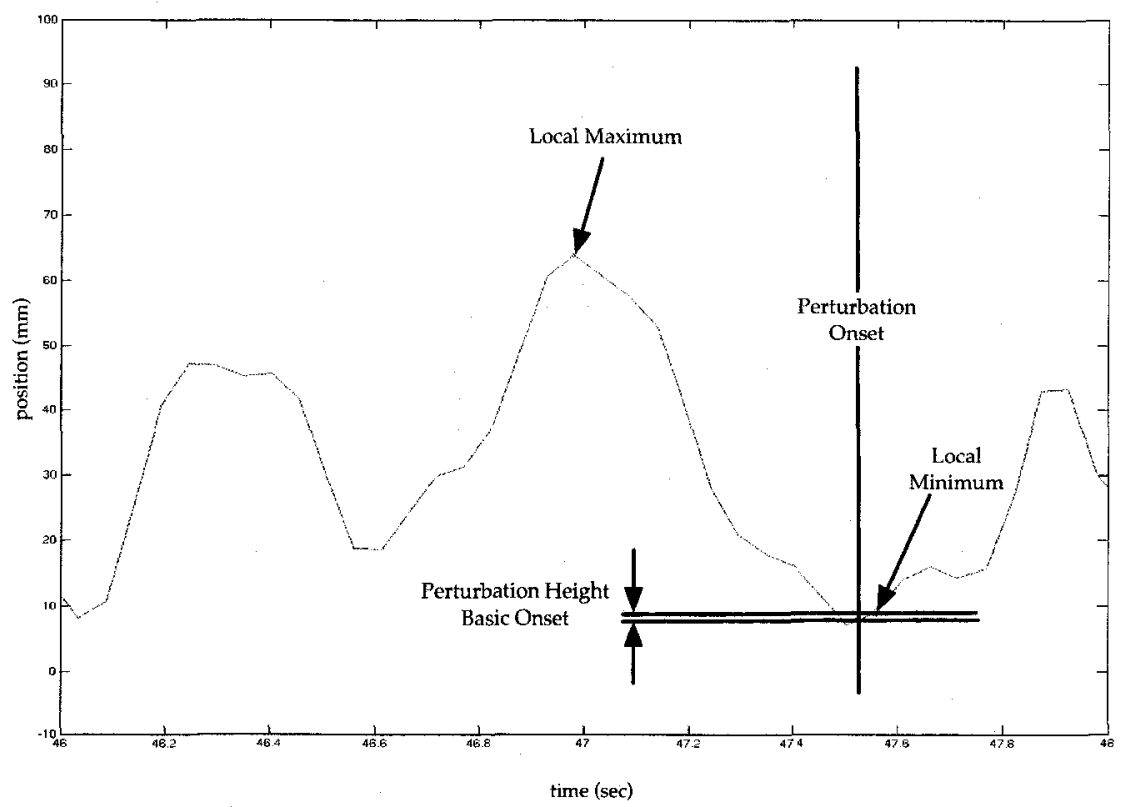

Figure 7.6: Example of the basic onset algorithm not functioning. Figure shows the perturbation height for basic onset for subject 1 , reading 1 , perturbation 6 , bar $8 ;-1.03$ $\mathrm{mm}$. In this case the perturbation onset occurs after the valley of the wave. 
normalizing the times are shown in Figures 7.7 and 7.8. Data was normalized by aligning the two peaks for each case, or adjusting the period of the wave.

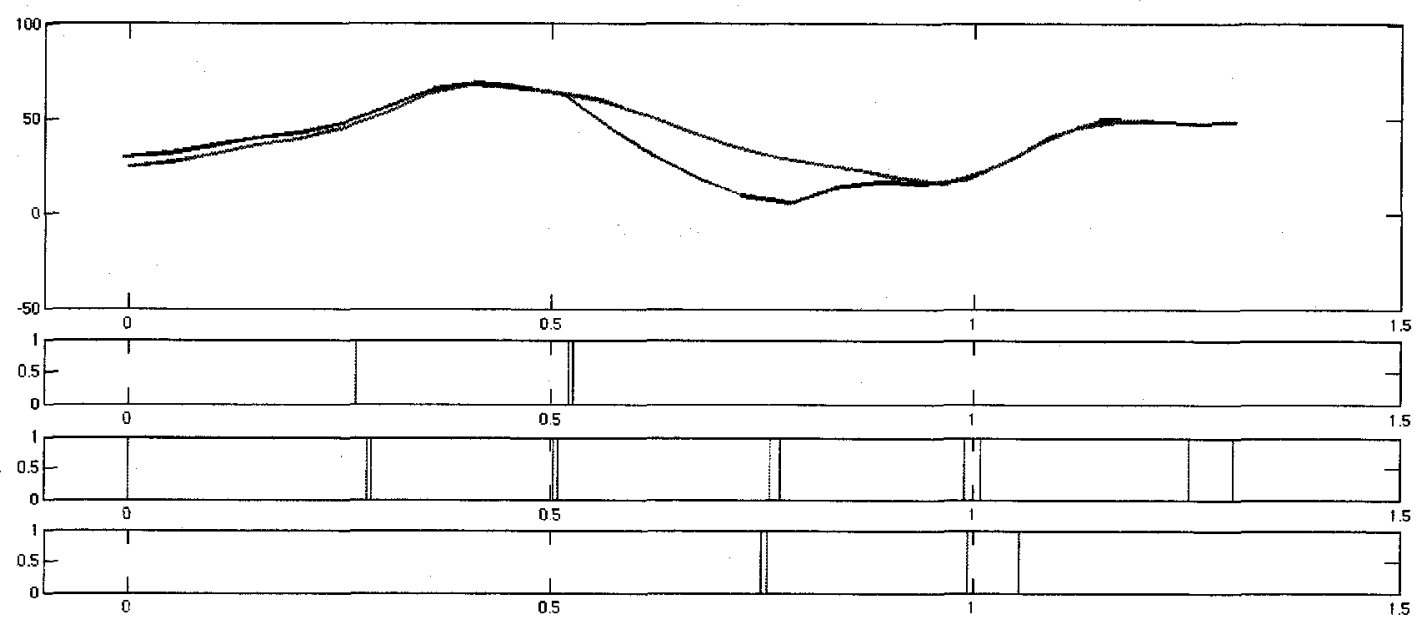

Figure 7.7: Example of data normalization for subject 1; this case is reading 2, bar 6 . The difference between the two max-min heights is $11.00 \mathrm{~mm}$.

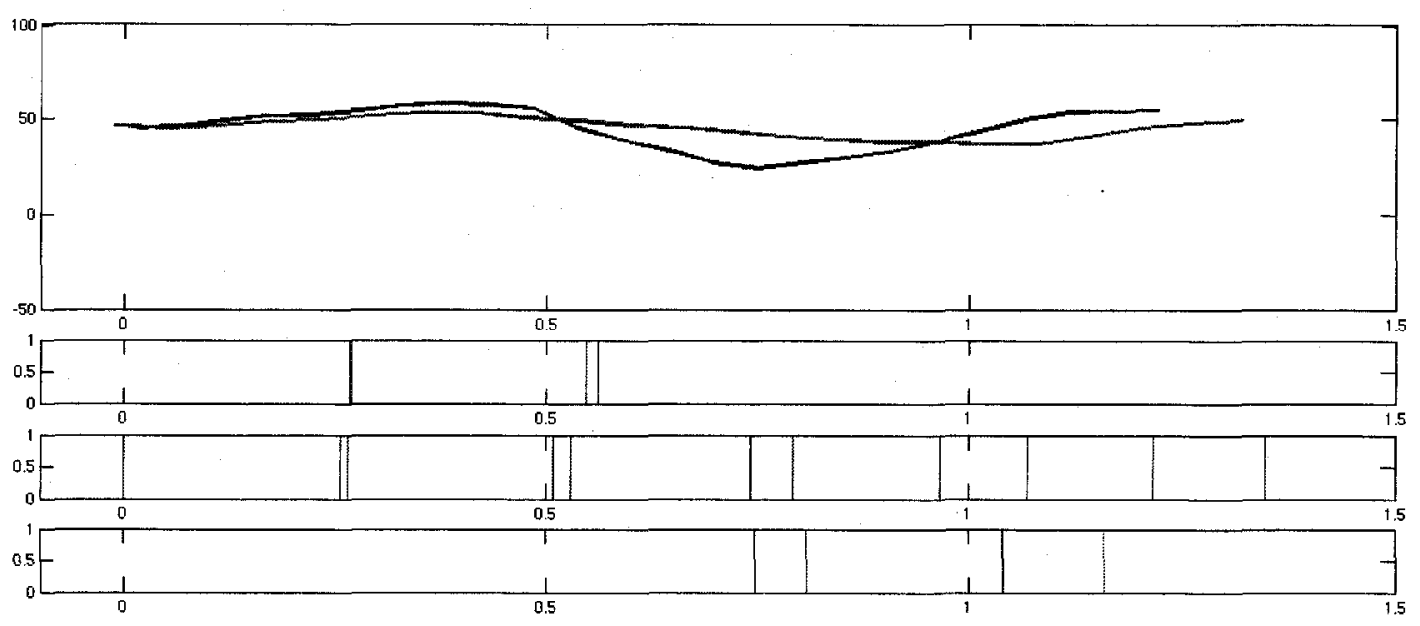

Figure 7.8: Example of data normalization for subject 2 ; this case is reading 2, bar 6 . The difference between the two max-min heights is $17.91 \mathrm{~mm}$.

In this case the assumption is made that every read through the data would have an identical response if left unperturbed. The ideal situation would be to have many sets of unperturbed data for an identical passage which could be averaged before comparison with the perturbed data. 
The symmetry algorithm is based on the assumption that the waves are symmetrical. Each half-wave is assumed to be symmetrical about the point of the largest slope. The algorithm is only interested in decreasing slope so it looks for the largest negative velocity, or the minimum velocity. Figure 7.9 demonstrates the theory in a working example (subject 1 , reading 1 , perturbation 1 , bar 2). In this case the perturbation onset occurs partway along the decreasing slope of the wave. The algorithms finds the local minimum and the point of minimum velocity and works backwards up the slope to extrapolate the maximum height that should have occurred without the perturbation. The extrapolated height is below the actual height and so the displacement due to perturbation is calculated as the difference between the actual maximum and the extrapolated height. In this case the perturbation height is positive: $36.15 \mathrm{~mm}$.

Another example, shown in Figure 7.10, demonstrates that the assumption that the waves are symmetrical about the largest negative velocity (largest negative slope) must be false. This case (subject 1 , reading 1 , perturbation 5 , bar 5 ) has a perturbation onset that occurs near the peak of the wave. The largest negative velocity is much closer to the peak than to the valley and therefore the extrapolated height is well above the actual measured height, resulting in a negative perturbation height: $-26.10 \mathrm{~mm}$. Since the downward perturbation did not cause the wrist to lift, it must be concluded that the assumption of symmetry is false. 


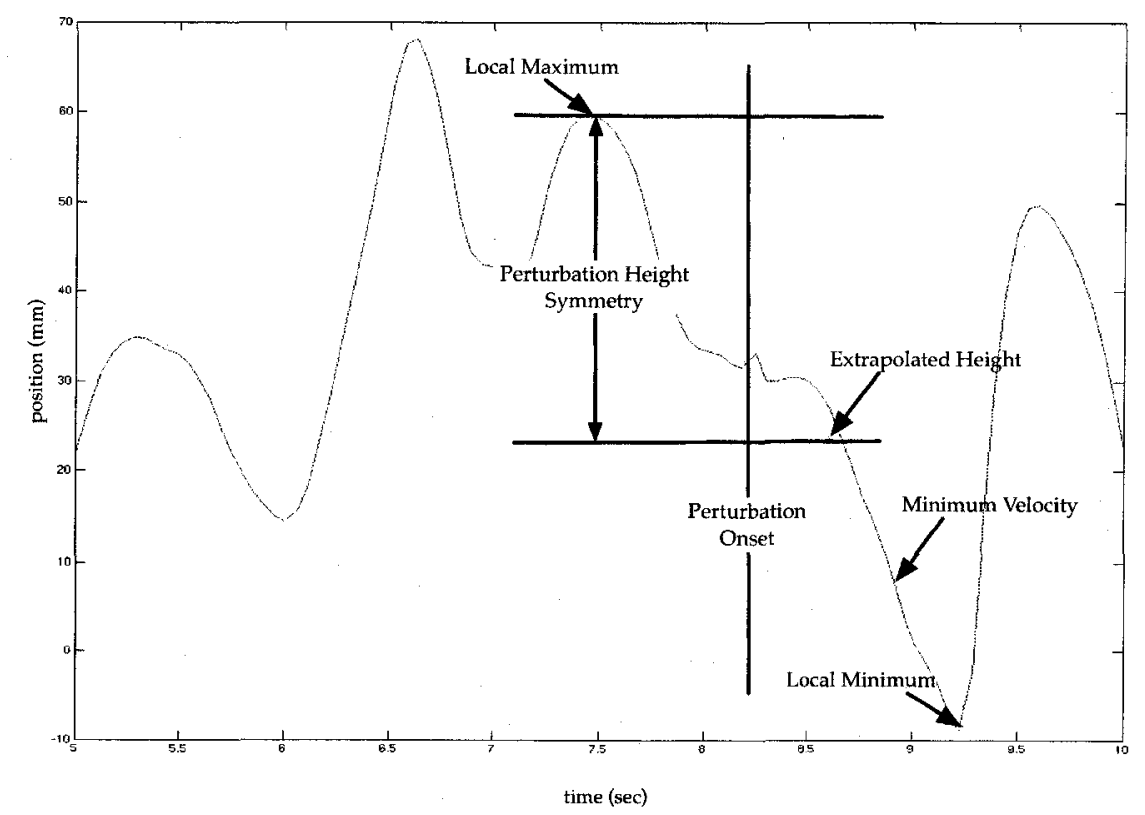

Figure 7.9: Example of the symmetry algorithm functioning well. Figure shows the perturbation height for symmetry for subject 1, reading 1, perturbation 1: $36.15 \mathrm{~mm}$. In this case the perturbation onset occurs partway down the wave.

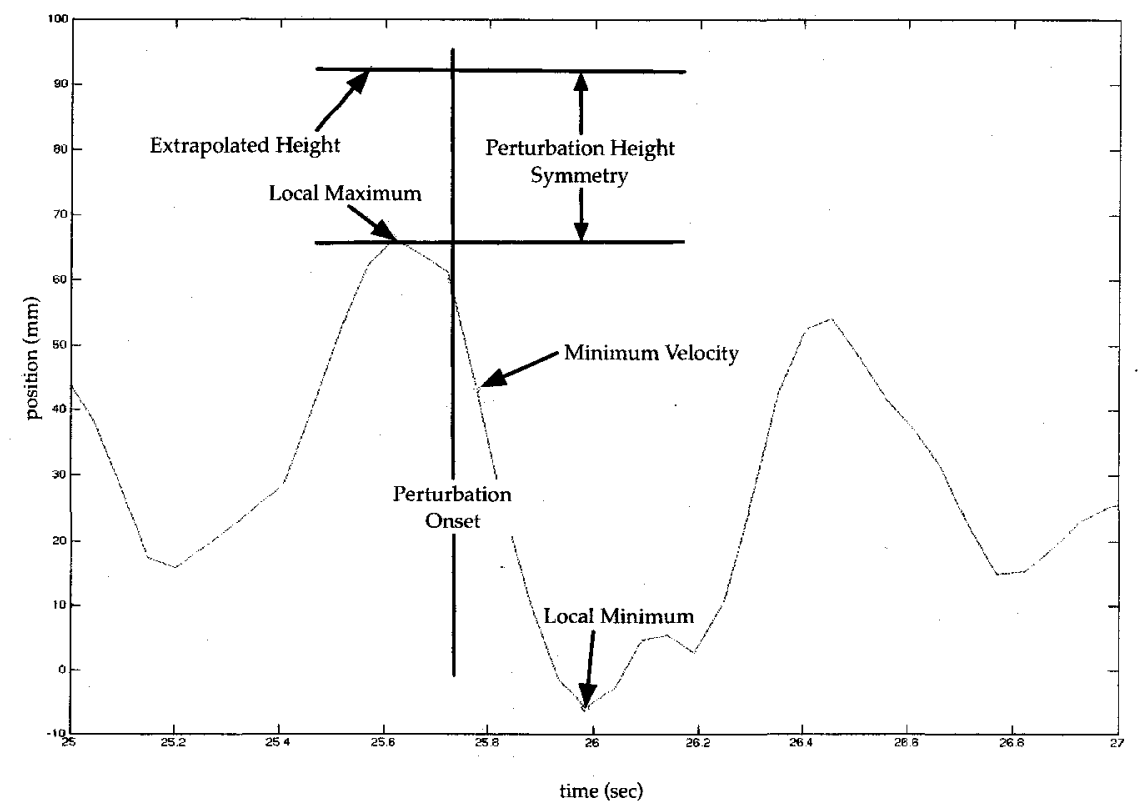

Figure 7.10: Example of the symmetry algorithm not functioning. Figure shows the perturbation height for symmetry for subject 1, reading 1, perturbation 5, bar 5: -26.10 $\mathrm{mm}$. In this case the perturbation onset occurs near the peak of the wave. 


\section{Chapter 8}

\section{Discussion}

This is a novel study that assesses a preliminary approach to determining impedance at a joint during the complex task of piano playing. Current estimations of impedance are made in either a quasi-static or static state, or during a simple movement such as finger tapping or targetted movements in a controlled environment.

The piano community uses the term stiffness in a general way to denote impedance, which includes not only a stiffness term, but also a damping term and an inertia term. The

force is defined in Equation 8.1, where $\boldsymbol{F}$ is the force, $\boldsymbol{k}$ is the stiffness coefficient, $\boldsymbol{x}$ is the displacement, $\boldsymbol{b}$ is the damping coefficient, $\boldsymbol{v}$ is the velocity, $\boldsymbol{m}$ is the mass, or inertial component, and $\boldsymbol{a}$ is the acceleration. Static conditions assume a negligible contribution from the damping and inertial elements, but because piano playing is a dynamic system, the damping and inertia cannot be ignored.

$$
F=k x+b v+m a
$$

The success of the impedance evaluation is dependent on the perturbation applied. Impedance is measured by applying a known force and measuring the reaction at that point. In this study, the perturbation was applied to the wrist with a downward direction, a $3 \mathrm{~N}$ magnitude and a duration determined by the length of the key depression.

As a selected note in the piece was depressed, the Phantom was triggered to apply the perturbation. As the note was released it was triggered again, but this time to stop applying 
the perturbation force. Therefore at note onset the wrist position of a perturbed note should be similar to any note in the piece, but at key release the wrist position is expected to be lower due to the displacement reaction to the applied force. Statistical measures of the position of the wrist at key release for perturbed and unperturbed notes showed a significant difference with $95 \%$ confidence for each of the two subjects. The statistical analysis of the data was conducted under the assumption of stationary statistical properties. Since the reaction was significantly different from the normal motions of playing unperturbed notes, the amplitude is deemed appropriate for this study.

The duration of the perturbation was linked to the duration of the key depression. The original constraints for the piece specified that a quarter note should last no longer than $500 \mathrm{~ms}$. Both subjects chose a similar tempo for their trials but it was slightly slower than the tempo set for the piece. As well perturbations were not only applied to quarter notes, but also to half notes. The duration of the notes and therefore of the perturbations lasted over $1 \mathrm{sec}$ in some cases. The inclusion of a metronome during playing is recommended to eliminate errors in tempo selection during future trials.

Signal transmission along a nerve is not instantaneous. It is known that a nerve signal takes about $400 \mathrm{~ms}$ to travel from the peripheral nerves to the brain, be processed and travel back again. This is the amount of time that it takes a voluntary response to a disturbance to occur in the arm. The stretch reflex ensures that muscle length and tension are maintained and is responsible for lengthening a muscle in response to an external disturbance, which is the displacement that is measured in this study. The stretch reflex is the simplest reflex and it only requires the nerve signal to travel as far the spindle fibres contained in the muscle, so it occurs much faster than voluntary responses.

Impedance measurements normally limit the duration of the perturbation to $400 \mathrm{~ms}$ or less so that the measurement includes only reflex activity and not any voluntary response. Although the duration of the perturbation lasted much longer than $400 \mathrm{~ms}$ in some cases, there was no evidence of a voluntary response to the perturbations so the duration of the 
perturbation is deemed appropriate for this study.

The Phantom Omni is a haptic device that applied the known force to the wrist. The force application was triggered by the depression of particular trigger notes in the supplied music. Throughout the experiment the Phantom recorded the position of the wrist in three dimensions with a coordinate system linked to its orientation. The Phantom also recorded forces throughout the experiment, but these appear to be a log of either 'on' or 'off' for the specified force, as opposed to an instantaneous measurement of the force. Since the force was input to the device, this was the value it recorded. Although the force was consistent throughout all of the trials, the logged magnitude may not be accurate and was not be used for impedance calculations.

The subjects did not find the apparatus overly bulky when it was attached to their forearm. The apparatus and perturbations did not inhibit their playing.

The Polaris was used to collect position data of the finger and wrist as well as the piano key and the Phantom's task arm. The Phantom was measured to allow synchronization of the data between the Phantom and the Polaris data. It is evident when comparing the position data of the Phantom (Figures 6.1 and 6.2) to the position data of the Polaris (Figures 6.5 and 6.6) that the Polaris had an insufficient sampling frequency to provide useful data. The Polaris data was not analyzed any further.

The displacement reaction occurred as a result of the applied perturbation; changes in displacement must be due to changes in the impedance at the wrist. The impedance is inversely proportional to the measured displacement; any increase in displacement indicates a decrease in impedance and any decrease in displacement indicates an increase in impedance. This measure is indicative of the driving point impedance, it is not a only a stiffness because of the dynamic aspects of piano playing and it is not a joint stiffness because it includes the effects from all the other joints in the upper extremity. It gives a measure of the external stiffness effects, as well as damping and inertial effects. 
The data was only analyzed in the vertical y-direction, but this is justified by the limited amount of movement in the $\mathrm{x}$ and $\mathrm{z}$ directions of the horizontal plane, as shown in Figures 6.3 and 6.4. The position data is oriented with respect to the Phantom Omni. The y direction is vertical, the $\mathrm{x}$ direction runs laterally across the Phantom and the $\mathrm{z}$ direction runs from front to back in the median plane of the Phantom.

Visual observation of the results from subject 1 reveal that the playing style changed with tempo. At the slow tempo during the first two bars of half notes, one oscillation period encompassed only one note. As the tempo increased while playing quarter notes during bars three and four, the oscillations changed so that one oscillation period encompassed two notes. Similarly with the next tempo increase to playing triplets in bars five through eight, one oscillation period spanned three notes. The changes in note groupings reflect the time signature of the piece, which is measured in half notes. The groupings appear to represent the stressed beats or downbeats.

Four different measures of perturbation height were calculated: 'basic max', 'basic onset', 'time shift' and 'symmetry'. The basic max and time shift algorithms always produced a positive result, while the basic onset and symmetry algorithms produced some negative values for subject 1 .

The measure called 'basic max' calculated the difference between the maximum and minimum heights of the note. The unperturbed playing of the subjects included vertical motion even during unperturbed playing. The algorithm therefore included the displacement due to the natural unperturbed motions of playing as well as the displacement due to the perturbations. The two subjects differed in the playing style as recorded in the vertical displacement of the wrist. The wrist movement of subject 1 includes prominent oscillations while that of subject 2 is fairly flat by comparision. Due to the inclusion of both unperturbed and perturbed playing in this measurement method, it cannot be used for comparison between the subjects. 
The measure called 'basic onset' was similar to basic max but calculated the difference between the wrist height at perturbation onset and the minimum height for that note. This measure works well for comparing the same note in a passage, but is not transferable to other notes. The reason is evident when looking at the oscillations of subject 1 ; wrist height varied depending on which note in the grouping was played. It is not expected to find the same wrist height at note onset for, for example, the first and last notes in the grouping. This measure is valid only for comparing various trials of the same occurrence of a particular note.

The 'time shift' measurement compared two identical passages that were repeated in the music. A note in the passage should be perturbed in one case and unperturbed in the other case to allow comparison of the perturbed displacement with the unperturbed displacement. The passages should be near one another in time so that the impedance can be assumed to be constant. There was only one instance in the piece where an identical passage was played sequentially in the perturbed and unperturbed cases. An ideal case would be to have a trial where the piece is played in its entirety without perturbations so that every perturbation could be compared to an identical unperturbed case.

The normalization of the 'time shift' data only adjusted the data in the horizontal direction. The graphs in Figures 7.7 and 7.8 are examples of the normalized data and it is evident from visual inspection that the vertical position of the wrist appears repeatable, other than the displacement due to the perturbation.

The 'symmetry' measurement is based on the assumption of symmetry in the oscillatory motion of playing. The point of symmetry was assumed to be at the point of the largest negative velocity; the slope of interest was negative and so the velocity of interest was also negative. Since the results from this measurement are almost half negative values, indicating a rise in wrist height, the assumptions of the algorithm are accepted to be incorrect.

In summary, 
- the basic algorithms do not produce results that are transferable between subjects,

- the symmetry algorithm cannot be used in all conditions since the algorithm assume an oscillatory motion of the wrist, besides which the algorithm is based on incorrect assumptions, and

- the time shift algorithm can be used for any playing style and isolates the effects of the perturbation from the normal deviations in motion of the wrist.

The time shift algorithm is therefore recommended for use in future studies.

Inspection of the results in Tables 7.1 and 7.2 reveals no obvious trends in the data of both subjects. The expected result was to have decreasing displacement, or increasing impedance, with time. The music incorporated increased dynamic levels and increased tempo into the increased duration, which were all hypothesized to create increased impedance.

The data of subject 1 suggests the opposite effect occurs throughout the piece, a weak trend of increasing displacement with increased duration in each trial individually, indicating a decrease in impedance during each trial. The subject may have been nervous at the beginning of the trial and therefore exhibited increased impedance which decayed throughout the trial; the subject had never undergone a perturbation prior to the first trial. Another possible explanation for this increased displacement is that the subject learned the style of the piece and was able to adapt to it, invoking the decreasing impedance effects of learning. No trend of increasing (or decreasing) displacement was found for subject 2.

When comparing the two trials of subject 1 , it is evident that the second trial had smaller displacements in reaction to the perturbation, as demonstrated in Figure 8.1 using the results from the 'basic max' algorithm. This implies an increase in impedance from one trial to the next. There was no evident increase in impedance during the first trial so it is unexpected to see an increase from one trial to the next. Subject 2 demonstrated no 
evident change in impedance between trials. For both subjects, the trials are comparable in terms of which notes have the highest and lowest displacements.

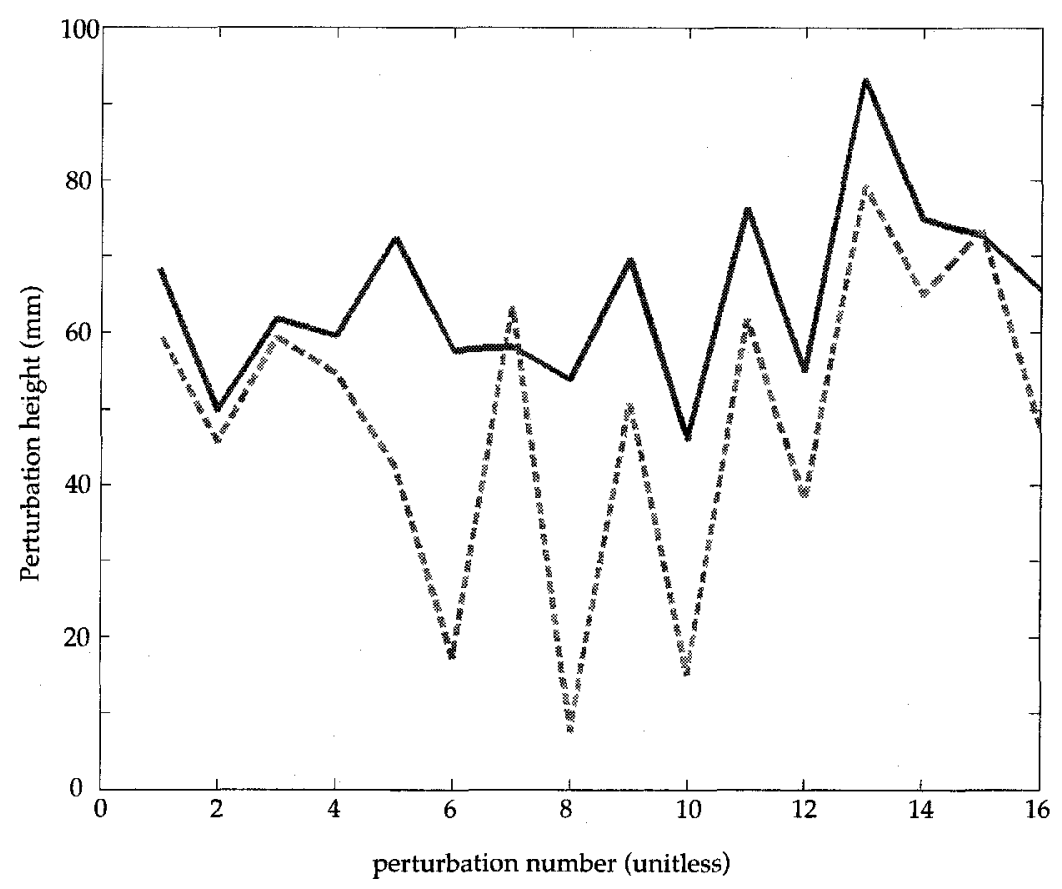

Figure 8.1: Comparison of perturbation heights for subject 1. The solid line represents the first trial and the dashed line represents the second trial.

Between trials it is also possible to look for trends due to learning. The repetition of an act makes motor planning easier and the result is expected to be smoother and to expend less energy through impedance. The hypothesized increase in displacement is not found between trials in either subject; the data from subject 1 suggests an opposite trend.

It should be noted that these are simply trends found in the data, not correlations between parameters. The results of the study cannot be generalized due to the small sample population.

The two subjects in this study had very different playing styles, as is evident in the graphs of the vertical position of the wrist. Since subject 1 had a much larger oscillatory 
amplitude, the perturbation heights are difficult to compare. The basic algorithm cannot be used for comparison because it includes height due to the natural motions of the wrist. The symmetry algorithm cannot be used because it was not calculated for subject 2 . The only values for comparison are the 8 perturbation heights measured using the time shift algorithm.

The average and standard deviation for the time shift results of subject 1 are $15.96 \mathrm{~mm}$ and $9.48 \mathrm{~mm}$, respectively and those for subject 2 are $17.12 \mathrm{~mm}$ and $3.46 \mathrm{~mm}$. The subjects therefore presented similar levels of impedance, although subject 1 had more variability. In general, subject 1 had greater perturbation heights, and therefore smaller sitffness levels, but also presented two outlying low perturbation heights representing high impedance levels.

The music composed for this study was unable to induce the hypothesized changes in impedance in these subjects. A possible explanation is that the parameters were insufficient to induce increased impedance, both subjects commented that they felt no increase in impedance during playing. Another possibility is that impedance was created during the faster passages in bars 17-20. Both subjects commented that these bars were difficult due to the lack of pattern that was present in the majority of the piece. Analysis was not performed on the faster measures because their duration was too short to record the reflex response to the perturbations. The subjects repeated bars $1-4$ at the end of the trial and were perturbed as they were at the beginning of the piece. The impedance may have dissipated at the return to the slower tempo due to an alleviation of the cause - the increased tempo and difficulty of bars 17-20.

The parameters included in the piece were increased duration, increased loudness and increased tempo, all of which are hypothesized to increase impedance. Exaggerated versions of these parameters may be required to induce impedance within the short 1-minute timeframe allotted per trial, or the trial length could be extended to increase the duration of playing. Other hypothesized methods of inducing impedance could be included, such as abduction of the fingers, or finger independence during key strike, as during Hannon or 
Czerny exercises.

\subsection{Outline of the Proposed Experimental Methodology}

The study evolved into a set of preliminary experiments due to the large number of changes recommended to be made to the methodology. The most important changes are to alter the piece in a manner that will induce a impedance effect, to measure the position of the limb over time at each joint and to have accurate force measurements required for impedance calculations. A detailed description of the proposed methodology follows.

The subjects will be university-level piano majors. Population data will be collected from subjects using a questionnaire; the data will include:

1. Age;

2. Gender;

3. Number of years playing piano / age of first instruction;

4. Dominant hand (all tasks will be performed using the right hand);

5. Duration of daily practice / the number of days per week of practice;

6. Current sample practice repertoire;

7. Injury history; and

8. Formal qualifications including program major and any degrees earned, to differentiate among the student population.

The subject will enter the piano lab without any warm-up exercises having been completed. They will be fitted with retro-reflective markers along their right upper limb, from the shoulder to the finger tip, the specific locations are outlined in Table 8.1. Markers will also be placed on the piano key and the force application device. 
Table 8.1: Marker Definitions for the Proposed Methodology

\begin{tabular}{|c|c|}
\hline Number & Definition \\
\hline 1 & Piano key played by the right index finger \\
\hline 2 & Stylus of the Phantom \\
\hline 3 & Cuticle of the nail of the right index finger \\
\hline 4 & $\begin{array}{l}\text { Distal interphalangeal (DIP) joint of the right index finger, at the head of the } \\
\text { middle phalanx }\end{array}$ \\
\hline 5 & $\begin{array}{l}\text { Proximal interphalangeal (PIP) joint of the right index finger, at the head of } \\
\text { the proximal phalanx }\end{array}$ \\
\hline 6 & $\begin{array}{l}\text { Metacarpophalangeal (MCP) joint of the right index finger, at the head of the } \\
\text { metacarpal bone }\end{array}$ \\
\hline 7 & Styloid process of the ulna of the right wrist \\
\hline 8 & Lateral epicondyle of the humerus at the right elbow \\
\hline 9 & Acromion of the right shoulder \\
\hline
\end{tabular}

The marker on the force application device will be used to synchronize data from the motion capture device and the force application device. The markers on the knuckles and the fingertip can be used to determine the number of effective links in the mathematical model of the arm. This is accomplished by analysing the motion capture data to determine which joints are bending during playing.

At this time the subject is ready to commence playing. At the beginning of each trial or warm-up exercise, the subject will lift their right arm before playing. This will allow the data from all devices to be synchronized. They will complete supplied warm-up exercises, such as simple 5-note repeating passages. Once the warm-up is complete the subject will sight-read a supplied piece of music; the tempo will be set to 120 beats per minute to a quarter note, using a metronome that will operate throughout the piece. No perturbations will be applied to the subject during this first trial, which will act as a control trial for the subject. The subject will either play the piece in its entirety or will indicate a need to stop if they feel "stiff". The performance will be documented on video, recording the hand in the sagittal plane, and using a motion capture device with a high frequency of data collection. 
After a period of rest the subject will undergo another trial of playing the same piece. In this trial, perturbations will be applied to the subject's right wrist when selected trigger notes are played. The subject will not be made aware of which notes will be triggered. Selection of trigger notes will be done in a way that maximizes the ability to look for trends in data. For example, in the supplied piece, the passage 'A-G-F-G' occurs many times. In the first four bars it occurs 3 times and so it is recommended to trigger the first $\mathrm{G}$ of the passage during each repetition, that is it is recommended to perturb notes 4,12 and 24 . Each perturbation can then be analyzed with respect to itself, using the time shift algorithm and the data from the first trial, as well as with respect to the other perturbed occurrences of the passage throughout the piece. That is, a perturbation height can be calculated for notes 4, 12 and 24 by comparing them to notes 4,12 and 24 of the unperturbed trial. Then, the perturbation heights can be compared to each other to determine if there was any increase in impedance with the duration of playing or the tempo increase.

Data will be analyzed in the vertical direction using the time shift algorithm for the calculation of perturbation heights. 


\section{Chapter 9}

\section{Conclusions and Recommendations}

\subsection{Conclusions}

The main conclusions of the study relate to the goals outlined in Chapter 1 . The first goal was to develop a means of measuring changes in impedance during piano playing. The corollary to this was to obtain a piece of music that would generate an increase in impedance during playing.

A vital aspect of impedance measurements is the application of an appropriate perturbation, in both magnitude and duration. Two pieces of evidence lead to the conclusion that the perturbation magnitude is appropriate for creating measurable displacement reactions: (1) the wrist position at the end of the perturbation was significantly different from the wrist position at the end of an unperturbed note and (2) the reactions to the perturbation were much larger than the displacement due to the compression of soft tissues in the arm. The criteria for an acceptable perturbation duration is that it is short enough to eliminate the effects of voluntary reactions. Because there was no evidence of voluntary responses to any of the perturbations it can be concluded that the perturbation duration was appropriate.

The wrist height fluctuated during the performance, so obtaining a measure of displacement was not straight-forward. Four different measures of the displacements were calculated. The measure called 'time shift' was deemed to be the most accurate and reliable measurement of displacement because it compares perturbed and unperturbed notes that appear at the same point in a repeated passage. Careful selection of notes to perturb is 
required to reap the full benefits of this measure. The ideal would be to have subjects play the piece through in its entirety to have the ability to compare the displacements for every perturbed note.

Finally, the piece did not cause a significant increase in impedance for these subjects, measurable or otherwise. The subjects commented that there was no feeling of increased impedance while performing the task and the data shows no significant increase in the impedance measure. Although there is evidence of changes in impedance there is no correlation between changes in impedance and any effects invoked by the music, such as duration, tempo, or loudness (dynamic level).

The study is considered successful because it achieved the goal of developing a methodology that would measure changes in impedance during piano playing, a measurement that has never been taken before.

\subsection{Recommendations}

While researching the best place to delve into biomechanical piano research, numerous projects were suggested. These other projects can offer further insight into impedance, stiffness and relaxation from a pianist's point of view.

\section{Continuation of Preliminary Work}

Various improvements to the experimental methodology are outlined in Chapter 8. Among them are to: (1) alter the music so that it induces an increase in impedance, (2) measure the position of all joints in the upper extremity, (3) use a device that outputs an accurate reading of the applied force, and (4) capture the performances on video.

With sufficient data, correlations could potentially be found between impedance and parameters such as posture, technique, years of experience, or instrument type - the study could be expanded to playing on the lighter actions of the clavichord or harpsichord. 
Impedance is also thought to correlate inversely with learning, subjects could undergo numerous trials to look for trends due to learning. After practice, the subjects may also be able to play at a faster tempo, which may enable an increase in impedance. Younger subjects may offer insight into learning as well, since the music would be more challenging for them. A range of ages and skill levels for the subjects could give a broad spectrum of learning throughout all levels of piano playing. Players also have a dominant hand, although even if the left hand is initially dominant, it may eventually become the non-dominant hand due to the more demanding role of the right hand. Measurements taken of the left side may produce higher levels of impedance than measurements of the right side.

\section{Instrumented Piano Key}

The measurement of force at the endpoint could be accomplished by instrumenting a piano key with force transducers. This would allow accurate force and velocity measurements to be taken without using a MIDI system. The force sensors can gather accurate data from either on top or below the key since the key is assumed to transfer the entire load to the keybed.

Placement of the sensor between the keybed and the key bottom has been attempted using a dynamic pressure measurement system, such as F-scan [106]. In this study the felts were removed from under the keys to create space for the force-sensing equipment and the forces were measured under the entire range of keys that were played, as opposed to from under a single key. This set-up could be improved to maintain the natural feel of the felts beneath the keys by using a smaller sensor with a one-to-one key-to-sensor ratio.

Placement of the sensor on top of the key could allow for the measurement of directional forces. Horizontal force components were measured during tapping of a keyswitch [72] and it is possible that key depression contains a similar horizontal component. So far piano biomechanics has assumed a purely vertical force application to the piano key. 


\section{Extension of Existing Theoretical Models}

Theoretical models of the hand are currently used to estimate stiffness and strain in joints and in tendons. These models have simplifying assumptions that can be refined to include recent neuromuscular control findings. Some of the limitations of the current models are: they only calculate conditions during keystrike for the flexor muscles but not during key release for the extensor muscles; they do not account for muscular adaptation that undoubtedly occurs during piano playing; they assume quasi-static motion due to small inertial effects; and they are limited to two dimensional motion in the sagittal plane that does not allow for three-dimensional loading using abduction and adduction.

There are two types of muscles used in finger control: intrinsic muscles and extrinsic muscles. Intrinsic muscles are located within the hand itself and control actions such as finger abduction and adduction. The extrinsic muscles are located in the arm and only their tendons cross the wrist joint into the hand. These muscles control finger flexion and extension, and are therefore integral in piano playing, as well as numerous other tasks. The existing theoretical models should be extended to include these muscles to give an accurate picture of the physiology of finger movements. That is, the models that currently only include the finger joints should be expanded so that they cross the wrist joint.

Data from a combination of the first two projects would include joint positions and forces at two points in the limb. Having force data at two points will reduce the redundancy of the model and be ideal for input into a mathematical model that is expanded across the wrist joint.

\section{Technical Solutions to Pain}

Many studies suggest to reduce the forces in the tendons and muscles. So far the focus of this work has been to alter the posture of the finger into a position that minimizes these forces. Technical solutions have not been explored as a solution to this force reduction. When the modern piano was invented in the 19th century it was advantageous because it 
had bigger sound, but this was at the cost of increasing string tension and incorporating a heavier action, both of which demand an increased applied force for key depression.

Other than reducing forces by changing the action, the physical arrangement of the keyboard could be improved. Ergonomics has improved the layout of the computer keyboard for typists, who are known to also suffer from overuse injuries. The current layout of the piano keyboard introduces biomechanical problems requiring extension and abduction of the fingers to reach the keys.

\section{Measurement of Shoulder EMG during Piano Playing}

EMG can be used as a measure of stiffness or a measure of energy expenditure. Since muscles are active components of the body, they consume energy when they are tense. Taking measurements of the electrical activity in the shoulder muscles during playing can give a scientific basis for a particular seated posture. Measurement of the trapezius and levator scapulae, the muscles that lift or shrug the shoulders, could be particularly interesting since many piano students are identified as being tense based on their shoulder height.

\section{Effects of Seating Position}

Many pedagogues suggest that it is important to support the feet during playing. Measuring the forces of the thighs on the bench and of the feet on the floor could determine the effect of supporting the feet of younger, or shorter, students. Hypotheses could be made as to how the posture of the child is altered by supporting the feet. Unsupported feet add weight to the thighs that are resting on the bench. This could potentially increase pressure beneath the thighs and possibly create tension at the hip joint. This tension has the potential to alter the angle of pelvic tilt and the alignment of the lumbar spine. 


\section{List of References}

[1] N. Sakai, "Hand pain attributed to overuse among professional pianists: a study of 200 cases," Medical Problems of Performing Artists, vol. 17, p. 178, Dec 2002.

[2] G. Comeau, ed., 10 Teachers' Viewpoints on Suzuki Piano, ch. F. Adams. Warner Brothers Publications, 1999.

[3] G. Comeau, ed., 10 Teachers' Viewpoints on Suzuki Piano, ch. B. Frest. Warner Brothers Publications, 1999.

[4] G. Comeau, ed., 10 Teachers' Viewpoints on Suzuki Piano, ch. C. Liccardo. Warner Brothers Publications, 1999.

[5] S. Fink, Mastering piano technique: A guide for students, teachers and performers. Oregon: Amadeus Press, 1992.

[6] T. Flash and F. Mussa-Ivaldi, "Human arm stiffness characteristics during the maintenance of posture," Experimental Brain Research, vol. 82, pp. 315-326, 1990.

[7] T. Tsuji, P. G. Morasso, K. Goto, and K. Ito, "Human hand impedance characteristics during maintained posture," Biological Cybernetics, vol. 72, pp. 475-485, 1995.

[8] H. Gomi and M. Kawato, "Human arm stiffness and equilibrium-point trajectory during multi-joint movement," Biological Cybernetics, vol. 76, pp. 163-171, 1997.

[9] D. Harding, K. Brandt, and B. Hillbury, "Minimization of finger joint forces and tendon tensions in pianists," Medical Problems of Performing Artists, vol. 4, pp. 103$108,1989$.

[10] H. Fry, "Overuse syndrome of the upper limb in musicians," Medical Journal of Australia, vol. 144, pp. 182-185, 1986.

[11] H. Fry, "The treatment of overuse injury syndrome," Maryland Medical Journal, vol. 42, pp. 277-282, 1993.

[12] H. Hoppman and N. Patrone, "A review of musculoskeletal problems in instrumentalist musicians," Seminars in Arthritis and Rheumatism, vol. 19, no. 117-126, 1989. 
[13] R. J. Lederman and L. H. Calabrese, "Overuse syndromes in instrumentalists," Medical Problems of Performing Artists, vol. 1, p. 7, Mar 1986.

[14] K. An, B. Hillberry, J. Ryu, and F. Bejjani, Current research in arts medicine, ch. Upper extremity biomechanics in musicians, pp. 139-146. Chicago: A Capella Books, 1993.

[15] H. Fry, "Incidence of overuse syndrome in the symphony ochestra," Medical Problems of Performing Artists, vol. 1, pp. 51-55, 1986.

[16] H. Fry, "Prevalence of overuse (injury) syndrome in australian music schools," British Journal of Industrial Medicine, vol. 44, pp. 35-40, 1987.

[17] F. Bejjani, G. Kaye, and M. Benham, "Musculoskeletal and neuromuscular conditions of instrumental musicians," Archives of Physical Medicine and Rehabilitation, vol. 77, no. 4, pp. 406-413, 1996.

[18] L. DeSmet, H. Ghyselen, and R. Lysens, "Incidence of overuse syndromes of the upper limb in young pianists and its correlation with hand size, hypermobility and playing habits.," Chirurgie de la Main, vol. 17, no. 4, pp. 309-313, 1998.

[19] M. Rosety-Rodriguez, F. O. nez, J. Farias, M. Rosety, C. Carrasco, A. Ribelles, J. Rosety, and M. G. del Valle, "The influence of the active range of movement of pianists' wrists on repetitive strain injury," European Journal of Anatomy, vol. 7, no. 2 , pp. $75-77,2003$.

[20] N. Shields and S. Dockrell, "The prevalence of injuries among pianists in music schools in ireland," Medical Problems of Performing Artists, vol. 15, p. 155, Dec 2000.

[21] J. Revak, "Incidence of upper extremity discomfort among piano students.," American Journal of Occupational Therapy, vol. 43, pp. 149-154, Mar 1989.

[22] D. Cayea and R. Manchester, "Instrument-specific rates of upper-extremity injuries in music students," Medical Problems of Performing Artists, vol. 13, p. 19, Mar 1998.

[23] A. G. Brandfonbrener, "Pathogenesis and prevention of problems of keyboardists," Medical Problems of Performing Artists, vol. 12, p. 57, Jun 1997.

[24] B. Webster and S. Snook, "The cost of compensable upper extremity cumulative trauma disorders.," Journal of Occupational Medicine, vol. 36, pp. 713-717, Jul 1994.

[25] K. Barber, ed., The Canadian Oxford Dictionary. Toronto: Oxford University Press, 1998.

[26] J. L. Young, "U.s. national cancer insititute surveillance, epidemiology and end results (seer) training web site." 
[27] H. Gray, Anatomy of the Human Body, 20th Edition. Philadelphia, PA: Lea and Febiger, 1918.

[28] T. McMahon, Muscles, Reflexes and Locomotion. Princeton University Press, 1984.

[29] O. Ortmann, The Physiological Mechanics of Piano Technique: An Experimental Study of the Nature of Muscular Action as Used in Piano Playing, and of the Effects Thereof Upon the Piano Key and the Piano Tone. EP Dutton and Co, 1929.

[30] N. Bernstein and T. Popova, "Study of the biodynamics of piano playing," Proceedings of the piano-methodological section of the State Institute of Music Science, vol. 1, pp. 5-47, 1930.

[31] R. R. A. I and A. S. I, "Orpheus wounded: The experience of pain in the professional worlds of the piano," Theory and Society (Historical Archive), vol. 25, pp. 1-44, Feb 1996.

[32] S. Lee, "Pianist's biomechanics, injuries and midi research.," Australian Journal of Music Education, vol. 1, pp. 30-37, 2001.

[33] I.-S. Chung, J. Ryu, N. Ohnishi, B. Rowen, and J. Headrich, "Wrist motion analysis in pianists," Medical Problems of Performing Artists, vol. 7, p. 1, Mar 1992.

[34] W. B. Meinke and F. Langendor, "Artsmedicine master class: Wrist tightness and pain in a young pianist," Medical Problems of Performing Artists, vol. 19, p. 97, Jun 2004.

[35] B. Wristen, "Fitness and form to reduce injuries," Clavier, vol. 35, no. 6, pp. 26-28, 1996.

[36] G. Sandor, On piano playing: motion, sound and expression. New York: Schirmer Books, 1981.

[37] A. Schultz, The riddle of the pianist's finger. New York: Carl Fischer, Inc., 1936.

[38] N. B. Reich and A. Burton, "Clara schumann: Old sources, new readings," The Musical Quarterly, vol. 70, no. 3, pp. 332-354, 1984.

[39] K.-N. An and F. Bejjani, "Analysis of upper-extremity performance in athletes and musicians," Hand Clinics, vol. 6, no. 3, pp. 393-403, 1990.

[40] J. Ryu, W. Cooney, L. Askew, K. An, and E. Chao, "Functional ranges of motion of the wrist joint," Journal of Hand Surgery [Am], vol. 16A, pp. 409-419, 1991.

[41] A. Grieco, E. Occhipinti, D. Colombini, O. Menoni, M. Bulgheroni, C. Frigo, and S. Boccardi, "Muscular effort and musculo-skeletal disorders in piano students: electromyographic, clinical and preventive aspects," Ergonomics, vol. 32, pp. 697-716, Jul 1989. 
[42] D. Harding, K. Brabdt, and B. Hillberry, "Finger joint force minimization in pianists using optimization techniques," Journal of Biomechanics, vol. 26, pp. 1403-1412, Dec 1993.

[43] B. G. Wristen, "Avoiding piano-related injury: A proposed theoretical procedure for biomechanical analysis of piano technique," Medical Problems of Performing Artists, vol. 15 , p. 55, Jun 2000.

[44] S. Brown, Medical problems of the instrumental musician, ch. Promoting a healthy keyboard technique. London: Martin Dunitz, 2000.

[45] F. G. Wolf, M. S. Keane, K. D. Brandt, and B. M. Hillberry, "An investigation of finger joint and tendon forces in experienced pianists," Medical Problems of Performing Artists, vol. 8, p. 84, Sep 1993.

[46] R. Hoppmann and R. Reid, "Musculoskeletal problems of performing artists.," Current Opinion in Rheumatology, vol. 7, pp. 147-150, Mar 1995.

[47] N. Sakai, M. C. Liu, F.-C. Su, A. T. Bishop, and K.-N. An, "Motion analysis of the fingers and wrist of the pianist," Medical Problems of Performing Artists, vol. 11, p. 24, Mar 1996.

[48] R. Smythe, "Up to speed," Music Teacher, vol. 76, pp. 14-15, Apr 1997.

[49] S.-H. Lee, "Pianists' hand ergonomics and touch control," Medical Problems of Performing Artists, vol. 5, p. 72, Jun 1990.

[50] J. Leijnse, "Anatomical factors predisposing to focal dystonia in the musician's hand-principles, theoretical examples, clinical significance.," Journal of Biomechanics, vol. 30, pp. 659-669, Jul 1997.

[51] G. Comeau, ed., 10 Teachers' Viewpoints on Suzuki Piano, ch. B. Schneiderman. Warner Brothers Publications, 1999.

[52] "Video 2: Forearm rotation." The Taubman Insistute of Piano, NY, 1995.

[53] S. Bernstein, Twenty Lessons in Keyboard Choreography. Seymour Bernstein Music, 1991.

[54] "Video 1: Introductory principles and concepts," 1995.

[55] T. Matthay, The visible and invisible in pianoforte technique: Being a digest of the author's technical teachings up to date. London: Oxford University Press, 1988 (Original work published in 1932; reprinted with corrections in 1947).

[56] P. Roës, Essai sur la technique du piano: le jeu de Chopin et de Liszt. Paris: Henry Lemoine, 1935. 
[57] W. Gieseking and K. Leimer, Piano technique: the shortest way to pianistic perfection. New York: Dover, 1972 (original work published in 1932).

[58] G. Comeau, ed., 10 Teachers' Viewpoints on Suzuki Piano, ch. M. Taggart. Warner Brothers Publications, 1999.

[59] G. Comeau, ed., 10 Teachers' Viewpoints on Suzuki Piano, ch. S. Williams. Warner Brothers Publications, 1999.

[60] G. Comeau, ed., 10 Teachers' Viewpoints on Suzuki Piano, ch. D. Harrel. Warner Brothers Publications, 1999.

[61] M. Deschaussées, Frédéric Chopin: 24 études, vers une interprétation. Paris: Éditions Van de Velde, 1995.

[62] T. Matthay, The act of tough in all its diversity: An analysis and synthesis of pianoforte tone-production. London: Longmass, Gree and Co., 1926.

[63] G. Comeau, ed., 10 Teachers' Viewpoints on Suzuki Piano, ch. M.C. Powell. Warner Brothers Publications, 1999.

[64] W. Gieseking and K. Leimer, Piano technique: rhythmics, dynamics, pedal and other problems of piano playing. New York: Dover, 1972 (Original work published in 1938).

[65] G. Comeau, ed., 10 Teachers' Viewpoints on Suzuki Piano, ch. M. Yurko. Warner Brothers Publications, 1999.

[66] G. Comeau, ed., 10 Teachers' Viewpoints on Suzuki Piano, ch. D. Koppelman. Warner Brothers Publications, 1999.

[67] E. Altenmüller, "From laetoli to carnegie: Evolution of the brain and hands as prerequisites of music performance in light of music physiology and neurobiology.," in Archaeology of early sound production and tonal scales. (E. Hickmann and R. Eichmann, eds.), pp. 9-34, Berlin: Publikationen des Deutschen Archäologischen Institutes, 2003.

[68] H.-C. Jabusch, "Movement analysis in pianists," in Music, Motor Control and the Brain (E. Altenmüller, M. Wiesendanger, and J. Kesselring, eds.), pp. 91-108, Oxford University Press, 2006.

[69] I. Hmelnitsky and N. Nettheim, "Weight-bearing manipulation: a neglected area of medical science relevant to piano playing and overuse syndrome.," Medical Hypotheses, vol. 23, pp. 209-217, Jun 1987.

[70] F. Bejjani, L. Ferrara, N. Xu, C. Tomaino, L. Pavlidis, J. Wu, and J. Dommerholt, "Comparison of three piano techniques as an implementation of a proposed experimental design," Medical Problems of Performing Artists, vol. 4, p. 109, Sep 1989. 
[71] E. J. Perrault, R. F. Kirsch, and A. M. Acosta, "Multiple-input, multiple-output system identification for characterization of limb stiffness dynamics," Biological Cybernetics, vol. 80, pp. 327-337, 1999.

[72] D. Jindrich, A. Balakrishnan, and J. Dennerlein, "Finger joint impedance during tapping on a computer keyswitch.," Journal of Biomechanics, vol. 37, pp. 1589-1596, Oct 2004.

[73] P. L. Gribble, L. I. Mullin, N. Cothros, and A. Mattar, "Role of cocontraction in arm movement accuracy," Journal of Neurophysiology, vol. 89, pp. 2396-2405, 2003.

[74] R. Osu, D. W. Franklin, H. Kato, H. Gomi, K. Domen, T. Yoshioka, and M. Kawato, "Short- and long-term changes in joint co-contraction associated with motor learning as revealed from surface emg," Journal of Neurophysiology, vol. 88, pp. 991-1004, 2002 .

[75] I. Arvidson, E. Eriksson, and M. Pitman, Rehabilitation of the Injured Knee, ch. Neuromuscular basis of rehabilitation, pp. 210-234. St Louis: C V Mosby, 1984.

[76] C. E. English, Stiffness Behaviour in Two Degree of Freedom Mechanisms. PhD thesis, Carleton University, 1999.

[77] T. Mark, What Every Pianist Needs to Know About the Body. Chicago: GIA Publications, 2004.

[78] M. McCray, "Harmful practices that cause injuries," Clavier, vol. 33, pp. 13-18, 1994.

[79] A. Fraser, The Craft of Piano Playing. The Scarecrow Press, Inc, 2003.

[80] H. Kataoka, "The piano method that allows anybody to be able to play well," Suzuki Piano Basics Foundation News, vol. 9.3, 2004.

[81] M. S. Lucas, "A history of piano pedagogy in the united states from 1780," Master's thesis, University of Nebraska, Illinois, 1948.

[82] G. Comeau, ed., 10 Teachers' Viewpoints on Suzuki Piano. Warner Brothers Publications, 1999.

[83] R. Brockman, P. Chamagne, and R. Tubiana, "The upper extremity in musicians," Chapter 59 In: The Hand, pp. 873-885, 1993.

[84] W. Kapit, R. I. Macey, and E. Meisami, The Physiology Coloring Book. San Francisco: Benjamin Cumming Science Publishing, 2nd edition ed., 2000.

[85] A. Freivalds, Biomechanics of the upper limbs: Mechanics, modeling and musculoskeletal injuries. CRC Press, 2004. 
[86] J. Rothwell, K. Higuchi, and J. Obeso, "The offset cortical potential: and electrical correlate of movement inhibition in man," Movement Disorders, vol. 13, pp. 330-335, 1998.

[87] M. Lee, D. Kelly, and G. Steven, "A model of spine, ribcage and pelvic responses to a specific lumbar manipulative force in relaxed subjects," Journal of Biomechanics, vol. 28, no. 11, pp. 1403-1408, 1995.

[88] D. H. Peterson and T. F. Bergmann, Chiropractic Technique, 2nd Edition, ch. Chapter 2: Joint Anatomy and Basic Biomechanics, pp. 11-38. Mosby, 2002.

[89] L. H. Phillip, Piano technique tone, touch, phrasing and dynamics. New York: Dover Publications Inc., 1969.

[90] H. Jongen, J. van der Gon Denier, and C. Gielen, "Inhomogeneous activation of motoneurone pools as revealed by co-contraction of antagonistic human arm muscles," Experimental Brain Research, vol. 75, no. 3, pp. 555-562, 1989.

[91] T. E. Milner, "Contribution of geometry and joint stiffness to mechanical stability of the human arm," Experimental Brain Research, vol. 143, no. 4, pp. 515-519, 2002.

[92] P. L. Gribble and D. J. Ostry, "Independent coactivation of shoulder and elbow muscles," Experimental Brain Research, vol. 123, pp. 355-360, 1998.

[93] M. Suzuki, D. M. Shiller, P. L. Gribble, and D. J. Ostry, "Relationship between cocontraction, movement kinematics and phasic muscle activity in single-joint arm movement," Experimental Brain Research, vol. 140, pp. 171-181, September 2001.

[94] B. Day, C. Mardsen, J. Obeso, and J. Rothwell, "Reciprocal inhibition between the muscles of the human forearm," Journal of Physiology, vol. 349, pp. 519-534, 1984.

[95] C. Crone, "Reciprocal inhibition in man," Danish Medical Bulletin, vol. 40, pp. 571581, Nov 1993.

[96] P. Morasso, "Spatial control of arm movements," Experimental Brain Research, vol. 42 , pp. 223-227, 1981.

[97] R. Osu and H. Gomi, "Multijoint muscle regulation mechanisms examined by measured human arm stiffness and emg signals," The American Physiological Society, vol. 81, pp. 1458-1468, April 1999.

[98] J. Dolan, M. Friedman, and M. Nagurka, "Dynamic and loaded impedance components int he maintenance of human arm posture," IEEE Transactions on Systems, Man and Cybernetics, vol. 23, pp. 698-709, 1993. 
[99] J. MacNeil, R. Kearney, and I. Hunter, "Identification of time-varying biological systems from ensemble data," IEEE Transactions on Biomedical Engineering, vol. 39, pp. 1213-1225, 1992.

[100] R. Kirsch and R. Kearney, "Identification of time-varying stiffness dynamics of the human ankle joint during an imposed movement," Experimental Brain Research, vol. 114 , pp. $71-85,1997$.

[101] M. L. Latash, "Reconstruction of equilibrium trajectories and joint stiffness patterns during single-joint voluntary movements under different instructions," Biological Cybernetics, vol. 71, pp. 441-450, 1994.

[102] D. M. Corcos, H.-Y. Jian, J. Wilding, and G. L. Gottlieb, "Fatigue induced changes in phasic muscle activation patterns for fast elbow flexion movements," Experimental Brain Research, vol. 142, pp. 1-12, 2002.

[103] J. McIntyre, F. Mussa-Ivaldi, and E. Bizzi, "The control of stable postures in the multi-joint arm," Experimental Brain Research, vol. 110, pp. 248-264, 1996.

[104] L.-Q. Zhang and W. Z. Rymer, "Simultaneous and nonlinear identification of mechanical and reflex properties of human elbow joint muscles," IEEE Transactions on Biomedical Engineering, vol. 44, no. 12, pp. 1192-1209, 1997.

[105] D. A. Winter, Biomechanics and motor control of human movement, 3rd Edition. Wiley, 2004.

[106] D. Parlitz, T. Peschel, and E. Altenmüller, "Assessment of dynamic finger forces in pianists: effects of training and expertise.," Journal of Biomechanics, vol. 31, pp. 1063-1067, Nov 1998. 


\section{Appendix A}

\section{Creation of the Connector Block}

This appendix contains the methods and drawings for the creation of the connector block that was used to attach the Phantom to the forearm.

The block was created using a rapid prototyping machine, the Dimension ${ }^{1}$. The machine converts a three-dimensional drawing in .STL format into a three-dimensional model made of ABS plastic by printing with thin layers of the plastic and binding them together in the specified shape.

The Phantom Omni's stylus is removable to allow tip replacement for other applications. The connector on the arm is a $1 / 4$ " phono plug. A $1 / 4$ " stereo panel-mount audio jack was used in conjunction with the ABS block to connect the Phantom to the subject's right forearm.

The audio jack was bolted to the block through the $1 / 8$ " panel. The Phantom's phono plug entered through the hole with three notches, aligning the notch on the Phantom with the vertical notch on the block; This is the end of the block to be placed distally on the forearm. The phono plug clicked into the jack, housed in the hollow section at the opposite end of the block; this end of the block will be placed proximally on the forearm. The block was designed to be raised $1.5 \mathrm{~cm}$ off the forearm to allow manoeuvring room for the Phantom Omni's arm.

\footnotetext{
${ }^{1}$ Computer-Aided Products, Inc., Peabody, MA
} 
The block is positioned on the subject's forearm, just proximal to the wrist to allow for full wrist extension. It is positioned so that it runs lengthwise along the arm, with the Phantom connected through the distal end and the stereo jack at the proximal end.

Detailed drawings of the block follow. 


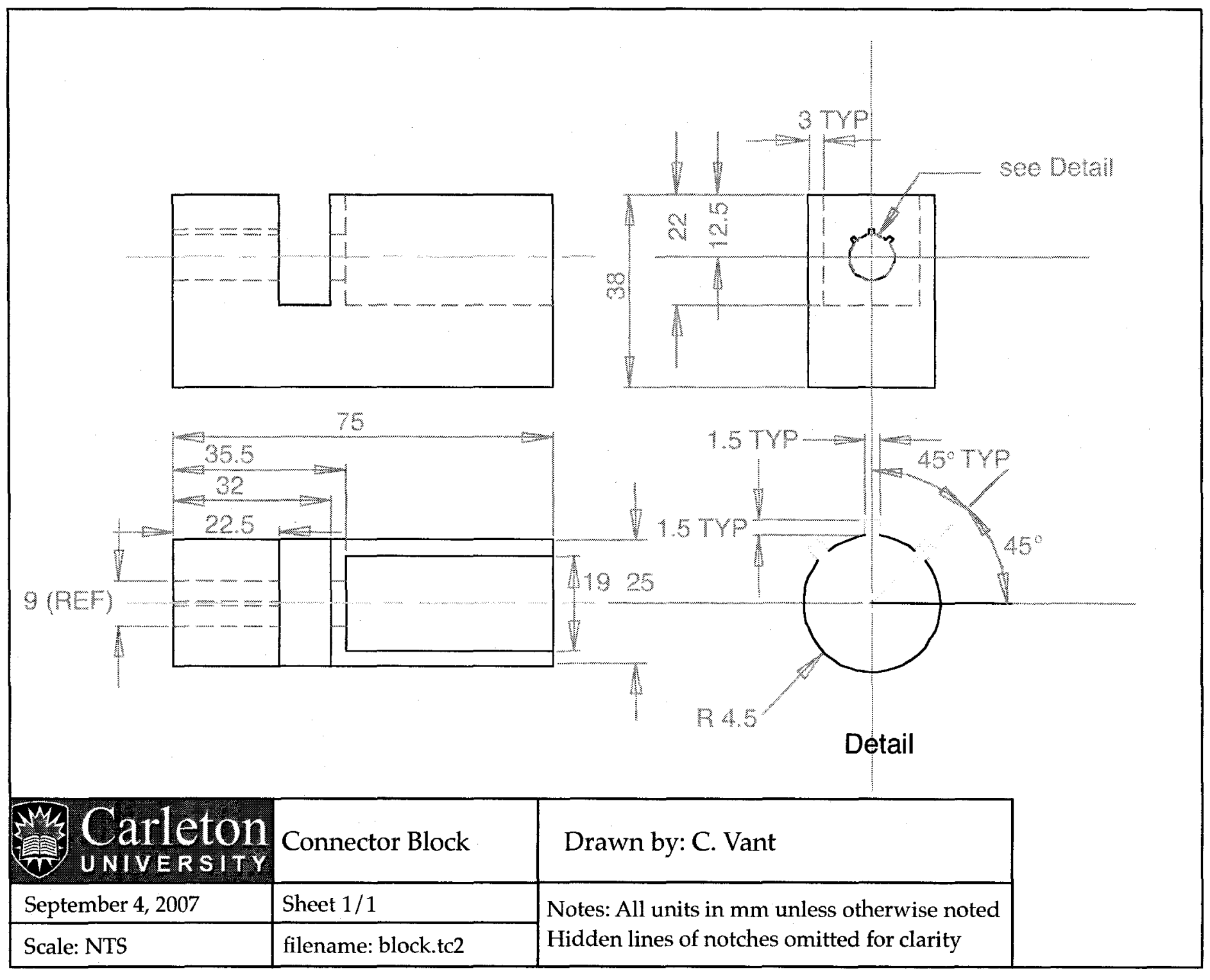




\section{Appendix B}

\section{Music}

This appendix contains two copies of the music composed by Ann Southam for the purpose of these experiments. The first copy is the music as presented to the subjects during the experiment. The second copy is marked with circles and notes that indicate which notes triggered perturbations.

Subjects were asked to sight-read this music on the Yamaha Disklavier. Software was written to create trigger notes throughout the piece. These cause a perturbation from the Phantom Omni to be applied to the subject's wrist during playing. 

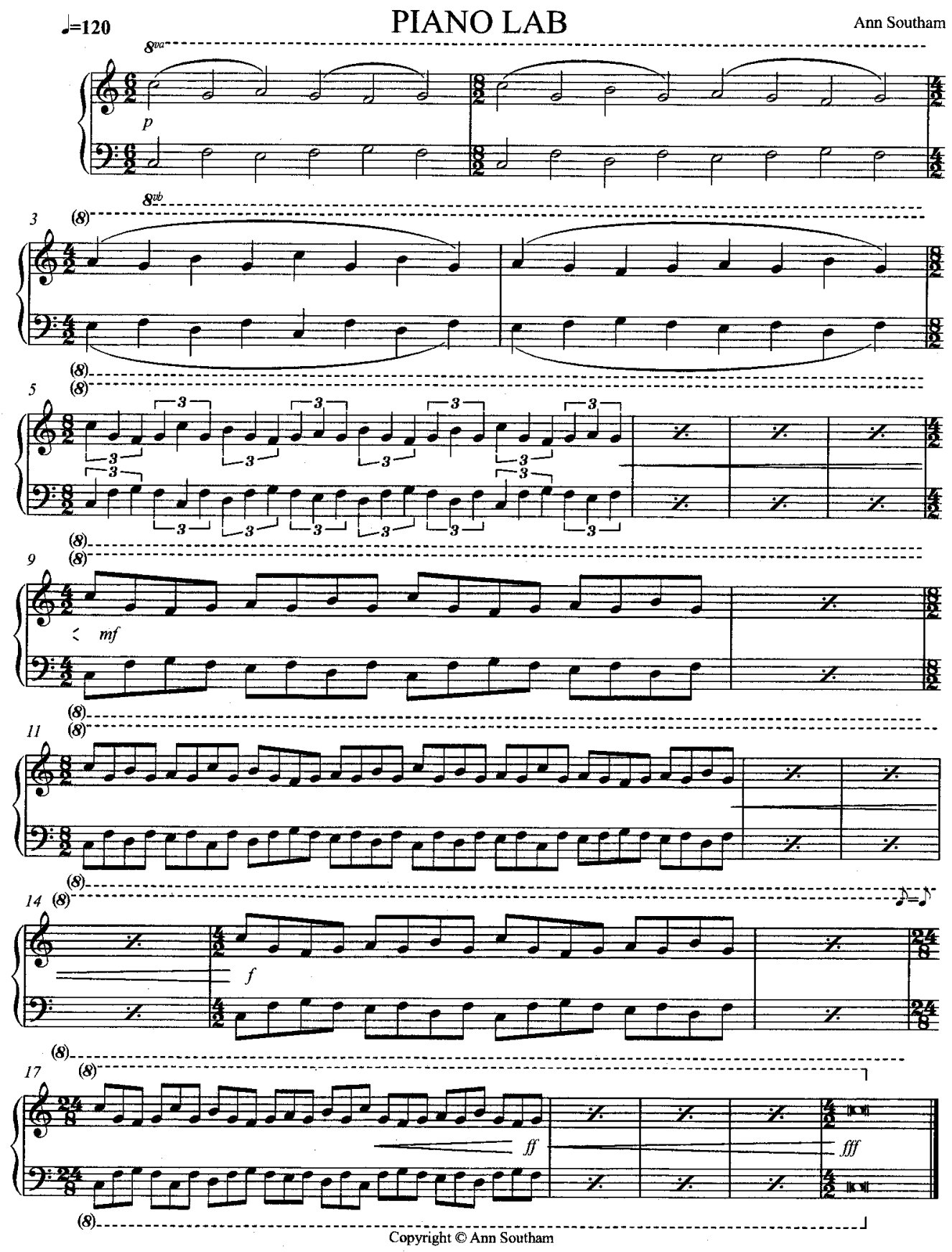

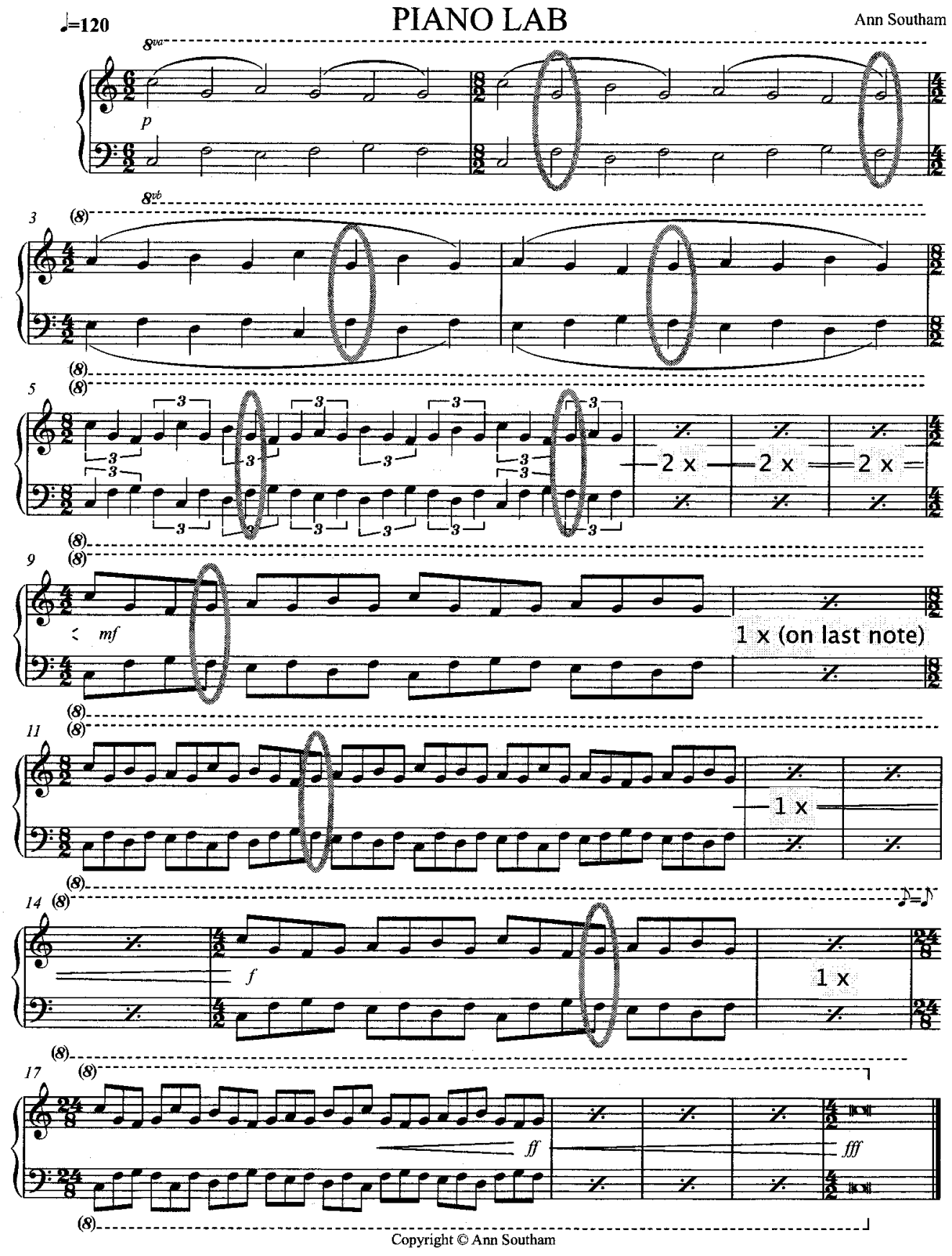


\section{Appendix C}

\section{Code Listing 1: Software for the Experiment}

This appendix contains the code listing for the experimental software used to trigger perturbations from the Phantom Omni using the Yamaha Disklavier at the Piano Pedagogy Research Laboratory at the University of Ottawa. It collects and records MIDI data from the Disklavier, which provides a quantitative analysis of the piano player's performance, as well as force and position data from the Phantom Omni, which provides information about the haptic device's perturbation and the piano player's response to the perturbation.

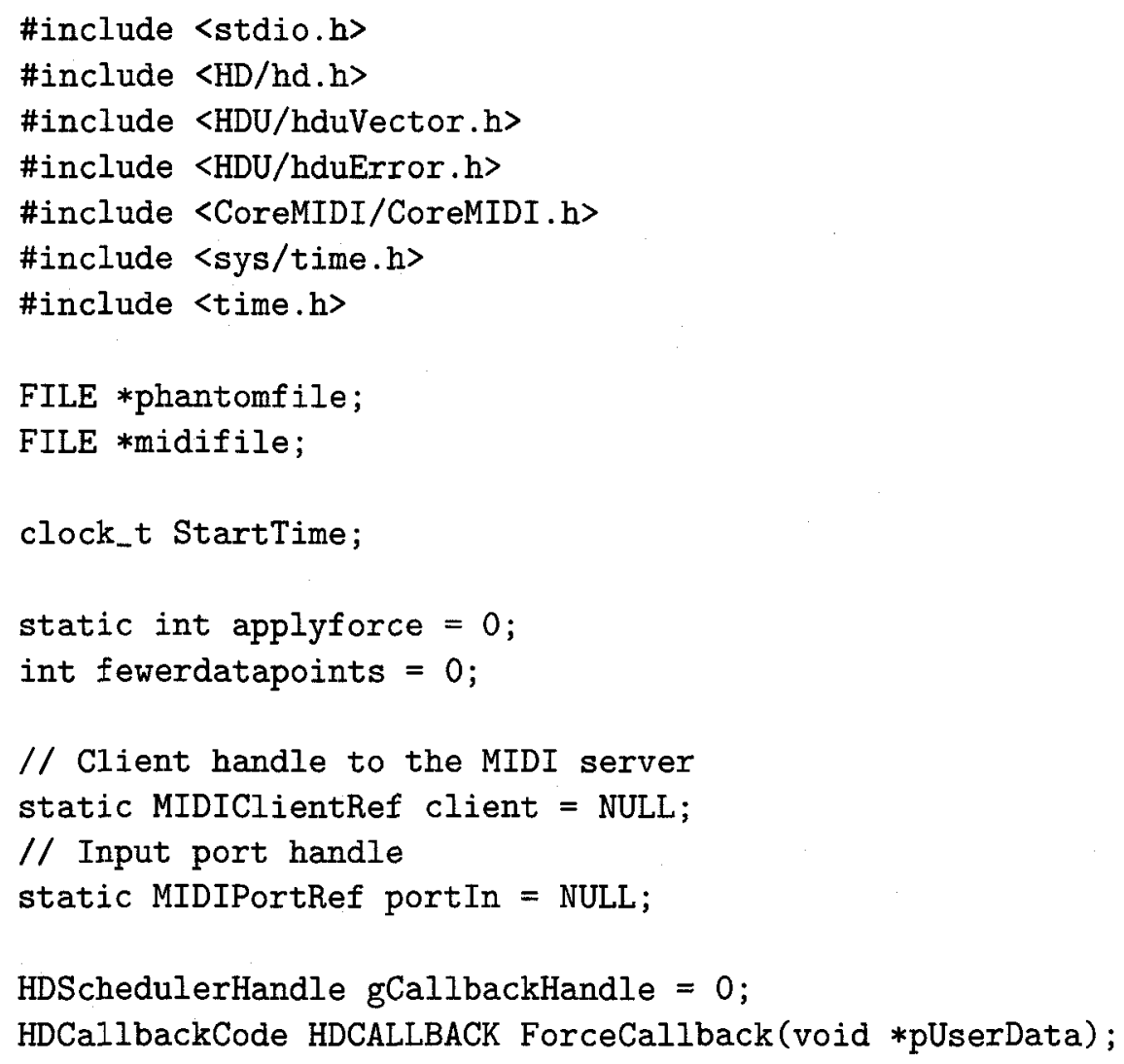




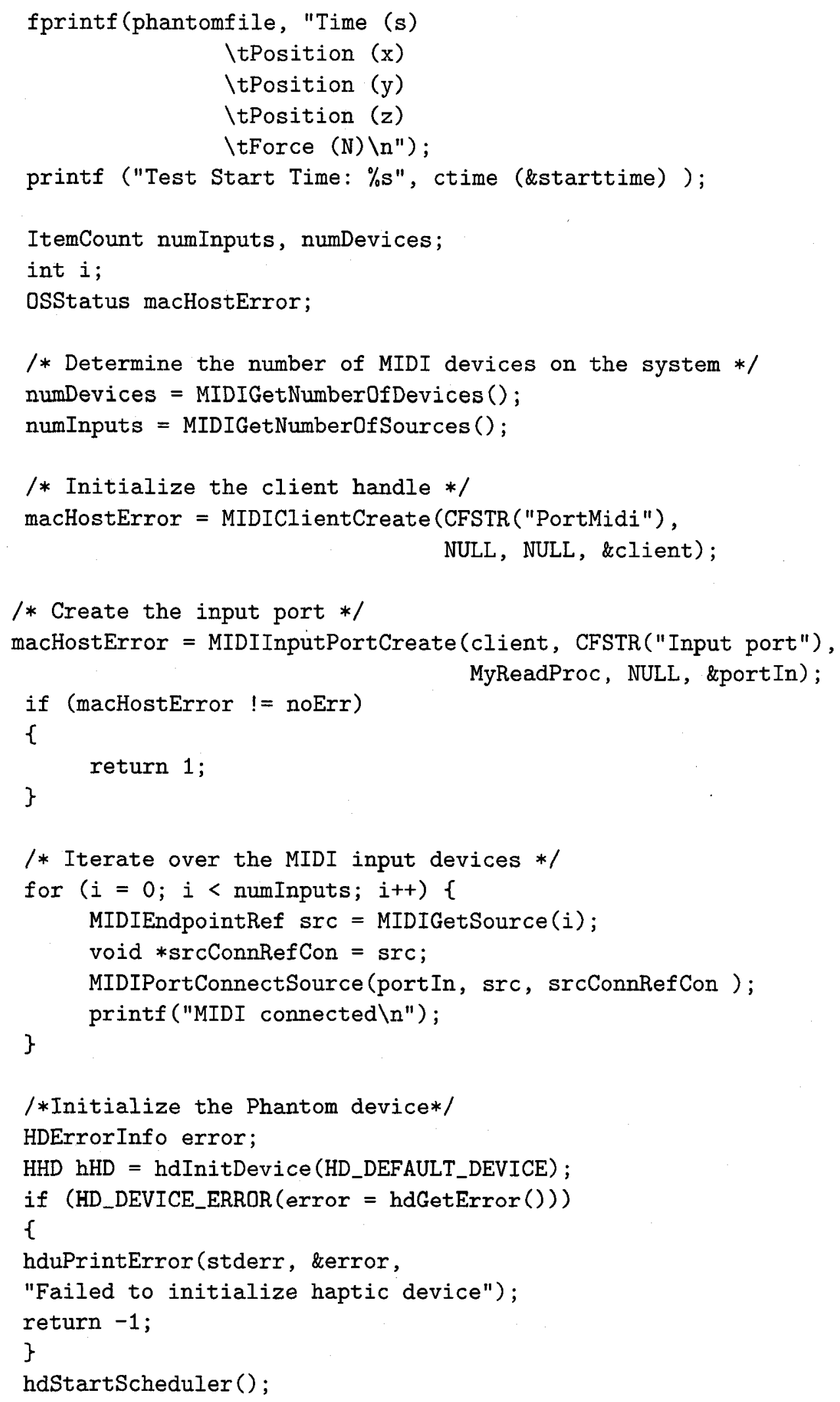




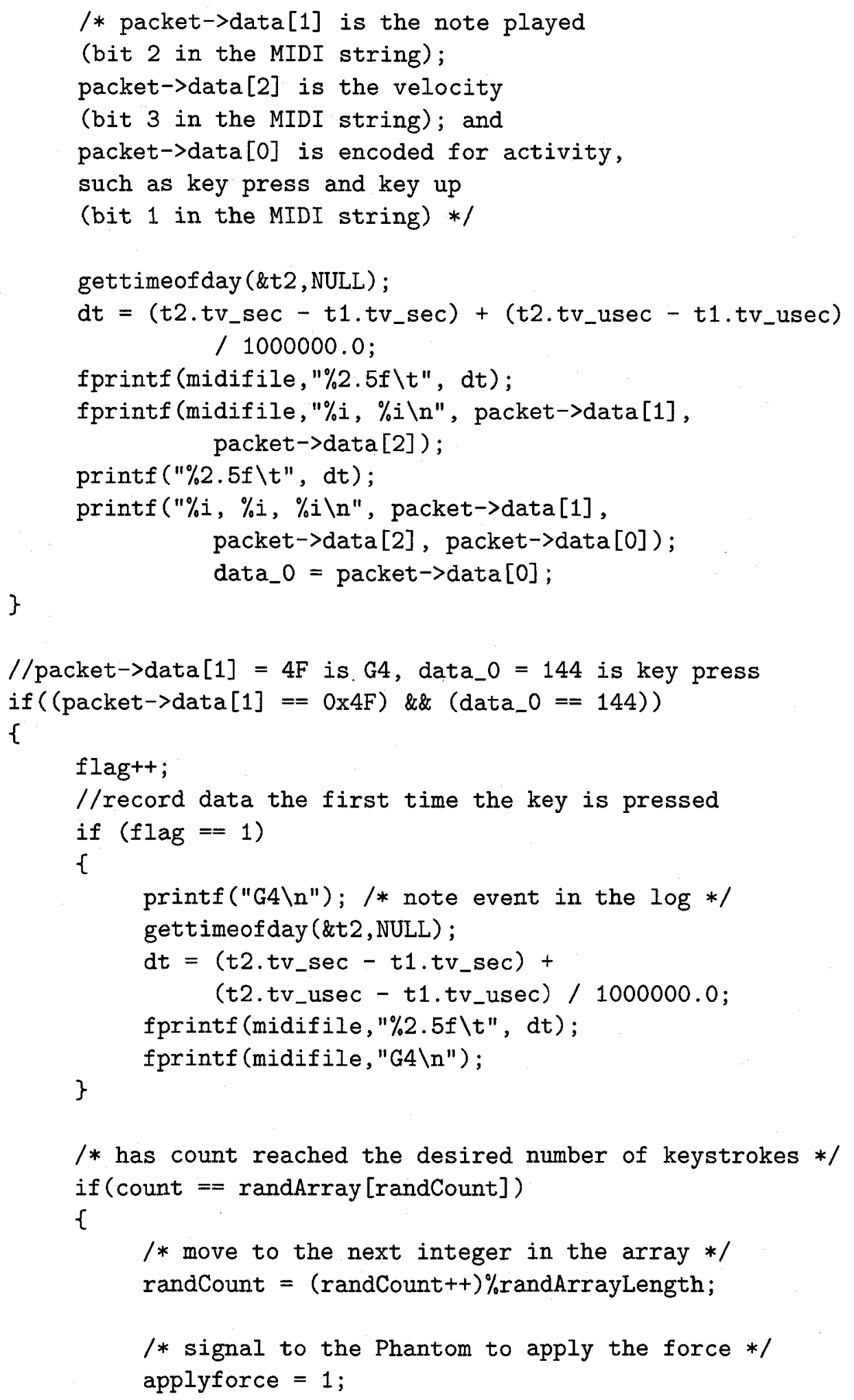




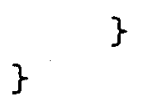




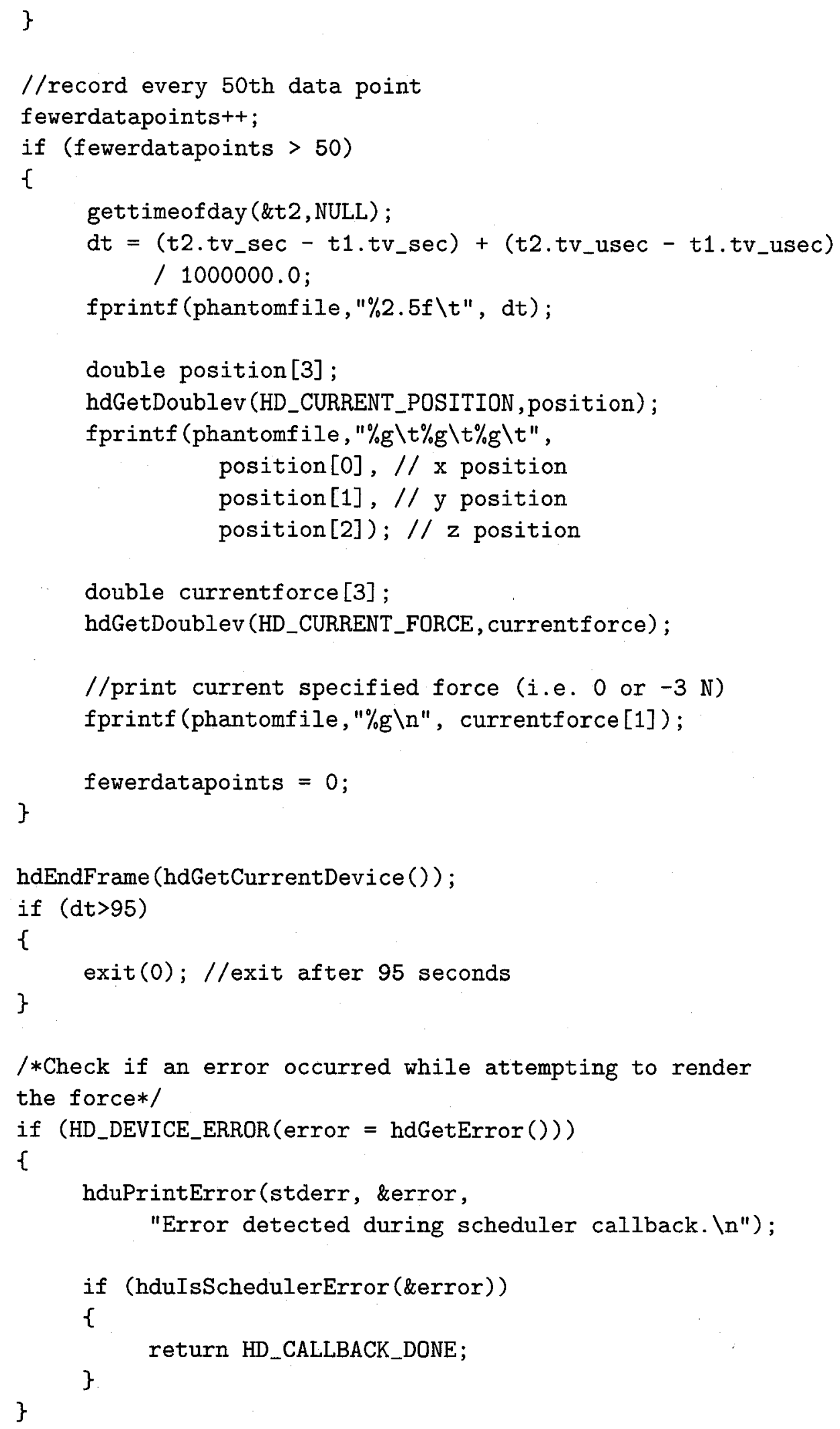


\}

return HD_CALLBACK_CONTINUE; 


\section{Appendix D}

\section{Mann-Whitney U Test}

This appendix contains the results of the statistical testing to determine if the perturbation had a significant effect on wrist height. Two population groups were used. The first compared perturbed and unperturbed values of the change in wrist height, that is the difference between maximum and minimum peak values for similar notes (Tables D.1 and D.2). The second group compared perturbed and unperturbed values of the actual wrist height at the 'note off' signal for similar notes. For subject 1 in bars $1-4$, only the results from the half notes are included; due to the oscillatory pattern of movement quarter notes and half notes could not be included in the same analysis.

The Mann-Whitney U Test is a non-parametric, distribution-free test. The variables to perform the test are defined below. The validity of a directional test must be verified; for $\Delta h, \boldsymbol{R}_{\text {ave1 }}>\boldsymbol{R}_{\text {ave2 }}$ and for $\boldsymbol{h}, \boldsymbol{R}_{1}<\boldsymbol{R}_{2}$. Find $\boldsymbol{U}_{\text {critical }}$ for the $\left(\boldsymbol{n}_{1}, \boldsymbol{n}_{2}\right)$ pair from tables

1. Sample size $\left(\boldsymbol{n}_{\boldsymbol{i}}\right)$

2. Summed ranks for each sample $\left(\boldsymbol{R}_{\boldsymbol{i}}\right)$

3. $U_{i}$

$$
U_{1}=n_{1} n_{2}+\frac{n_{1}\left(n_{1}+1\right)}{2}
$$

The conclusions from Table D.1 are that in a directional test there is evidence that the perturbation increases the maximum-minimum peak height at the 0.01 level of significance (99\% confidence). 
The conclusions from Table D.2 are that in a directional test there is evidence that the perturbation increases the maximum-minimum peak height at the 0.01 level of significance (99\% confidence).

The conclusions from Table D. 3 are that in a directional test there is evidence that the perturbation decreases the actual wrist height at the 'note off' signal at the 0.01 level of significance ( $99 \%$ confidence).

The conclusions from Table D.4 are that in a directional test there is evidence that the perturbation decreases the actual wrist height at the 'note off' signal at the 0.01 level of significance ( $99 \%$ confidence).

The conclusions from Table D.5 are that in a directional test there is evidence that the perturbation decreases the actual wrist height at the 'note off' signal at the 0.01 level of significance ( $99 \%$ confidence).

The conclusions from Table D.6 are that in a directional test there is evidence that the perturbation decreases the actual wrist height at the 'note off' signal at the 0.01 level of significance ( $99 \%$ confidence).

The conclusions from Table D.7 are that in a directional test there is evidence that the perturbation decreases the actual wrist height at the 'note off' signal at the 0.01 level of significance ( $99 \%$ confidence).

The conclusions from Table D. 8 are that in a directional test there is evidence that the perturbation decreases the actual wrist height at the 'note off' signal at the 0.05 level of significance ( $99 \%$ confidence). 
Table D.1: Statistical analysis for change in wrist height for subject 1, bars 1-4

\begin{tabular}{|c|c|c|c|c|}
\hline & \multicolumn{2}{|c|}{ Unperturbed } & \multicolumn{2}{|c|}{ Perturbed } \\
\hline & $\Delta h(\mathrm{~mm})$ & Rank & $\Delta h(\mathrm{~mm})$ & Rank \\
\hline & 10.21 & 1 & & \\
\hline & 12.73 & 2 & & \\
\hline & 13.60 & 3 & & \\
\hline & 15.37 & 4 & & \\
\hline & 20.12 & 5 & & \\
\hline & 29.38 & 6 & & \\
\hline & 35.88 & 7 & & \\
\hline & 36.27 & 8 & & \\
\hline & 40.70 & 9 & & \\
\hline & 45.65 & 10 & & \\
\hline & & & 45.66 & 11 \\
\hline & 49.49 & 12 & & \\
\hline & & & 49.77 & 13 \\
\hline & 53.59 & 14 & & \\
\hline & & & 59.81 & 15 \\
\hline & & & 64.85 & 16 \\
\hline & & & 68.19 & 17 \\
\hline & & & 74.81 & 18 \\
\hline & & & 79.14 & 19 \\
\hline & & & 93.25 & 20 \\
\hline$n_{i}$ & 12 & & 8 & \\
\hline$n_{t o t a l}$ & 20 & & & \\
\hline $\boldsymbol{R}_{i}$ & & 81 & & 129 \\
\hline $\boldsymbol{R}_{a v e}$ & & 6.75 & & 16.13 \\
\hline$U_{i}$ & 93 & & 3 & \\
\hline$U_{\min }$ & 3 & & & \\
\hline
\end{tabular}


Table D.2: Statistical analysis for change in wrist height for subject 2, bars 1-4

\begin{tabular}{|c|c|c|c|c|}
\hline & \multicolumn{2}{|c|}{ Unperturbed } & \multicolumn{2}{|c|}{ Perturbed } \\
\hline & $\Delta h(\mathrm{~mm})$ & Rank & $\Delta h(\mathrm{~mm})$ & Rank \\
\hline & 0 & 1 & & \\
\hline & 2.26 & 2 & & \\
\hline & 2.69 & 3 & & \\
\hline & 4.15 & 4 & & \\
\hline & 5.62 & 5 & & \\
\hline & 7.17 & 6 & & \\
\hline & 7.92 & 7 & & \\
\hline & & & 11.49 & 8 \\
\hline & 12.37 & 9 & & \\
\hline & 12.96 & 10 & & \\
\hline & & & 13.82 & 11 \\
\hline & & & 15.91 & 12 \\
\hline & & & 23.02 & 13 \\
\hline & & & 26.04 & 14 \\
\hline & & & 31.47 & 15 \\
\hline$n_{i}$ & 9 & & 6 & \\
\hline$n_{t o t a l}$ & 15 & & & \\
\hline$R_{i}$ & & 47 & & 73 \\
\hline$R_{a v e}$ & & 5.22 & & 12.17 \\
\hline$U_{i}$ & 52 & & 2 & \\
\hline$U_{\min }$ & 2 & & & \\
\hline
\end{tabular}


Table D.3: Statistical analysis for actual wrist height at 'note off' for subject 1, bars 1-4

\begin{tabular}{|c|c|c|c|c|}
\hline & \multicolumn{2}{|c|}{ Unperturbed } & \multicolumn{2}{|c|}{ Perturbed } \\
\hline & $\Delta h(\mathrm{~mm})$ & Rank & $\Delta h(\mathrm{~mm})$ & Rank \\
\hline & & & -20.04 & 1 \\
\hline & & & -19.14 & 2 \\
\hline & & & -13.06 & 3 \\
\hline & & & -11.63 & 4 \\
\hline & & & -8.19 & 5 \\
\hline & & & -5.67 & 6 \\
\hline & & & -4.27 & 7 \\
\hline & & & 8.87 & 8 \\
\hline & 20.16 & 9 & & \\
\hline & 20.75 & 10 & & \\
\hline & 22.44 & 11 & & \\
\hline & 23.08 & 12 & & \\
\hline & 24.55 & 13 & & \\
\hline & 28.58 & 14 & & \\
\hline & 29.97 & 15 & & \\
\hline & 35.45 & 16 & & \\
\hline & 37.91 & 17 & & \\
\hline & 43.18 & 18 & & \\
\hline & 51.19 & 19 & & \\
\hline & 67.85 & 20 & & \\
\hline$n_{i}$ & 12 & & 8 & \\
\hline$n_{\text {total }}$ & 20 & & & \\
\hline $\boldsymbol{R}_{i}$ & & 174 & & 36 \\
\hline $\boldsymbol{R}_{\text {ave }}$ & & 14.5 & & 4.5 \\
\hline$U_{i}$ & 0 & & 96 & \\
\hline$U_{\min }$ & 0 & & & \\
\hline
\end{tabular}


Table D.4: Statistical analysis for actual wrist height at 'note off' for subject 2, bars 1-4

\begin{tabular}{|c|c|c|c|c|}
\hline & \multicolumn{2}{|c|}{ Unperturbed } & \multicolumn{2}{|c|}{ Perturbed } \\
\hline & $\Delta \boldsymbol{h}(\mathrm{mm})$ & Rank & $\Delta h(\mathrm{~mm})$ & Rank \\
\hline & & & 4.16 & 1 \\
\hline & & & 6.53 & 2 \\
\hline & & & 6.84 & 3 \\
\hline & & & 8.58 & 4 \\
\hline & & & 8.81 & 5 \\
\hline & & & 11.99 & 6 \\
\hline & & & 13.31 & 7 \\
\hline & & & 13.33 & 8 \\
\hline & & & 14.52 & 9 \\
\hline & & & 15.44 & 10 \\
\hline & & & 18.58 & 11 \\
\hline & & & 19.26 & 12 \\
\hline & 20.48 & 13 & & \\
\hline & 21.80 & 14 & & \\
\hline & 23.61 & 15 & & \\
\hline & 23.69 & 16 & & \\
\hline & 24.52 & 17 & & \\
\hline & 24.64 & 18 & & \\
\hline & 25.23 & 19 & & \\
\hline & $\vdots$ & $\vdots$ & & \\
\hline & 33.75 & 32 & & \\
\hline$n_{i}$ & 20 & & 12 & \\
\hline$n_{\text {total }}$ & 32 & & & \\
\hline $\boldsymbol{R}_{i}$ & & 450 & & 78 \\
\hline $\boldsymbol{R}_{\text {ave }}$ & & 22.5 & & 6.5 \\
\hline$U_{i}$ & 0 & & 240 & \\
\hline$U_{\min }$ & 0 & & & \\
\hline$U_{\text {criti }}$ & $=60$ for & $=0.0$ & 7 for $p=$ & .05 \\
\hline
\end{tabular}


Table D.5: Statistical analysis for actual wrist height at 'note off' for subject 1, perturbation 5 , bars $5-8$

\begin{tabular}{|c|c|c|c|c|}
\hline & \multicolumn{2}{|c|}{ Unperturbed } & \multicolumn{2}{|c|}{ Perturbed } \\
\hline & $\Delta h(\mathrm{~mm})$ & Rank & $\Delta h(\mathrm{~mm})$ & Rank \\
\hline & & & -10.51 & 1 \\
\hline & & & -7.44 & 2 \\
\hline & & & -4.90 & 3 \\
\hline & & & -4.34 & 4 \\
\hline & & & -3.19 & 5 \\
\hline & & & 0.06 & 6 \\
\hline & & & 6.82 & 7 \\
\hline & & & 9.32 & 8 \\
\hline & 9.41 & 9 & & \\
\hline & 11.83 & 10 & & \\
\hline & 14.30 & 11 & & \\
\hline & 14.62 & 12 & & \\
\hline & 16.26 & 13 & & \\
\hline & 17.97 & 14 & & \\
\hline & 18.99 & 15 & & \\
\hline & 19.17 & 16 & & \\
\hline & 19.74 & 17 & & \\
\hline & 19.93 & 18 & & \\
\hline & 20.64 & 19 & & \\
\hline & 21.77 & 20 & & \\
\hline & $\vdots$ & $\vdots$ & & \\
\hline & 31.18 & 28 & & \\
\hline$n_{i}$ & 20 & & 8 & \\
\hline$n_{\text {total }}$ & 28 & & & \\
\hline$R_{i}$ & & 370 & & 36 \\
\hline $\boldsymbol{R}_{\text {ave }}$ & & 18.5 & & 4.5 \\
\hline$U_{i}$ & 0 & & 160 & \\
\hline$U_{\min }$ & 0 & & & \\
\hline$U_{\text {crit }}$ & $=34$ for & $=0.01$ & 7 for $p=$ & 05 \\
\hline
\end{tabular}


Table D.6: Statistical analysis for actual wrist height at 'note off' for subject 1, perturbation 6 , bars $5-8$

\begin{tabular}{|c|c|c|c|c|}
\hline & \multicolumn{2}{|c|}{ Unperturbed } & \multicolumn{2}{|c|}{ Perturbed } \\
\hline & $\Delta h(\mathrm{~mm})$ & Rank & $\Delta \boldsymbol{h}(\mathrm{mm})$ & Rank \\
\hline & & & 11.12 & 1 \\
\hline & & & 12.02 & 2 \\
\hline & & & 14.82 & 3 \\
\hline & & & 15.83 & 4 \\
\hline & & & 17.65 & 5 \\
\hline & & & 18.41 & 6 \\
\hline & & & 22.62 & 7 \\
\hline & & & 28.84 & 8 \\
\hline & 37.34 & 9 & & \\
\hline & 40.12 & 10 & & \\
\hline & 40.39 & 11 & & \\
\hline & 40.63 & 12 & & \\
\hline & 40.67 & 13 & & \\
\hline & 41.86 & 14 & & \\
\hline & 41.93 & 15 & & \\
\hline & 41.98 & 16 & & \\
\hline & 42.20 & 17 & & \\
\hline & 43.02 & 18 & & \\
\hline & 43.46 & 19 & & \\
\hline & 44.05 & 20 & & \\
\hline & $\vdots$ & $\vdots$ & & \\
\hline & 48.84 & 28 & & \\
\hline$n_{i}$ & 20 & & 8 & \\
\hline$n_{t o t a l}$ & 28 & & & \\
\hline $\boldsymbol{R}_{i}$ & & 370 & & 36 \\
\hline $\boldsymbol{R}_{\text {ave }}$ & & 18.5 & & 4.5 \\
\hline$U_{i}$ & 0 & & 160 & \\
\hline$U_{\min }$ & 0 & & & \\
\hline$U_{\text {crit }}$ & 34 for & $=0.0$ & 7 for $p$ & \\
\hline
\end{tabular}


Table D.7: Statistical analysis for actual wrist height at 'note off' for subject 2, perturbation 5 , bars 5-8

\begin{tabular}{|c|c|c|c|c|}
\hline & \multicolumn{2}{|c|}{ Unperturbed } & \multicolumn{2}{|c|}{ Perturbed } \\
\hline & $\Delta h(\mathrm{~mm})$ & Rank & $\Delta h(\mathrm{~mm})$ & Rank \\
\hline & & & 7.26 & 1 \\
\hline & & & 10.45 & 2 \\
\hline & & & 11.40 & 3 \\
\hline & & & 11.42 & 4 \\
\hline & & & 14.49 & 5 \\
\hline & & & 21.95 & 6 \\
\hline & 24.30 & 7 & & \\
\hline & 24.84 & 8 & & \\
\hline & & & 24.88 & 9 \\
\hline & 25.44 & 10 & & \\
\hline & & & 25.74 & 11 \\
\hline & 26.44 & 12 & & \\
\hline & 26.84 & 13 & & \\
\hline & 27.22 & 14 & & \\
\hline & 27.82 & 15 & & \\
\hline & 27.90 & 16 & & \\
\hline & 28.96 & 17 & & \\
\hline & 30.88 & 18 & & \\
\hline & 30.91 & 19 & & \\
\hline & 32.15 & 20 & & \\
\hline & 40.27 & 28 & & \\
\hline$n_{i}$ & 20 & & 8 & \\
\hline$n_{\text {total }}$ & 28 & & & \\
\hline $\boldsymbol{R}_{i}$ & & 365 & & 41 \\
\hline$R_{a v e}$ & & 18.25 & & 5.13 \\
\hline$U_{i}$ & 5 & & 155 & \\
\hline$U_{\min }$ & 5 & & & \\
\hline$U_{c r i}$ & $f_{0}$ & o & for $\mathrm{n}$ & .05 \\
\hline
\end{tabular}


Table D.8: Statistical analysis for actual wrist height at 'note off' for subject 2, perturbation 6 , bars $5-8$

\begin{tabular}{|c|c|c|c|c|}
\hline & \multicolumn{2}{|c|}{ Unperturbed } & \multicolumn{2}{|c|}{ Perturbed } \\
\hline & $\Delta h(\mathrm{~mm})$ & Rank & $\Delta h(\mathrm{~mm})$ & Rank \\
\hline & & & 11.09 & 1 \\
\hline & & & 13.15 & 2 \\
\hline & & & 13.61 & 3 \\
\hline & & & 22.02 & 4 \\
\hline & 26.99 & 5 & & \\
\hline & 27.45 & 6 & & \\
\hline & 27.58 & 7 & & \\
\hline & 28.04 & 8 & & \\
\hline & 28.24 & 9 & & \\
\hline & & & 29.42 & 10 \\
\hline & 31.83 & 11 & & \\
\hline & 33.17 & 12 & & \\
\hline & 33.24 & 13 & & \\
\hline & & & 34.36 & 14 \\
\hline & 34.39 & 15 & & \\
\hline & $\vdots$ & 曈 & & \\
\hline & 37.81 & 19 & & \\
\hline & & & 39.65 & 20 \\
\hline & & & 39.92 & 21 \\
\hline & 40.15 & 22 & & \\
\hline & $\vdots$ & $\vdots$ & & \\
\hline & 54.76 & 28 & & \\
\hline$n_{i}$ & 20 & & 8 & \\
\hline$n_{\text {total }}$ & 28 & & & \\
\hline$R_{i}$ & & 331 & & 75 \\
\hline$R_{\text {ave }}$ & & 16.55 & & 9.38 \\
\hline$U_{i}$ & 39 & & 121 & \\
\hline$U_{\min }$ & 39 & & & \\
\hline$U_{\text {crit }}$ & 34 for & $=0.0$ & for $\mathrm{p}$ & .05 \\
\hline
\end{tabular}




\section{Appendix E}

\section{Code Listing 2-1: Basic Algortihm}

This appendix contains the Matlab code listing for the basic algorithm. This simple algorithm can be applied in all cases.

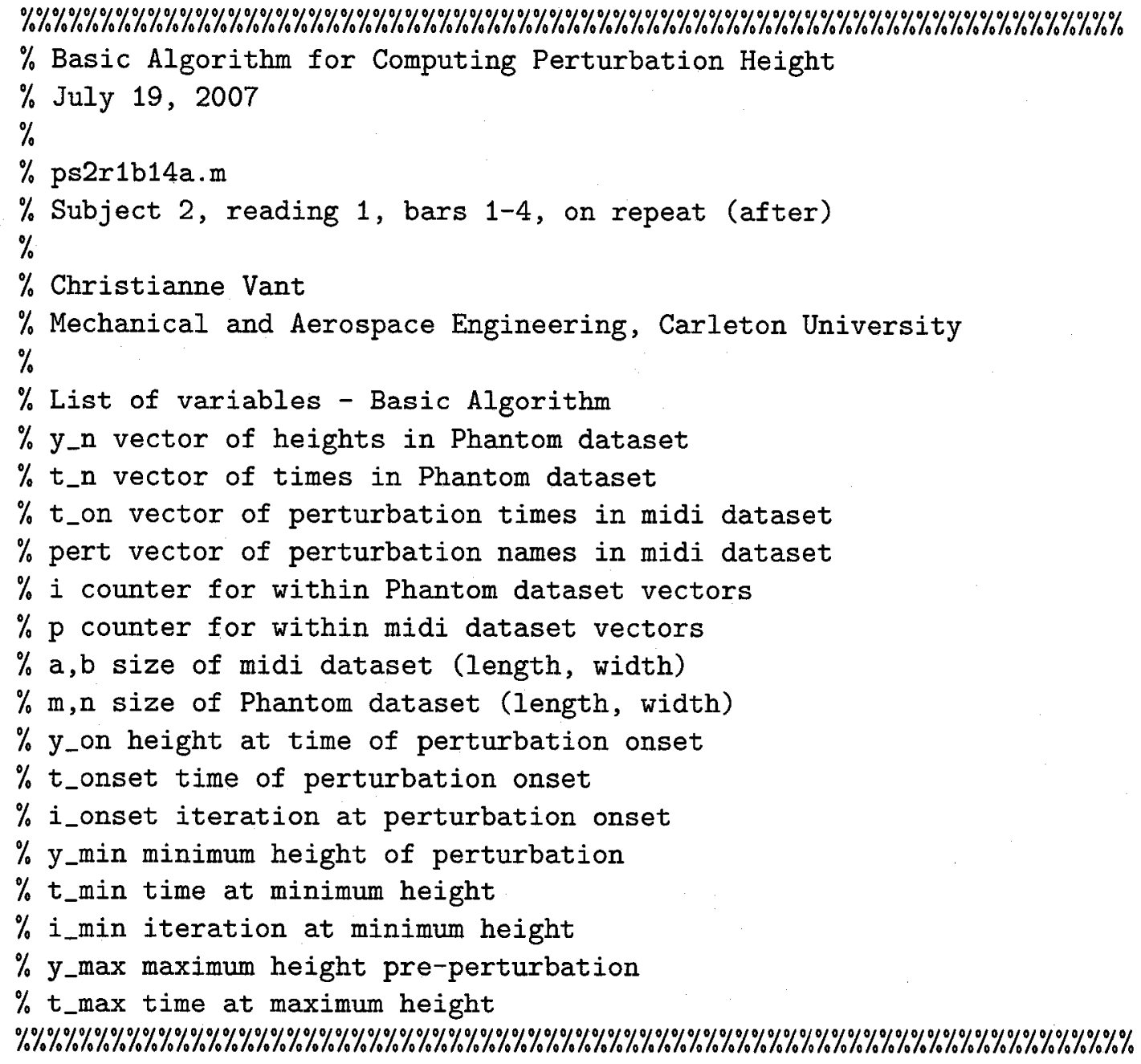


load s2r1b14ap.txt;

$\%$ two useful columns from the Phantom dataset are:

y_n $=$ s2t1aft $(:, 3) ; \%$ height

$t_{-} n=\operatorname{sit} 1$ aft $(:, 1) ; \%$ time

$\%$ other columns imported

$\% \%$ fn $=$ s2t1aft $(:, 5)$; the force

$\% \% z_{n} n=\operatorname{sit} 1$ aft $(:, 4) ;$ the $z$-position

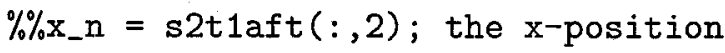

$\%$ from the midi dataset

$t_{-}$on $=\operatorname{s} 2 r 1 b 14 a p(:, 1) ; \%$ time of perturbation onset

pert $=\operatorname{s} 2 r 1 b 14 a p(:, 2) ; \%$ perturbation identifier

$\%$ initiate counters

$i=1$;

$\mathrm{p}=1$;

$\%$ find the length of the datasets

$\%$ ' $a$ ' is the length of pert and ' $m$ ' is the length of $y_{-} n$

$[\mathrm{a}, \mathrm{b}]=\operatorname{size}($ pert $)$;

$[\mathrm{m}, \mathrm{n}]=\operatorname{size}\left(\mathrm{y} \_\mathrm{n}\right)$;

$\%$ iterate through the perturbations in the midi dataset while $\mathrm{p}<\mathrm{a}+1$

$\%$ find the time and height from the Phantom dataset

$\%$ corresponding to the perturbation onset

while $1==1$

if $t_{-} n(i)==t_{-}$on $(p)$

$t_{-}$onset $=t_{-} n(i)$;

$\mathrm{y}_{-}$on $=\mathrm{y}_{-} \mathrm{n}(\mathrm{i})$;

break

elseif $t_{-} n(i)>t_{-}$on $(p)$

$t_{\text {_onset }}=t_{-} n(i)$;

$y_{-}$on $=y_{-} n(i-1)+\left(t_{-} o n(p)-t_{-} n(i-1)\right) *\left(y_{-} n(i)-y_{-} n(i-1)\right)$

$/\left(t_{-} n(i)-t_{-} n(i-1)\right)$;

break

else

$i=i+1$

continue

end

end 
$\%$ flag the iteration at the onset

i_onset $=i$;

$\%$ some of the cases required the iteration to be stepped forward

$\%$ to get over a small hump in the data

$\% i=i+5$;

$\%$ iterate through the data starting at the onset of the perturbation

$\%$ to find the minimum height, or valley, during the perturbation

while $i<m-1$

if $y_{-} n(i)<y_{-} n(i+1)$

$\mathrm{y} \_$min $_{\text {m }} \mathrm{y}_{-} \mathrm{n}(\mathrm{i})$;

$t_{-} \min =t_{-} n(i)$;

i_min $=i$;

break

end

$i=i+1$;

end

$\%$ return to the point of onset

$i=$ i_onset -1 ;

$\%$ iterate backwards through the data looking for a point greater

$\%$ than the height at the perturbation onset

$\%$ is important depending on where along the wave the perturbation began

$\%$ (i.e. before or after a valley)

while $i>0$

if $\mathrm{y}_{-} \mathrm{n}(\mathrm{i})<=\mathrm{y}_{-}$on

$i=i-1$;

else

break

end

end

$\%$ declare initial values for the maximum height, and time at that height $\mathrm{y}_{-} \max =\mathrm{y}_{-} \mathrm{n}(\mathrm{i})$;

$t_{-} \max =t_{-} n(i)$;

$\%$ iterate backwards through data to find the true maximum of that peak while $i>0$

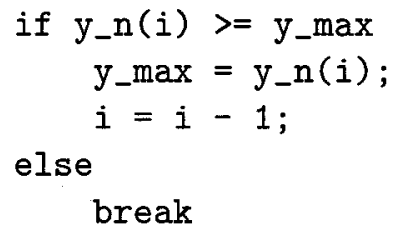




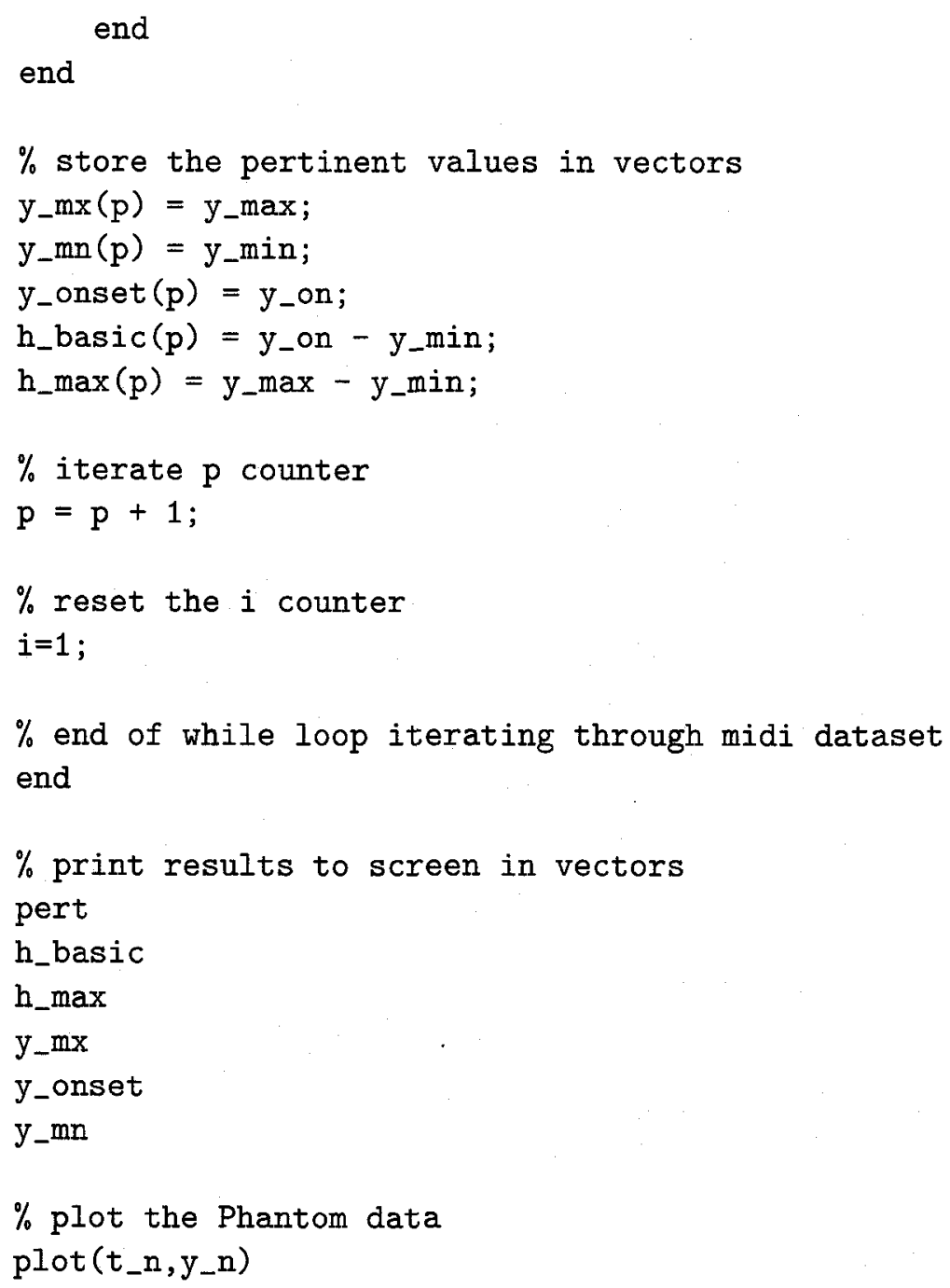




\section{Appendix F}

\section{Code Listing 2-2: Time Shift Algortihm}

This appendix contains the Matlab code listing for the time shift algorithm. The time shift algorithm requires two identical sections of music to compare, one perturbed and one unperturbed. Therefore it was only applied to perturbation 5 .

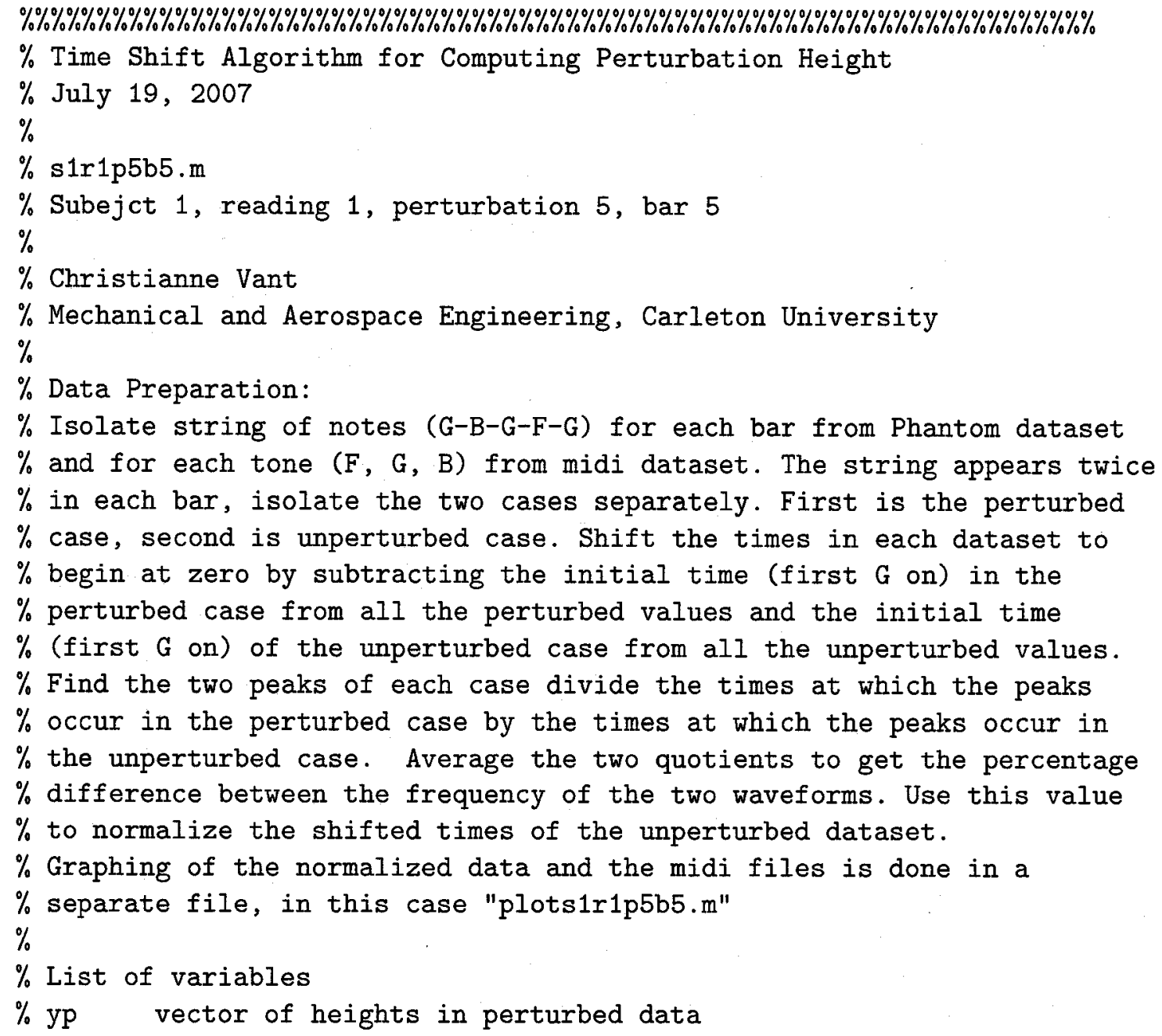


$\%$ tp vector of times in perturbed data, time shifted to begin at 0

$\% i \quad$ counter for within perturbed data

$\% \mathrm{p}, \mathrm{q}$ size of perturbed data (length, width)

$\%$ p_max maximum height for perturbed data (before perturbation onset)

$\%$ p_min minimum height for perturbed data (after perturbation onset)

$\%$ p_height difference between max and min values of the perturbed dataset

$\%$ yu vector of heights in unperturbed data

$\%$ tnorm vector of times in unperturbed data, time shifted to 0 and

$\% \quad$ normalized to match perturbed data

$\% j \quad$ counter for within unperturbed data

$\% \mathrm{u}, \mathrm{v}$ size of unperturbed data (length, width)

$\%$ u_max maximum height for unperturbed data (before perturbation onset)

$\%$ u_min minimum height for unperturbed data (after perturbation onset)

$\%$ u_height difference between max and min values of the unperturbed data

$\%$ pert_time time of the onset of the perturbation

$\%$ perturbation difference between perturbed and unperturbed heights

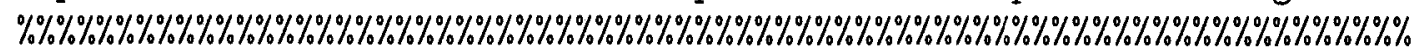

load s1r1p5b5yp.txt;

load s1r1p5b5yu.txt;

$\%$ two useful columns from the perturbed dataset are:

yp $=\operatorname{sir} 1 \mathrm{p} 5 \mathrm{~b} 5 \mathrm{yp}(:, 4) ; \%$ the height

tp $=\operatorname{s1r} 1 \mathrm{p} 5 \mathrm{~b} 5 \mathrm{yp}(:, 2) ; \%$ the time-shifted time

$\%$ other columns are:

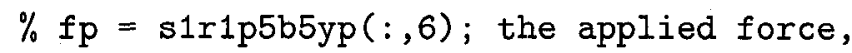

$\% \mathrm{zp}=\operatorname{sir} 1 \mathrm{p} 5 \mathrm{~b} 5 \mathrm{yp}(:, 5)$; the $\mathrm{z}$ position,

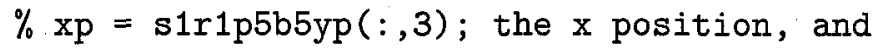

$\%$ t1 $=\operatorname{sir} 1 \mathrm{p} 5 \mathrm{b5yp}(:, 1) ;$ the original time

$\%$ two useful columns from the unperturbed dataset are:

$\mathrm{yu}=\operatorname{s1r} 1 \mathrm{p} 5 \mathrm{~b} 5 \mathrm{yu}(:, 5) ; \%$ the height

tnorm $=\operatorname{s1r} 1$ p5b5yu $(:, 3) ; \%$ the time-shifted and normalized time

$\%$ other columns are:

$\% \mathrm{fu}=\mathrm{s} 1 \mathrm{r} 1 \mathrm{p} 5 \mathrm{~b} 5 \mathrm{yu}(:, 7)$; the applied force,

$\% \mathrm{zu}=\operatorname{s1r} 1 \mathrm{p} 5 \mathrm{~b} 5 \mathrm{yu}(:, 6) ;$ the $\mathrm{z}$ position,

$\% \mathrm{xu}=\operatorname{s1r} 1 \mathrm{p} 5 \mathrm{~b} 5 \mathrm{yu}(:, 4) ;$ the $\mathrm{x}$ position,

$\%$ tu $=\operatorname{sir} 1 \mathrm{p} 5 \mathrm{~b} 5 \mathrm{yu}(:, 2) ;$ the time-shifted time, and

$\%$ t2 $=\operatorname{sir} 1 \mathrm{p} 5 \mathrm{~b} 5 \mathrm{yu}(:, 1)$; the original time

$\%$ initiate counters

$i=1$;

$j=1$; 


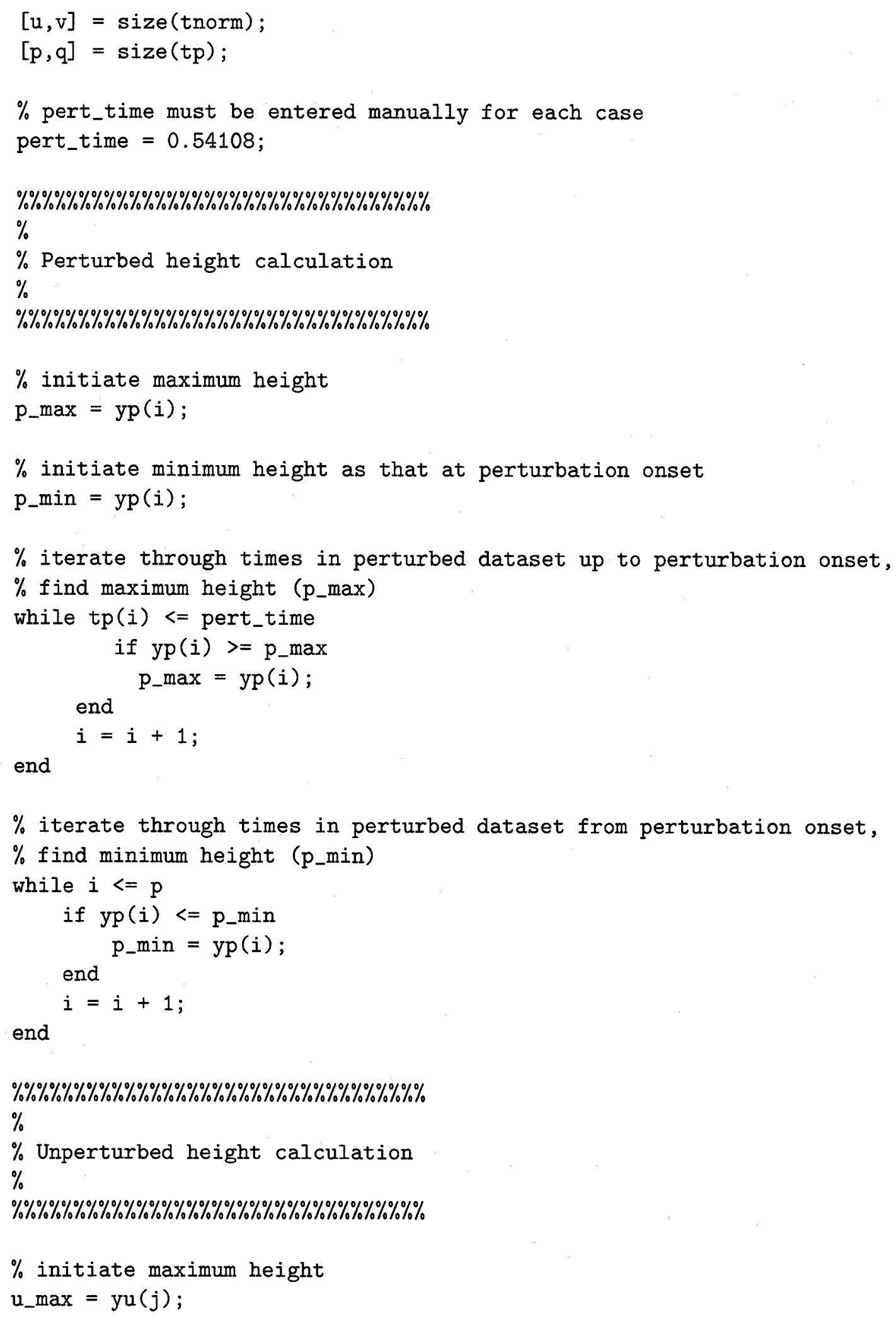


$\%$ initiate minimum height as that at perturbation onset

$u_{-} \min =y u(j)$;

$\%$ iterate through times in unperturbed dataset up to perturbation onset, $\%$ find maximum height (u_max)

while $\operatorname{tnorm}(j)<=$ pert_time

if $\mathrm{yu}(j)>=u_{-} \max$

$u_{-} \max =\mathrm{yu}(\mathrm{j})$;

end

end

$j=j+1$

$\%$ iterate through times in unperturbed dataset from perturbation onset $\%$ onward, find minimum height (u_min)

while $\mathrm{j}<\mathrm{u}$

if $\mathrm{yu}(j)<=u_{-} \min$

$u_{-} \min =\operatorname{yu}(j)$;

end

$j=j+1$

end

$\%$ print results to screen

p_min

p_max

p_height $=$ P_max - p_min

u_min

u_max

u_height $=u_{-} \max -u_{-} \min$

perturbation = p_height $-u_{-}$height 


\section{Appendix G}

\section{Code Listing 2-3: Symmetry Algortihm}

This appendix contains the Matlab code listing for the symmetry algorithm. Because the algorithm assumes data in a waveform, this algorithm was only applied to subject 1 .

This code listing includes sections of code that are required for analysis of perturbation 6 , but not for perturbation 5 . This difference is due to the location of the perturbed note in the groupings; for perturbation 5 the note is in the middle of the grouping while for perturbation 6 it is the first note in the grouping.

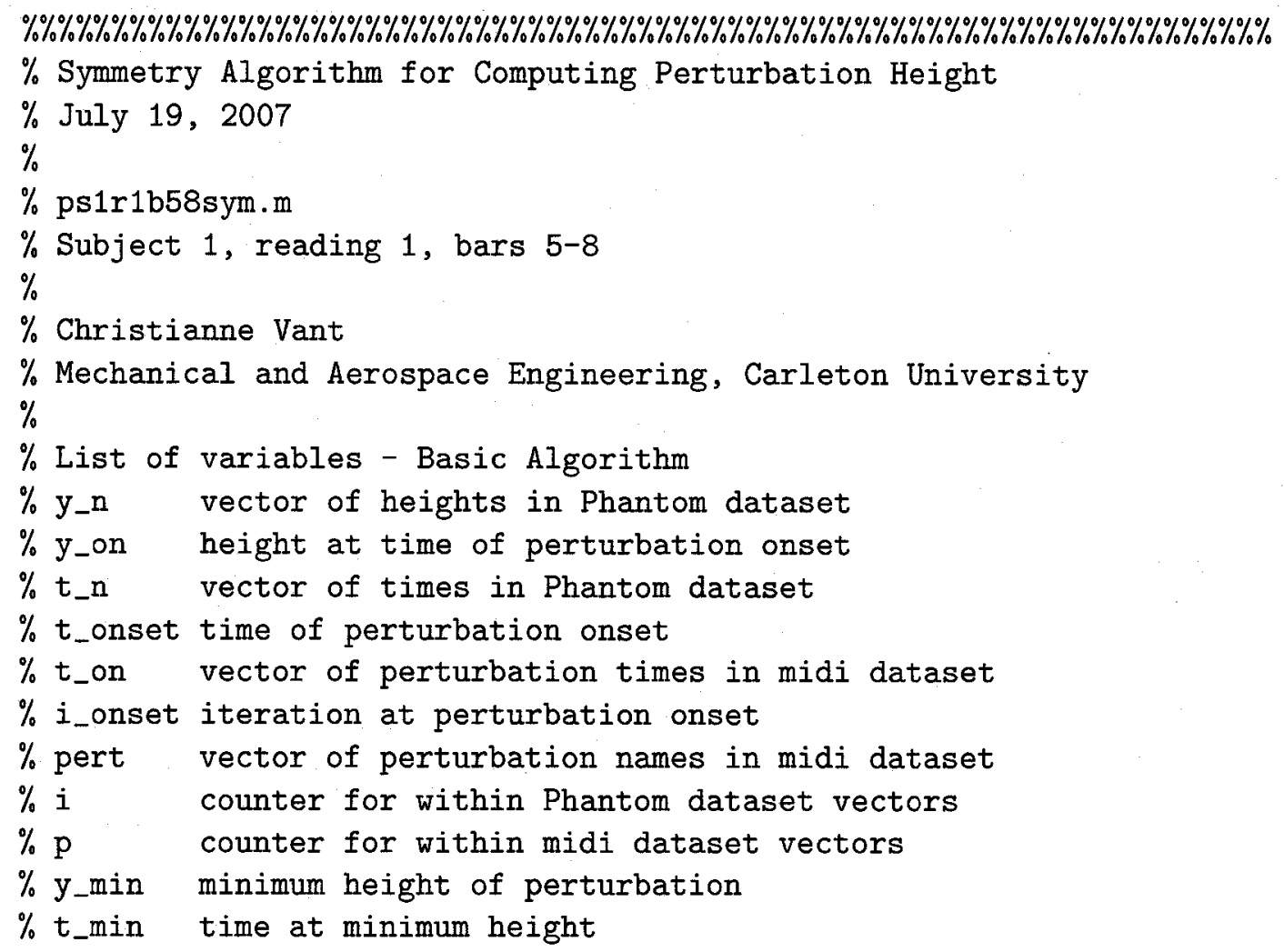




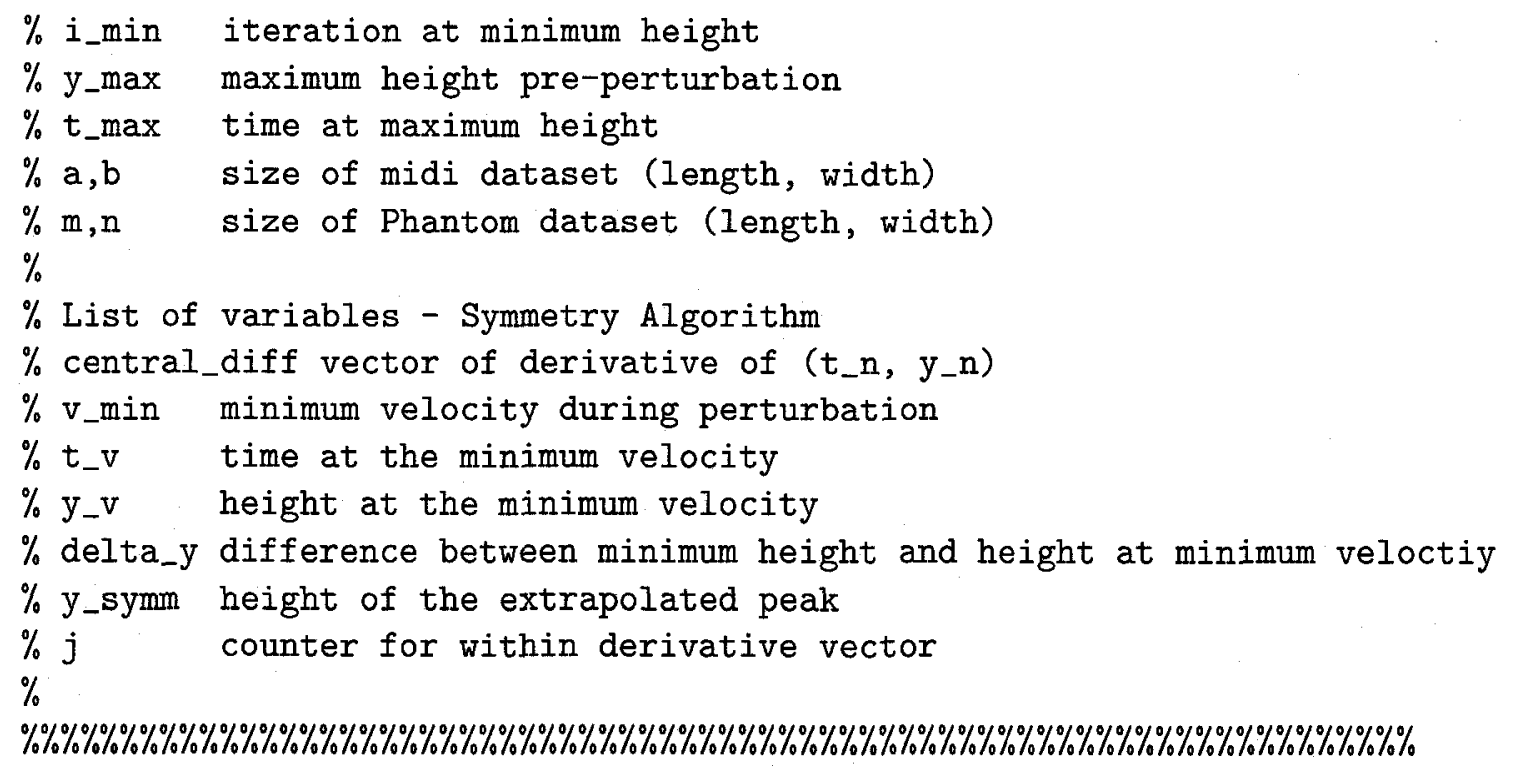

load s1t1bef.txt;

load s1r1b58p.txt;

$\%$ two useful columns from the Phantom dataset are:

$\mathrm{y}_{-} \mathrm{n}=$ s2t1aft $(:, 3) ; \%$ the height

$t_{-} n=\operatorname{sit} 1$ aft $(:, 1) ; \%$ the time

$\%$ other columns imported

$\%$ f_n $=$ s2t1aft $(:, 5) ; \quad$ the force

$\% \% z_{n} n=\operatorname{sit} 1$ aft $(:, 4) ; \quad$ the $z$-position

$\% \% x_{-} n=s 2 t 1 a f t(:, 2) ; \quad$ the $x$-position

$\%$ from the midi dataset:

$t_{-}$on $=\operatorname{s1r} 1 b 58 p(:, 1) ; \%$ the time of the perturbation onset

pert $=\operatorname{sir} 1 \mathrm{~b} 58 \mathrm{p}(:, 2) ; \%$ the perturbation identifier

$\%$ initiate counters

$i=1$;

$j=2$;

$\mathrm{p}=1$

$[\mathrm{a}, \mathrm{b}]=\operatorname{size}($ pert) $; \% \mathrm{a}$ is the length of the midi dataset

$[\mathrm{m}, \mathrm{n}]=\operatorname{size}\left(\mathrm{y}_{-} \mathrm{n}\right) ; \% \mathrm{~m}$ is the length of the Phantom dataset

$\%$ create a matrix of zeros to hold the derivative calculation central_diff $=\operatorname{zeros}(\mathrm{m}, 1)$;

$\%$ calculate the derivative of the height data using the 
$\%$ central difference approximation

while $\mathrm{j}<\mathrm{m}-1$

central_diff $(j, 1)=\left(y_{-} n(j+1)-y_{-} n(j-1)\right) /\left(t_{-} n(j+1)-t_{-} n(j-1)\right)$;

$j=j+1$;

end

\%\%\%\%\%\%\%\%\%\%\%\%\%\%\%\%\%\%\%\%\%\%\%\%\%\%\%\%\%\%\%\%\%\%\%\%\%\%\%\%\%\%\%\%\%\%\%\%\%\%\%\%\%\%\%\%

$\%$

$\%$ Basic Algorithm

$\%$

\%\%\%\%\%\%\%\%\%\%\%\%\%\%\%\%\%\%\%\%\%\%\%\%\%\%\%\%\%\%\%\%\%\%\%\%\%\%\%\%\%\%\%\%\%\%\%\%\%\%\%\%\%\%\%\%\%

$\%$ iterate through the perturbations in the midi dataset

while $\mathrm{p}<\mathrm{a}+1$

$\%$ find the time and height from the Phantom dataset corresponding to $\%$ the perturbation onset

while $1==1$

if $t_{-} n(i)==t_{-}$on $(p)$

$t_{-}$onset $=t_{-} n(i)$;

$y_{-}$on $=y_{-} n(i)$;

break

elseif $t_{-} n(i)>t_{-} o n(p)$

$t_{-}$onset $=t_{-}$on $(p)$;

$y_{-} o n=y_{-} n(i-1)+\left(t_{-} o n(p)-t_{-} n(i-1)\right) *\left(y_{-} n(i)-y_{-} n(i-1)\right)$

$/\left(t_{-} n(i)-t_{-} n(i-1)\right)$;

break

else

$i=i+1$

continue

end

end

$\%$ flag the iteration at the onset

i_onset $=i$;

$\%$ some of the cases required the iteration to be stepped forward

$\%$ to get over a small hump in the data

$\% i=i+5$;

$\%$ iterate through the data starting at the onset of the perturbation $\%$ to find the minimum height (y_min),

$\%$ or valley, during the perturbation

while $i<m-1$

if $y_{-} n(i)<y_{-} n(i+1)$ 


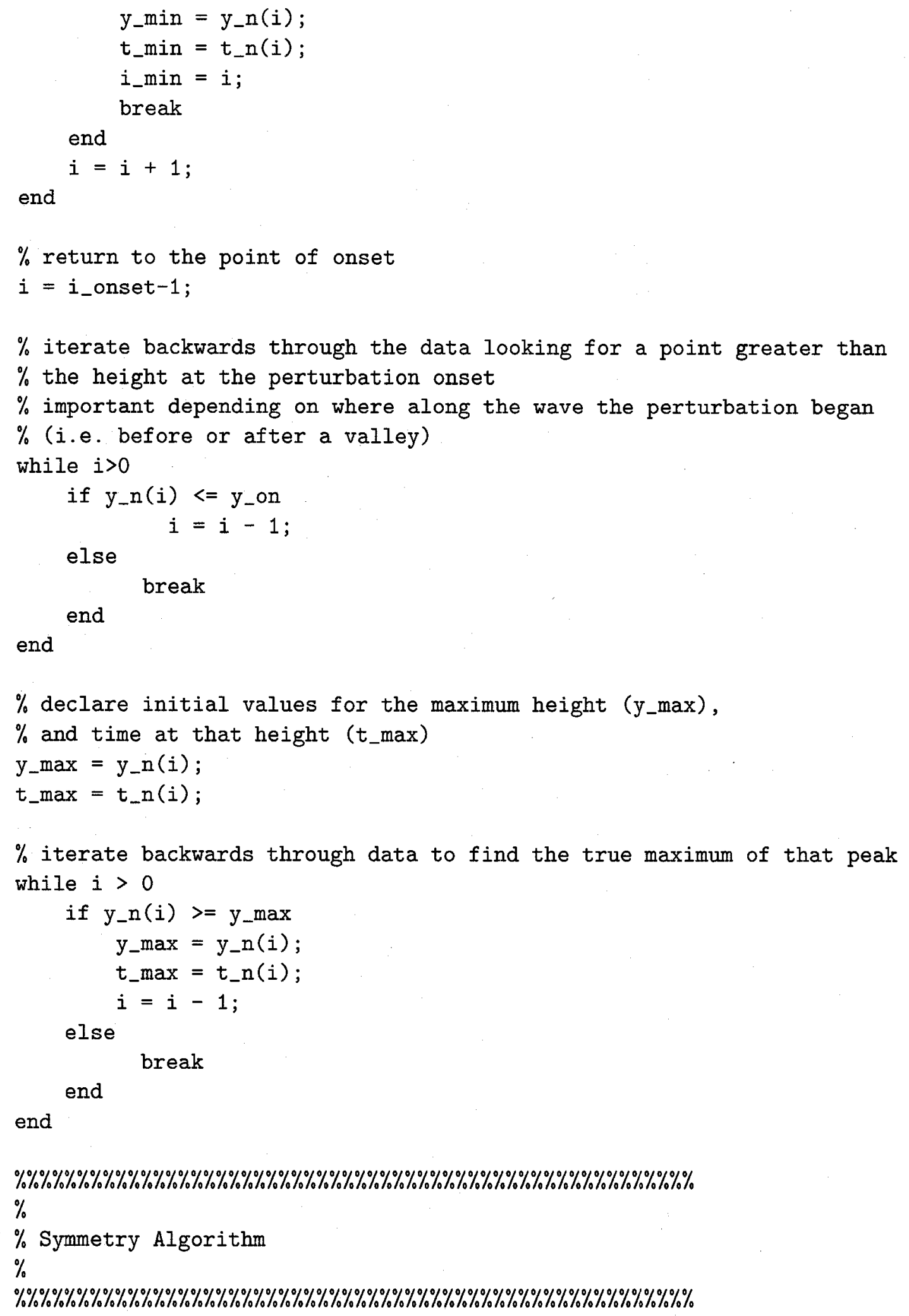


$\%$ return to the point of onset

$i=$ i_onset;

$\%$ initiate values for the minimum derivative

v_min = central_diff(i);

$t_{-} v=t_{-} n(i)$;

$\mathrm{y}_{-} \mathrm{v}=\mathrm{y}_{-} \mathrm{n}(\mathrm{i})$;

$\%$ in some cases, perturbation 6 required modification because the

$\%$ perturbation onset occurred at the valley of a wave, and the

$\%$ appropriate minimum was before the onset, not after it

$\%$ if $p<5$

$\%$ iterate through the derivative to find the minimum

$\%$ (i.e. the steepest downward slope)

while $i<$ i_min

if central_diff $(i)<v_{-} \min$

$v_{-} \min =$ central_diff $(i)$;

$t_{-} v=t_{-} n(i)$;

$y_{-} v=y_{-} n(i)$;

end

$i=i+1$;

end

$\%$ Continuation of modifications for perturbation 6 ...

$\%$ iterate backwards through the derivative to find the minimum

$\%$ (i.e. the steepest downward slope)

$\%$ else

$\%$ while $t_{-} n(i)>t_{-} \max$

$\% \quad$ if central_diff $(i)<v_{-} \min$

$\% \quad$ v_min $=$ central_diff(i);

$\% \quad t_{-} v=t_{-} n(i)$;

$\% \quad y_{-} v=y_{-} n(i)$;

$\% \quad$ end

$\% \quad i=i-1$;

$\% \quad$ end

$\%$ end

$\%$ calculate the height (y_symm) of the extrapolated peak,

$\%$ equal to $\mathrm{y}_{-} \mathrm{v}+\mathrm{y}$

$\%$ where $\mathrm{y}$ is the height at minimum velocity minus the minimum height

delta_y $=y_{-} v-y_{-} \min$;

$y_{-}$symm $=y_{-} v+\operatorname{delta} y$;

$\%$ store the pertinent values in vectors 


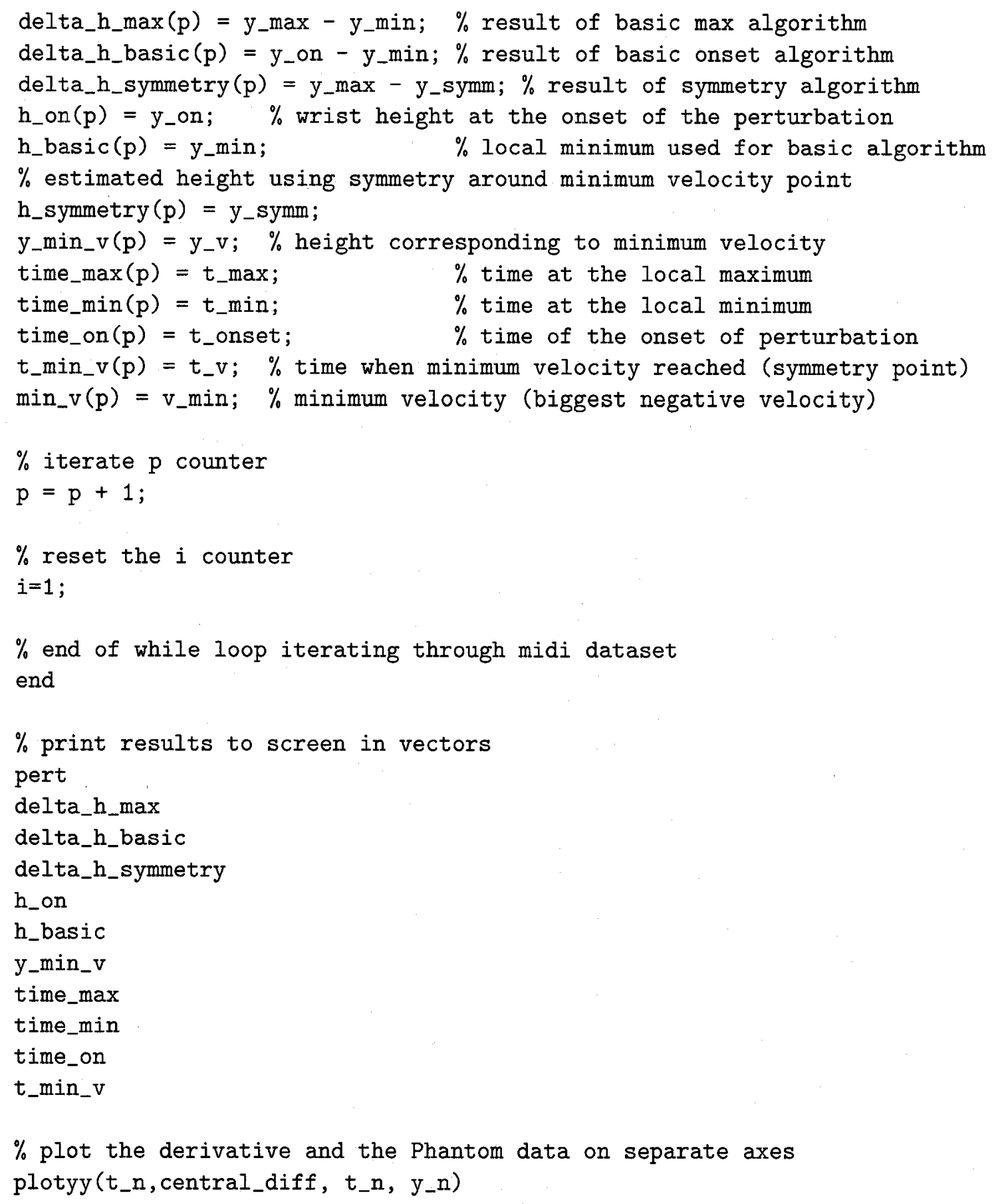

